PUB-5399

UC-414

\title{
Exclusive Study of Nuclear Collisions at the AGS
}

P. Brady, D. Cebra, M. Partlan, and J. Romero

University of California

Davis, CA 95616

S. Albergo, Z. Caccia, S. Costa, A. Insolia, and R. Potenza

INFN Catania, Italy

M. Justice, D. Keane, and D. Weerasundara

Kent State University

Kent, OH 44242

W. Gong, L. Heilbronn, J. Miller, D. Olson, G. Rai, H.G. Rittler,

J. Rusmussen, $\mathrm{H}$. Weiman, and C. Zeitlin

Lawrence Berkeley Laboratory, Berkeley, CA 94720

G. Peilert

Lawrence Livermore National Laboraiury

Livermore, CA 94550

A. Hirsch, E. Hjort, N. Porile, R. Scharenberg, and B. Shrivastava

Purdue University, West Lafayette, IN 47907

A.D. Chacon and K. Wolf

Texas A\&M University, College Station, TX 77843

August 1993

This work was supported by the Director, Office of Energy Research, Division of Nuclear Physics of the Office of High Energy and Nuclear Physics, of the U.S. Department of Energy under Contract No. DE-AC03$76 S F 00098$. 


\section{AGS PROPOSAL}

TITLE:

FROM:

SPOKESPERSON:

BEAMLINE:

BEAM:
Exclusive Study of Nuclear Collisions at the AGS

P. Brady, D. Cebra, M. Partlan, J. Romero

University oi California, Davis, CA 95616

S. Albergo, Z. Caccia, S. Costa, A. Insolia, R. Potenza, J. Romanski, G.V. Russo, C. Tuve INFN Catania, Italy

M. Justice, D. Keane, D. Weerasundara

Kent State University, Kent, OH 44242

W. Gong, L. Heilbronn, J. Miller, D. Olson, G. Raj

H. G. Ritter, J. Rasmussen, H. W'ieman, C. Zeitlin

Lawrence Berkeley Laboratory, Berkeley, CA 94720

G. Peilert

Lawrence Livermore National Laborarory, Livermor : CA 94550

A. Hirsch, E. Hjort, N. Porile, R. Scharenberg, B. Shrivastava Purdue University, West Lafayette, IN 47907

A. D. Chacun, K. Wolf

Texas A \& M University, College Station, TX 77843

S. Mordechai

University of Texas, Austin, TX 78712

G. Rai

Prefer the A1 beamline with MPS magnet or alternatively the $\mathrm{B} 1$ or $\mathrm{C5}$ lines with suitable magnet. Location not yet decided.

Initially Au primary beam with intensity of $1000 / \mathrm{sec}$ and $1 \mathrm{sec}$ flat top. A higher beam flux with a longer flat top is acceptable. The highest beam energy at which the AGS will operate reliably is requested. Subsequent experiments will require lower beam energies - refer to the run schedule.

EOS TPC in the MPS or other suitable magnet, MUSIC 
detector, TOF Wal, MUFFINS neutron spectrometer, Beam vectoring and trigger detectors. All detectors will be shipped from LBL.

OLDF:

Not required.

TIME REQUESTED:

CONTACT:
Au Beam at highest energy 336 hours and 1352 hours at the lower beam energies.

Gulshan Rai

Lawsence Berkeley Laboratory, Nuclear Science Division, MS50D-121,

1 Cyclotron Road, Berkeley, CA 94720

Tel: $510-486-7125$

Fax: $510-486-4818$

Email: RAI@LBL.bitnet

This work was supported by the Director, Office of Energy Research,

Division of Nuclear Physics of the Office of High Energy and

Nuclear Physics of the U.S. Department of Energy under Contract DE-AC03-76SF00098 


\title{
Exclusive Study of Nuclear Collisions at the AGS
}

\author{
August 19, 1993
}

\begin{abstract}
We propose to carry out a systematic and exclusive measurement of the energy and mass dependence of particle production, correlations and collective effects in Au+ $\mathrm{Au}$ collisions. We wish to determine the highest compression achievable in nuclear matter and to study its properties. We shall search for evidence for an exotic Equation of -State, that is, new physics such as Resonance Matter, Exotica, and QGP. We are also interested in signatures of critical phenomens in dilute nuclear matter.

We propose to measure the four-momentum of light mass particles $\left(\pi^{ \pm}, K_{\ddagger}^{\circ}, K^{ \pm}, \Lambda\right.$, $\mathrm{n}, \mathrm{p}, \mathrm{d},{ }^{3} \mathrm{He},{ }^{4} \mathrm{He},{ }^{6} \mathrm{He}$, and the isotopes of $\mathrm{Li}$ and $\mathrm{Be}$ ), projectile fragments from $Z=6$ to $Z=79$, and anti-proton production. The majority of the data will be acquired, on an event by event basis, from a state-of-the-art Time Projection Chamber (EOS TPC) built and used at LBL by the EOS collaboration. The TPC provides continuous tracking, almost $4 \pi$ acceptance and particle ideatification for the light mass particles.
\end{abstract}


Exclusive Study of Nuclear Collisions at the AGS

P. Brady, D. Cebra, M. Partlan, J. Romero

University of California, Davis, CA 95616

S. Albergo, Z. Caccia, S. Costa, A. Insolia, R. Potenza

J. Romanski, G.V. Russo, C. Tuve

INFN Catanja, Italy

M. Justice, D. Keane, D. Weerasundara

Kent State University, Kent, $\mathrm{OH} 44242$

W. Gong, J. Miller, L. Heilbronn, D. Olson, G. Rai

H. G. Ritter, J. Rasmussen, H. Wieman, C. Zeitlin

Lawrence Berkeley Laboratory, Berkeley, CA 94720

G. Peilert

Lawrence Livermore National Laborarory, Livermore, CA 94550

A. Hirsch, E. Hjort, N. Porile, R. Scharenberg, B. Shrivastava Purdue University, West Lafayette, IN 47907

A. D. Chacon, K. Wolf

Texas A \& M University, College Station, TX 77843

S. Mordechai

University of Texas, Austin, TX 78712 


\section{Contents}

$\begin{array}{lll}1 \text { Introduction } & \theta\end{array}$

2 The Physics of Hot Dense Nuclear Matter 12

2.1 Nuclear Matter and the Equation of State . . . . . . . . . . . . . . 12

2.2 Resonance Matter ... . . . . . . . . . . . . . . . . . 14

2.3 Collective Phenomena and their Importance . . . . . . . . . . . 16

2.4 Measuring Flow in Nuclear Matter ................. . . 16

2.5 Flow as a Signature of Quark-Gluon Plasma . . . . . . . . . . . . . 23

3 Proposed Measurements 24

3.1 Collective Effects . . . . . . . . . . . . . . . . . . . . 24

3.2 Stopping . . . . . . . . . . . . . . . . . . . . 24

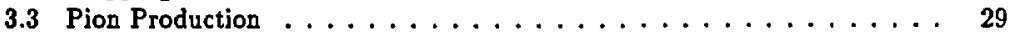

3.4 Strange Particle Production ..................... 31

3.5 Antiproton Production . . . . . . . . . . . . . . . . . 32

3.6. Intensity Interferometry . . . . . . . . . . . . . . . 34

3.7 Composite Fragment Production ... . . . . . . . . . . . . 36

4 Physics of Dilute Matter $\quad 38$

4.1 A General Overview of Multifragmentation . . . . . . . . . . . . . 38

4.2 Niuclear Fragmentation as a Critical Phenomenon . . . . . . . . . . . 39

5 Experimental Configuration 46

5.1 The EOS Time Projection Chamber . . . . . . . . . . . . . 48

5.1 .1 Detector Description . . . . . . . . . . . . . . . . 48

5.1 .2 Laser Calibration System .................. 52

5.1.3 Event reconstruction using real data ............... 52

5.1 .4 Particle Identification and $d E / d x$ Resolution .......... 55

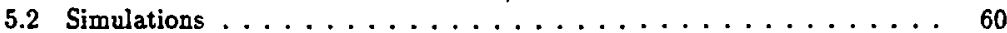

5.2.1 TPC Space Point Reconstruction ................. 60

5.2 .2 Track Reconstruction Efficiency . . . . . . . . . . . . . 61

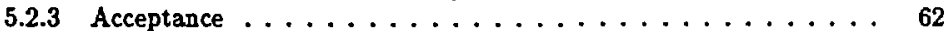

5.2 .4 Transverse Momentum Spectra ................ 67

5.3 The Multiple Sampling Ionization Chamber MUSIC . . . . . . . . . . 67

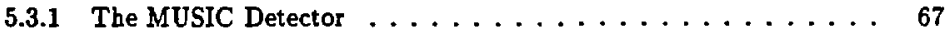

5.3 .2 Performance ....................... 70

5.4 The Time-of-Flight Wall .................... 70

5.5 The Neutron Spectrometer MUFFINS .............. 73

$5.5 .1 \mathrm{n}-\gamma$ Discrimination . . . . . . . . . . . . . . 75

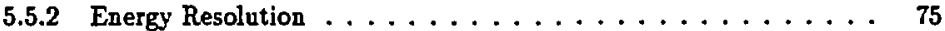

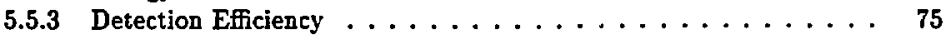

5.5.4 Geometric and Total Acceptance .............. 77

5.6 Summary of Capability . . . . . . . . . . . . . . 78

5.7 Beamline Detectors and Trigger . . . . . . . . . . . . 78 
5.8 Advanced Detector Technology . . . . . . . . . . . . . 81

5.8 .1 The Micro-strip Readout TPC . . . . . . . . . . 81

5.8 .2 Silicon Drift Detectors . . . . . . . . . . . . . . 84

6 AGS Requirements and Logistics 85

6.1 Readiness . . . . . . . . . . . . . . . . 85

6.2 Responsibilities ... . . . . . . . . . . . . . . . 85

6.3 Beam Line and Experimental Area . . . . . . . . . . . . 86

6.4 Beam Energies, Particles and Time Request . . . . . . . . . 87

6.5 Schedule . . . . . . . . . . . . . . . . . . . . . 89 


\section{List of Figures}

1 Phase diagram of nuclear matter. . . . . . . . . . . . . .

2 The nuclear compressional energy as a function of density for two different values of the compressibility factor $K \ldots \ldots \ldots \ldots \ldots \ldots$

3 The exotic EOS including delta resonances at $T=0 \mathrm{MeV}$ for different

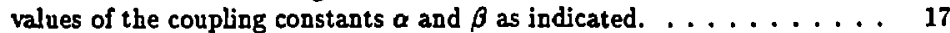

Schematic illustration of a nucleus-nucleus collision.

Transverse momentum analysis. . . . . . . . . . . . . . .

6 Flow parameters for the EOS data talen at the Bevalac for $\mathrm{Au}+\mathrm{Au}$. (These data are preliminary.) . . . . . . . . . . . . . . Flow excitation function for protons at the Bevalac. (These data are preliminary.) . . . . . . . . . . . . . . . . .

8 Transverse momenta for a RQMD simulation of $10.7 \mathrm{~A} \mathrm{GeV}$ (beam energy)

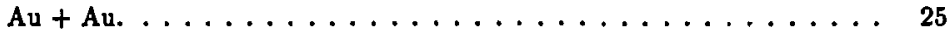
RQMD predictions for proton and deuteron flow for $10.7 \mathrm{~A} \mathrm{GeV} \mathrm{Au}+\mathrm{Au}$.

10 Flow for hard and soft equations of state at $10.7 \mathrm{~A} \mathrm{GeV}$ in the RQMD model. 27

11. VUU calculation for the excitation function of the directed transverse flow $p_{x}$. The upper curve was obtained using the hard EOS, while for the lower curve the exotic EOS was used. . . . . . . . . . . . . . Schematic illustration of pion absorption and re-scattering by spectator matter.

13 Reconstructed $d E / d x$ vs $p / Z$ of strange primaries and their $p$ and $\pi$ decay products from $20010.7 \mathrm{~A} \mathrm{GeV} \mathrm{Au}+\mathrm{Au}$ ARC events. A cut requiring at least 20 hits in the TPC has been applied. . . . . . . . . 33 Anti-proton production at $10.7 \mathrm{~A} \mathrm{GeV}$ as predicted by RQMD . . . . . Fragment yield distribution for $10.7 \mathrm{~A} \mathrm{GeV} \mathrm{Au}+\mathrm{Au} . \ldots \ldots \ldots 37$ Schematic of mass spectra for $T<T_{c}, T \sim T_{c}$, and $T>T_{c}$. . . . . $\ln \left(M_{3}\right)$ versus $\ln \left(M_{2}\right)$ for a 5000 event simulation for a 108 nucleon system.

18 Percolation simulation predictions of $\ln \left(M_{2}\right)$ versus $n$ for systems of different sizes. . . . . . . . . . . . . . . . . 43

19 Preliminary data analysis of $1.0 \mathrm{~A} \mathrm{GeV} \mathrm{Au}+\mathrm{C}$ EOS dita for $\log \left(M_{3}\right)$ versus $\log \left(M_{2}\right) \ldots \ldots \ldots \ldots \ldots \ldots \ldots \ldots \ldots \ldots \ldots$

20 Average size of the largest fragment and fluctuations. . . . . . . . . 45

21 Proposed set up for the EOS experiment at the AGS (perspective view). . 47

22 The EOS TPC. . . . . . . . . . . . . . . . . .

The pad plane of the EOS TPC. . . . . . . . . . .

24 Perspective view of a $\mathrm{Au}+\mathrm{Au}$ event at $1.0 \mathrm{~A} \mathrm{GeV}$ beam energy. . . . . .

25 Reconstructed $\mathrm{Au}+\mathrm{Au}$ event projected onto the pad plane. . . . . .

26 Scatter plot of $d E / d x$ vs rigidity for particles in the Bevalac experiment

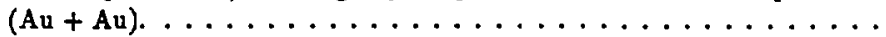

27 Scatter plot of $d E / d x$ vs rigidity for particles in the Bevalac experiment

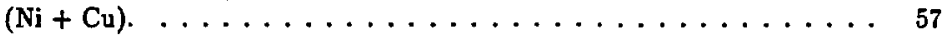

28 Scatter plot of $d E / d x$ vs $p / Z$ for $8.9 \mathrm{~A} \mathrm{GeV} / \mathrm{c} \mathrm{Au}+$ Au ARC simulations. 58

29 Scatter plot of $d E / d x$ vs $p / Z$ for $4.8 \mathrm{~A} \mathrm{GeV} / \mathrm{c} \mathrm{Au}+$ Au ARC simulations. 
30 Transverse momentum vs rapidity for protons and pions for $6 \mathrm{~A} \mathrm{GeV} \mathrm{Au}$ + Au RQMD simulations. ..................... 63

31 Transverse momentum vs rapidity for accepted protons and pions for $6 \mathrm{~A} \mathrm{GeV}$ $A u+A u$ RQMD simulations. . . . . . . . . . . . . . . . 64

32 Transverse momentum vs rapidity for protons and pions for $10 \mathrm{~A} \mathrm{GeV} \mathrm{Au}$ + Au RQMD simulations. . . . . . . . . . . . . . . . . 65

33 Transverse momentum vs rapidity for accepted protons and pions for $10 \mathrm{~A} \mathrm{GeV}$ $\mathrm{Au}+\mathrm{Au}$ RQMD simulations. . . . . . . . . . . . . . . 66

34 Transverse momentum spectra for mid-rapidity protons (generated, accepted, and found) for a $6 \mathrm{~A} \mathrm{GeV} \mathrm{Au} \mathrm{+} \mathrm{Av} \mathrm{RQMD} \mathrm{simulation.} \mathrm{.} \mathrm{.} \mathrm{.} \mathrm{.} \mathrm{.} 68$

35 The MUSIC detector. . . . . . . . . . . . . . . . . . 69

36 A track reconstructed in the MUSIC detector. Data from the Bevalac run. 71

37 A charge spectrum as measured by MUSIC for $1.0 \mathrm{~A} \mathrm{GeV} \mathrm{Au} \mathrm{+} \mathrm{C} \mathrm{data}$ taken at the Betalac. . . . . . . . . . . . . . . . . 72

38 A front view of a MUFFIN disk and the PMTs. . . . . . . . . . . . 74

39 Energy spectrum measured with MUFFINS for the ${ }^{10} \mathrm{Ca}+\mathrm{H}$ reaction at $0.4 \mathrm{~A} \mathrm{GeV} . \ldots \ldots \ldots \ldots \ldots$

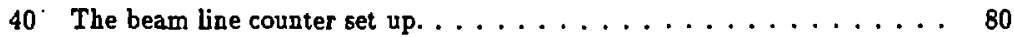

41 A Micro-strip gas counter. . . . . . . . . . . . . . . . . . . 82

42 Possible design of a Micro-strip TPC. . . . . . . . . . . . 83 


\section{Introduction}

We propose to carry out a systematic and exclusive measurement of the energy and mass dependence of particle production, correlations, and collective effects in high energy heavy ion collisions at the AGS. Our goal is to find the beam energy at which the maximum baryon density is achieved and to study the properties of highly compressed, dense nuclear matter. Recent experimental data on nuclear matter flow obtained at the Bevalac, along with theoretical calculations, indicate the highest compression in nuclear unatter is likely to occur in the 2-10 A GeV energy range.

We also propose to carry out a high statistics study of nuclear multifragmentation in inverse linematics Au + p collisions. The emergence of critical phenomena (liquid-vapour phase transition) will be examined by varying the energy deposited into the Au projectile nucleus.

The four-momentum of light mass particles $\left(\pi^{ \pm}, K_{E}^{\circ}, K^{ \pm}, \Lambda, \Xi^{-}, \Omega^{-}, n, p, d, \bar{p}\right)$ and composite fragments ( $Z=2$ to $Z=79$ ) will be measured on an event-by-event basis over a substantial fraction of $4 \pi$. The bulk of the data will be acquired from a state of the art Time Projection Chamber (TPC) built and used at LBL by the EOS collaboration. Projectile fragments will be identified in a multiple ionization sampling device called MUSIC and a time of flight (TOF) wall. Neutron measurements will be made using a scintillator based detector called MUFFINS. This experimental arrangement provides a very powerful facility to study the complete event in fine detail and simultaneously measure many observables.

The acceleration of Au beams at the AGS in 1992 has opened a new frontier of nuclear physics, namely the investigation of nuclear matter and its Equation of State (EOS ${ }^{1}$ at high energy and baryon density. The specific research goals of our experiment are to:

1. Search for evidence of collective effects such as nuclear matter flow. Collective flow is a measure of the compression achieved in high energy nucleus-nucleus collisions. Its existence at Bevalac energies has been an established fact for many years. Strong evidence from both theory and experiment indicate that even larger collective effects may be present at beam energies above $2 \mathrm{~A} \mathrm{GeV}$

2. Study the nuclear matter EOS which depends on baryon density and temperature. The density dependence is mapped by varying the beam energy and therefore the amount of nuclear compression.

3. Look for evidence of an exotic EOS, that is new physics such as Resonance Matter and/or QGP.

4. Determine the degree of nuclear stopping and thermalization.

5. Understand the collision dynamics and particle production mechanisms.

6. Study the energy dependence of critical phenomena in dilute nuclear matter.

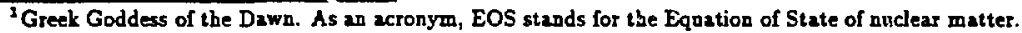
The connection betwenn the two is suggested by the book "EOS or, The Wider Aspects of Cosmogony", by J. H. Jeans, F.R.S., Kegan Paul, Trench, Tribner \& Co. Ltd., London, 1931, which explores the relationship between the strueture of matter and the creation of the universe.
} 
In order to achieve these goals me shall:

1. Measure $\pi^{ \pm}, \mathrm{h}^{ \pm}, \mathrm{K}_{i}^{\circ}, \mathrm{p}^{ \pm}, \Lambda^{\circ}, \Xi^{-}$, and $\Omega^{-}$over a wide kinematical domain, acquiring as much correlated information as possible on individual events.

2. Measure collective phenomena and nuclear matter flow: side splash, bounceoff, and squeeze-out for $p, d, \Lambda,{ }^{3} \mathrm{He},{ }^{4} \mathrm{He}$; and evidence for pion rescattering and $\tilde{\mathrm{p}}$ annihilation in spectator matter.

3. Measure strange particle yields and inclusive spectra. Determine the ratios of doubly and triply strange particles to singly strange particles: $\frac{\bar{z}}{\Lambda}, \frac{\Omega}{\Lambda}$.

4. Study Hanbury-Brown-Twiss (HBT) correlations in $\pi, \mathrm{K}^{+}, \mathrm{K}_{\mathrm{a}}$, and proton pairs.

5. Obtain $y, p_{\perp}$ distributions. Study stopping and thermalization as a function of beam energy, hence baryon density.

6. Measure composite particle yields and obtain the entropy. Study coalescence.

7. Measure heavy fragments in normal and inverse kinematics. Look for critical phenomena in dilute nuclear matter.

The EOS Time Projection Chamber will be used as our central tracking detector. It will provide almost $4 \pi$ acceptance, momentum measurement, and particle identification. It is compatible with $\mathrm{Au}+\mathrm{Au}$ collisions up to the highest beam energies currently available.

This experiment can be made fully operational by December 1994 since all the major detector systems exist and no large scale construction is needed. Our biggest effort will involve relocation and installation at BNL. The EOS TPC is a compact detector which does not require structural disassembly and it can be shipped as a single unit. It could be located at the $\mathrm{Al}, \mathrm{Bl}$, and $\mathrm{C5}$ beam line experimental areas. These beamlines are suitable for transporting heavy ions over the desired range of beam energies.

Most of the collaborators from Kent State, LBL, Purdue, U.C. Davis, and Texas A\&M were involved in the EOS experiments at LBL and have expertise in the TPC, MUSIC, and TOF detectors as well as in the DAQ, Beam Line Counters, Trigger, and Software. There is sufficient manpower to cover all detector elements.

The commitments of the LBL collaborators who are members of the STAR collaboration can only be finalized after the extent of the RHIC stretch-out is known. In the event of a RHIC stretch-out, the participation of all the above collaborators in this experiment at BNL would be beneficial to STAR.

The experiment will commence taking data with $\mathrm{Au}$ beam at $10.7 \mathrm{~A} \mathrm{GeV}$. At this energy we request 336 hours. In subsequent running periods we ask for.lower energy $A u$ beams: $8,6,4$, and $2 \mathrm{~A} \mathrm{GeV}$. This will allow us to study the energy dependence and find the point of highest compression. The time requested at these energies appear in the run schedule (see Section 6.4). 
This proposal is organized into six sections:

- Section 2 contains a detailed discussion of the physics.

- Section 3 outlines the proposed measurements.

- Section 4 is a brief introduction to the subject of critical phenomena in nuclear matter and its relevance to multifragmention studies.

- Section 5 presents a detailed account of the experimental apparatus.

- Section 6 briefly provides logistical information, available manpower, and the beam time schedule. 


\section{The Physics of Hot Dense Nuclear Matter}

\subsection{Nuclear Matter and the Equation of State}

An excellent review on compressed heated nuclear matter is given by K. H. Kampert [1] with an emphasis placed on the basic physical observables which guide our understanding of the reaction mechanisms, and on the discovery of strong collective phenomena in particle emission. We describe here the essential ideas and start by defining the meaning of the Equation of State (EOS).

Relativistic heavy ion collisions offer the unique opportunity to study the properties and response of nuclear matter under extreme conditions of temperature and density. If we had at our disposal bulk nuclaar matter we would libe to determine its EOS, that is, the thermodynamic response of tise substance to external forces. There exists a certain relation between the pressure, specific volume, and ten:perature which can be expressed in general as:

$$
f(P, v, T)=0 .
$$

This relation is known as the Equation of State of the substance. If any two of the properties are known, the third is fixed. This state frinction varies $f$ $m$ one substance to another and generally is quite complex in all but the simplest cases. For an ideal gas we obtain the well known Pv=RT law.

Now suppose we take a small volume of bulk nuclear matter, and ask for its the energy content. It will given by:

$$
E_{V}=\int_{\tau^{-}} \varrho(\mathbf{r}) \cdot W(\varrho(\mathbf{r}), T(\mathbf{r})) d V .
$$

It is this energy density functional $W(e, T)$ that is commonly called the Nuclear Matter Equation of State. It contains all the important thermodynamical features of bulk nuclear matter. In other scientific areas the EOS plays an important role in the static and dynamic behaviour of stars, especially supernova explosions and neutron star stability.

Experimental information about the nuclear EOS is largeiy restricted to densities and temperatures that exist close to its ground state. A speculative idea as to the nature of the function is often conveyed by a schematic phase diagram, such as the one shown in Fig. 1 . The vertical and horizontal axes represent the temperature and the normalized baryon density respectively. The nuclear matter EOS may well have a very rich and complicated structure. One recognizes different regions of the liquid-gas coexistence, resonance $(\Delta)$ matter, meson $(\boldsymbol{x})$ condensate, and the quark-gluon plasma. The intriguing possibilities of various phase transitions is continuing to receive considerable attention at AGS, CERN, and PHIC energies.

A commonly used form of the nuclear matter EOS stems originally from Skyrme. In its most simplified version this EOS is given by:

$$
W(\varrho, T=0)=E_{\text {kin }}+\alpha \varrho+\beta \varrho^{\gamma} .
$$

This equation tacitly assumes the EOS is separable into a linetic energy part and a potential energy part which is entirely datermined by the unknown parameters $\alpha, \beta$, and 
$(\mathrm{MeV})$

-

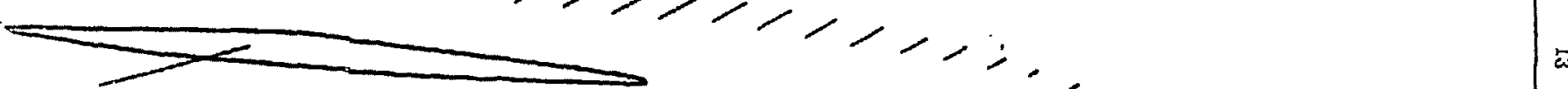

Traisition to massless plasma
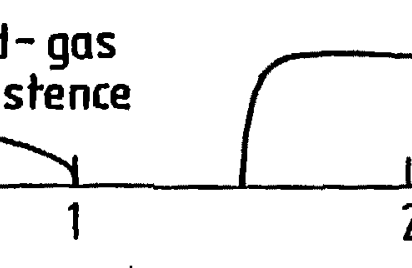

\section{Transition to}

$>$ delta-matter

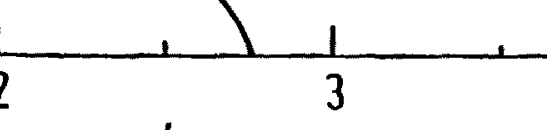

$\rho_{B} / \rho_{0}$

Figure 1: Phase diagram of nuclear matter. 


\begin{tabular}{llll}
\hline Reaction & $\begin{array}{l}\text { Threshold } \\
\mathrm{GeV}\end{array}$ & Reaction & $\begin{array}{l}\text { Threshold } \\
\mathrm{GeV}\end{array}$ \\
\hline$N N \rightarrow N N \pi$ & 0.29 & $N N \rightarrow N N K^{+} K^{-}$ & 2.5 \\
$\rightarrow N \Delta$ & 0.64 & $\rightarrow N \equiv K^{+} K^{+}$ & 3.7 \\
$\rightarrow N \Lambda K$ & 1.6 & $\rightarrow N N \tilde{N} N$ & 5.6 \\
$\rightarrow N \Sigma K^{\prime}$ & 1.8 & $\rightarrow N N \bar{\Lambda} A$ & 7.1 \\
\hline
\end{tabular}

Table 1: Laboratory thresholds for particle production in $\mathrm{N} \cdot \mathrm{N}$ collisions

$\gamma$. These parameters can be fixed in terms of the ground state properties of nuclear matter (i.e., the binding, energy and saturation density) and a new parameter called $K$, the nuclear compressibility. Large values of $K$ imply that it takes more energy to achieve compression, a situation corresponding to a hand or stiff EOS $(K \sim 380 \mathrm{MeV})$, while a small value $(K \sim 200 \mathrm{MeV})$ indicates a soft EOS. In Fig. 2, we show the nuclear compressional energy as a function of density for two different values of the compressibility factor.

\subsection{Resonance Matter}

Many inelastic scattering channels open up above $1 \mathrm{~A} \mathrm{GeV}$ and they should begin to play an increasingly important role in the collision dynamics. Some important thresholds in NN collisions are listed in Table 1.

The excitation of deltas is expected to soften the Equation of State. A soft EOS would imply less compression and therefore less collective matter flow. As the energy is increased, strangeness and antibaryon production thresholds are exceeded. Apart from being very useful probes these new channels compete for the available energy in the center of mass and, consequently, reduce nuclear compression.

At incident energies of 1-2 A GeV a gradual transition to resonance matter probably occurs, with $30 \%$ of the baryons located within the hot interaction zone being excited to $\Delta$ and $N^{*}$ states. This can be inferred from the the fact that the probability of producing a pion is abiut $30 \%$ at these energies. Furthermore, microscopic transport models $[2,3]$ indicate substa.tial stopping due to inertial confinement and that a baryou density, $\varrho_{b}$, of approximately 3 times normal nuclear matter density, $e_{a}$, is achieved in central collisions. This result implies a delta resonance density of 0.8-1.0 e.

For $\mathrm{Au}+\mathrm{Au}$ collisions at $5 \mathrm{~A} \mathrm{GeV}$, about $50 \%$ of all baryons could be excited resonz nces $\left(\Delta, N^{*}\right)$ reaching a maximum density of 2-3 times normal nuclear matter density for a duration of $10 \mathrm{fm} / \mathrm{c}$. This time is long compared to a typical NN collision time and thus a new state of nuclear matter - called "delta" or "resonance" matter - could conceivably be formed [4]. A large delta abundance would likely have a strong influence on the EOS which in turn would effect experimental observables such as the inclusive particle spectra and collective flow $[5,6]$.

Some relativistic mean field calculations [7] exhibit a second minimum in the nuclear 


\section{Compressional part of the EOS:}

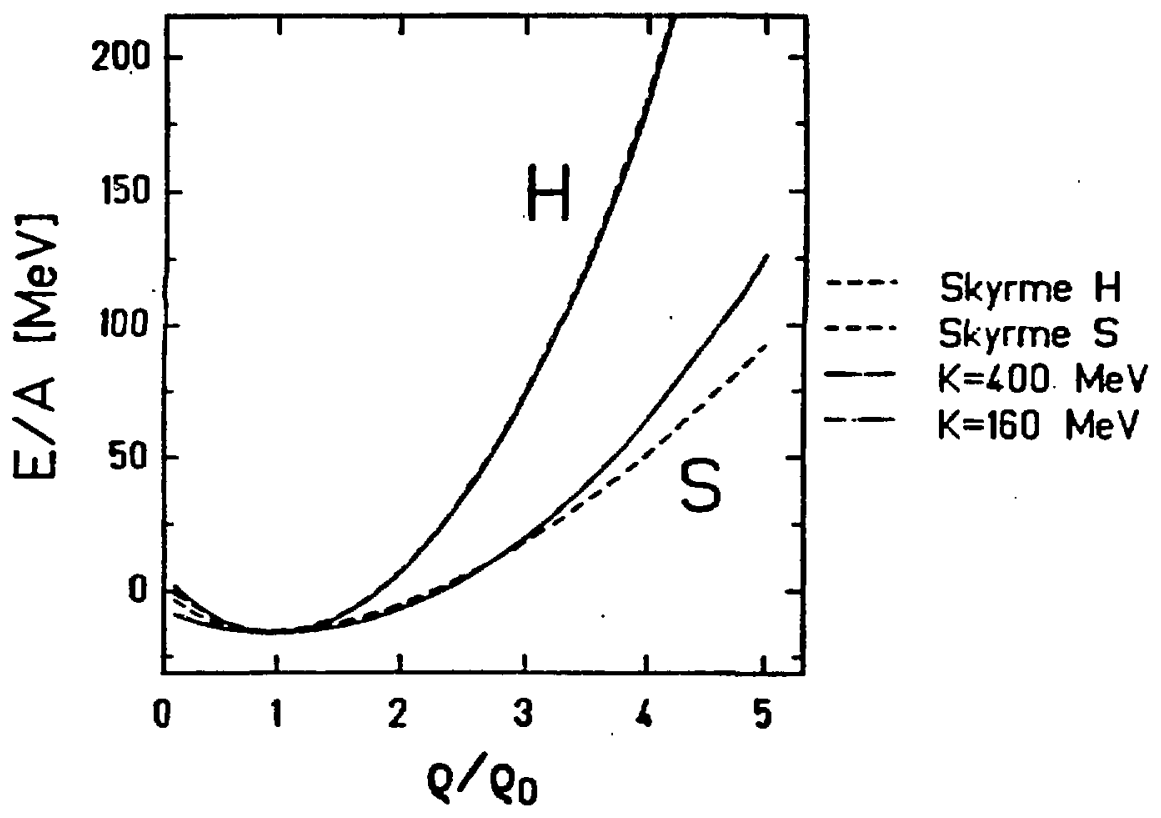

Figure 2: The nuclear compressional energy as a function of density for two different values of the compressibility factor $K$. 
EOS at high baryon density. This minimum, again, may be interpreted as the transition to resonance matter. Predictions of the EOS with different values of the delta coupling constants are shown in Fig. 3. The secondary minimum lies somewhere between baryon densities of 2-4 $\varrho_{0}$. Baryon densities ranging from 3-10 $\varrho_{0}$ are predicted to occur in central $\mathrm{Au}+\mathrm{Au}$ collisions in the beam energy region of 2-10 A GeV.

\subsection{Collective Phenomera and their Importance}

The isey mechanisms for producing hot dense matter were formulated first using hydrodynamical models. Scheid [8] argued that if nuclei stop each other one encounters a typical shock scenario where the participant matter cannot escape rapidly enough from the interaction zone, resulting in pile-up and nuclear compression. The shock front of highly compressed matter tries to escape and expand under a finite angle with respect to the beam direction. Thus, the principal signature of nuclear compression would be a preferential sidewards emission of particles, called "side-splash" or "directed flow".

This collective tendency of matter to be pushed aside during the expansion phase is illustrated in Fig. 4, which depicts a collision in the center-of-mass frame between two heavy nuclei. The reaction plane is defined as the plane containing the incident beam and the impact parameter, $b$. For central collisions $(b \sim 0)$ the flow is perpendicular to the beam axis. In semi-central collisions the flow angle lies between $0^{\circ}$ and $90^{\circ}$. Perpendicular to the reaction plane there is also a "squeeze-out" component in the c.m. frame. In that direction the matter can escape freely from the compression zone without being disturbed by the spectator material.

The ordered motion of an ensemble of nucleons as evidenced by collective flow can be regarded as a bulk property of suclear matter which carries information about its EOS. Nuclear matter flow is a direct measurement of nuclear compression and, with the aid of nuclear transport theories which model the dynamics of the collision, it can be related to the baryon density.

\subsection{Measuring Flow in Nuclear Matter}

Early experiments conducted at the Bevalac using $0.25-1.0 \mathrm{~A} \mathrm{GeV}$ heavy ion beams have proved the existence of collective fow in and out of the reaction plane. These results confirm that large nuclei are big enough for nuclear stopping and establish the formation of compressed nuclear matter.

Several methods are used to measure collective flow. We describe here the most widely used technique developed by Danielewicz and Odyniec [9] called the global transverse momentum analysis. In this method the reaction plane is constructed for each event from the transverse momentum components of all particles. Next, the transverse momentum vector of each particle is projected onto this plane, yielding the in-plane transverse momentum $p_{x}$, as shown in Fig. 5. For the sake of clarity, the ellipse represents the shape of the event in momentum space and it is rotated with respect to the beam to reflect the "directed flow". The average in-plane momentum per nucleon, $\left\langle p_{x} / A\right\rangle$, is then usually plotter. as a function of rapidity $(y)$ as shown in the bottom panel with actual data taken fror the EOS collaboration. 


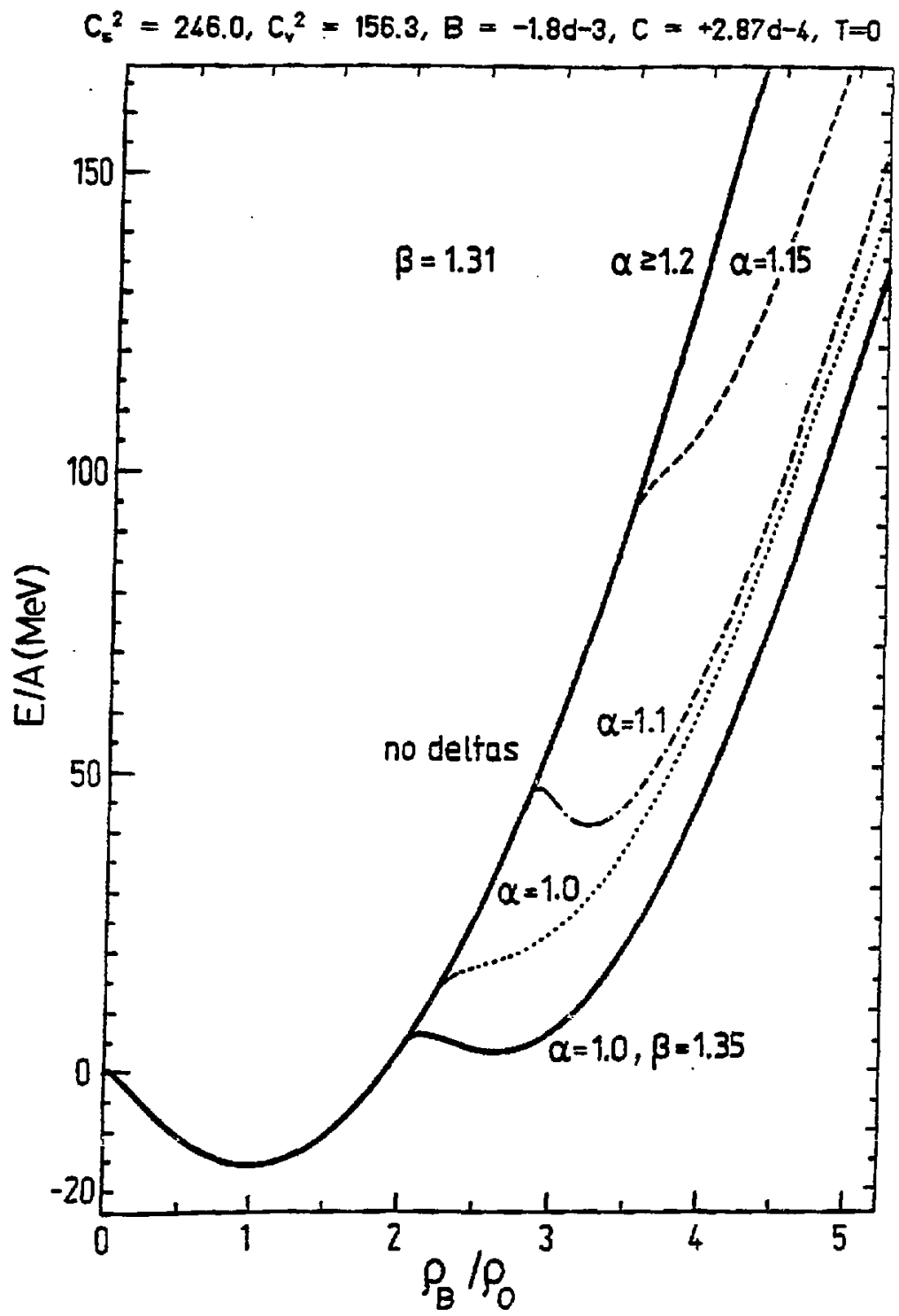

Figure 3: The exotic EOS including delta resonances at $T=0 \mathrm{MeV}$ for different values of the coupling constants $\alpha$ and $\beta$ as indicated. 

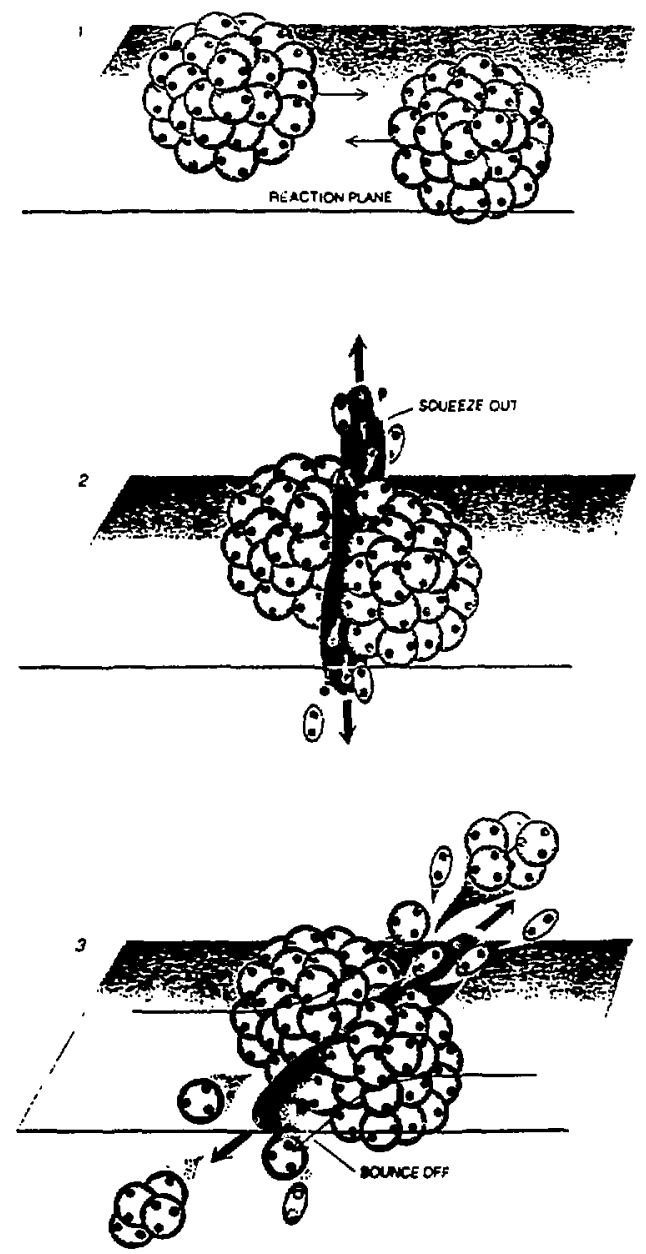

COUIDNC NUCD provide the key mectunism for investigating the nucker equa con of suit. Here wo zold auclei callide sliphtly af cenler (1) in the hol corroression zone formed by the sbock wave, maner composed of protons, piofs and other light paricles (perhaps quatks and gluons) is sapuezed out al tight angles to the $x$ accion plane (2). The remnants of the two nuetei then bounce of each other [3].

Figure 4: Schematic illustration of a nocleus-nocleus collision. 
The characteristic S-shape of the emerging curve is a clear sign of nuclear matter flow. The general features of such a distribution are: (a) particles close to the beam rapidity show a maximum flow and, (b) for the stopped, thermalized particles at mid-rapidity, the transverse mean momentum $\left(p_{x} / A\right)$ vanishes. The slope of the S-shape curve at midrapidity is often used to quantify flow. The distribution of $\left(p_{x} / A\right\rangle$ versus rapidity and $\left(p_{x} / A\right)$ versus beam energy are barometers of nuclear compression and therefore measure the pressure build-up between two colliding nuclei. The pressure in turn can be related to the density.

In the EOS experiments at the Bevalac, directed flow was measured separately for protons, deuterons, and other light mass particles as a function of beam energy up to the maximum possible linetic energy. The directed sidewards flow in At + Au collisions increases linearly as a function of incident energy up to the nlaximum beam energy of $1.15 \mathrm{~A} \mathrm{GeV}$. This represents the largest flow value observed in nuclear collisions to date. The inclusive flow excitation function is shown in Fig. 6 . The data are taken from the EOS collaboration and from the Plastic Ball group. We find directed flow grows stronget with increasing mass of the particle. The explanation is twofold. First, heavier fragments have more transverse momentum in the reaction plane than out of plane, in effect, causing preferential alignment. Second, the thermal velocity arising from the heat generated in the reaction zone is smaller for the heavier mass particles which show lower directed flow velocities.

The analysis of $\mathrm{Nj}+\mathrm{Cu}$ at $2.0 \mathrm{~A} \mathrm{GeV}$ data generally show a smaller value of flow as one would expect for a lighter system. The interesting result is that at $2.0 \mathrm{~A} \mathrm{GeV}$ the sidewards flow is still larger than at $1.0 \mathrm{~A} \mathrm{GeV}$. In other words, the dynamics which generate flow do not vanish at $2.0 \mathrm{~A} \mathrm{GeV}$. This underscores the importance of extending measurements continuously to the maximum AGS energy.

On the theoretical side, according to Janiche and Aichelin [10], an improved version of the Quantum Molecular Dynamics (QMD) transport model implies the sensitivity to the nuclear EOS for $\mathrm{Au}+\mathrm{Au}$ at $800 \mathrm{~A} \mathrm{MeV}$ is smaller than previously believed, and too small for flow in that system to be a useful barometer. Their conclusion is that the sensitivity should improve at higher beam energies since compressional energy is expected to rise and collective effects become more pronounced and easier to detect.

Very recently, a relativistic inter-nucieon cascade code called ARC [11] has successfully reproduced numerous data collected by several experiments for $\mathrm{Au}+\mathrm{Au}$ collisions at 11.6 A GeV/c. The ARC model does not contain an EOS and therefore does not make predictions about collective effects. However, the theoretical space-time evolution analysis indicate high nuclear densities are achieved in the presence of considerable stopping. It is argued that higher baryon and phase space densities can be anticipated at beam momenta lower than the present $11.7 \mathrm{GeV} / \mathrm{c}$.

In summary, recent and past $[12,13]$ experiments strongly suggest that we should expect to see greater flow above $2 \mathrm{~A} \mathrm{GeV}$. Theoretically, the 2-10 A GeV region is the most important for the hadron gas EOS if Aichelin's arguments are accepted. Likewise, the ARC model predicts maximum baryon density in this energy region. 


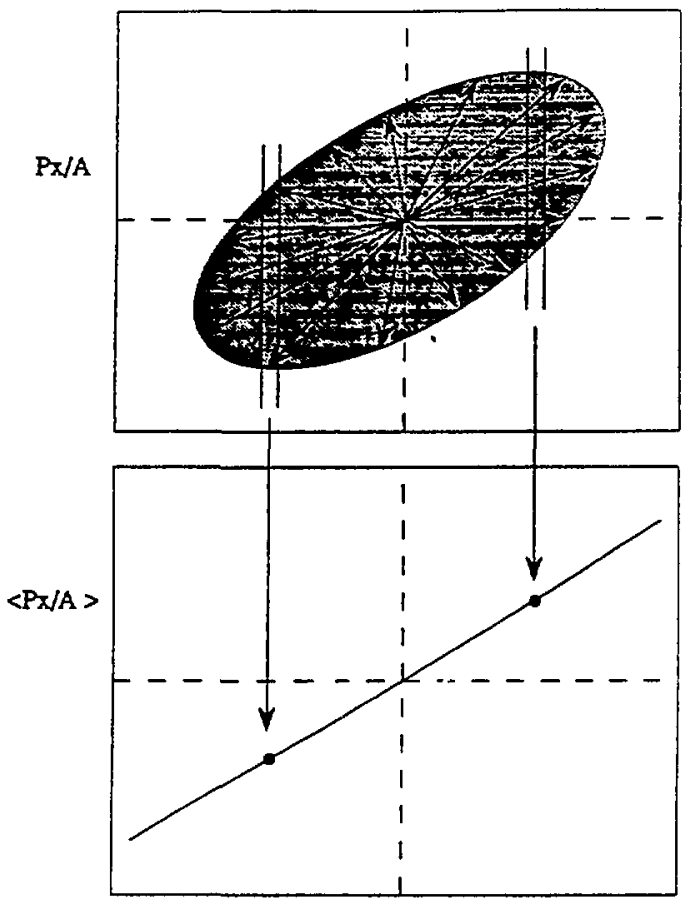

rapidity y

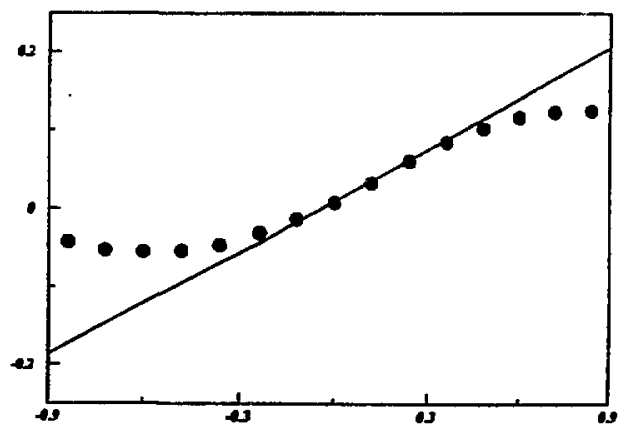

Figure 5: Transverse momentum analysis. 


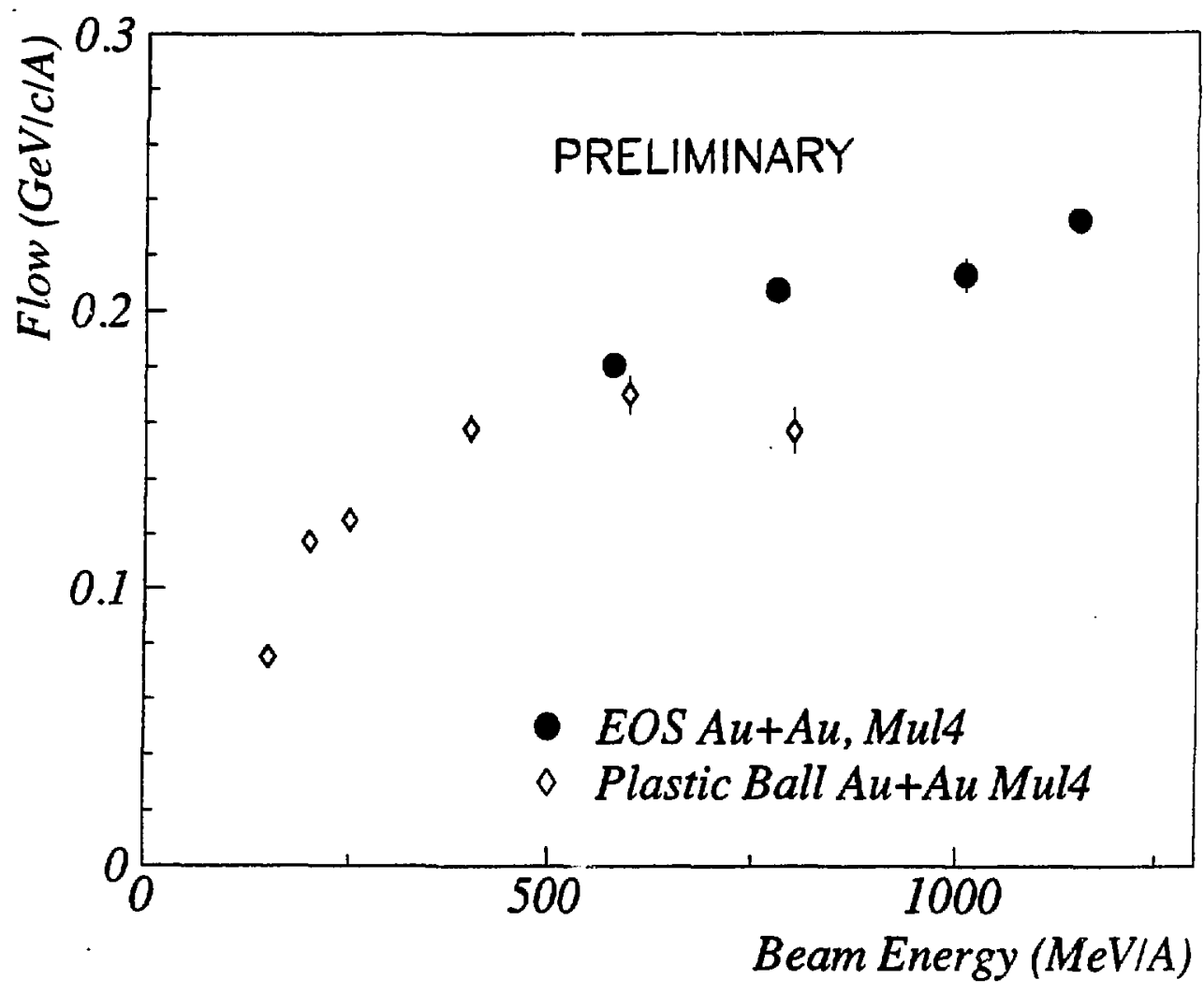

Figure 6: Flow parameters for the EOS data taken at the Bevalac for Au + Au. (These data are preliminary.) 


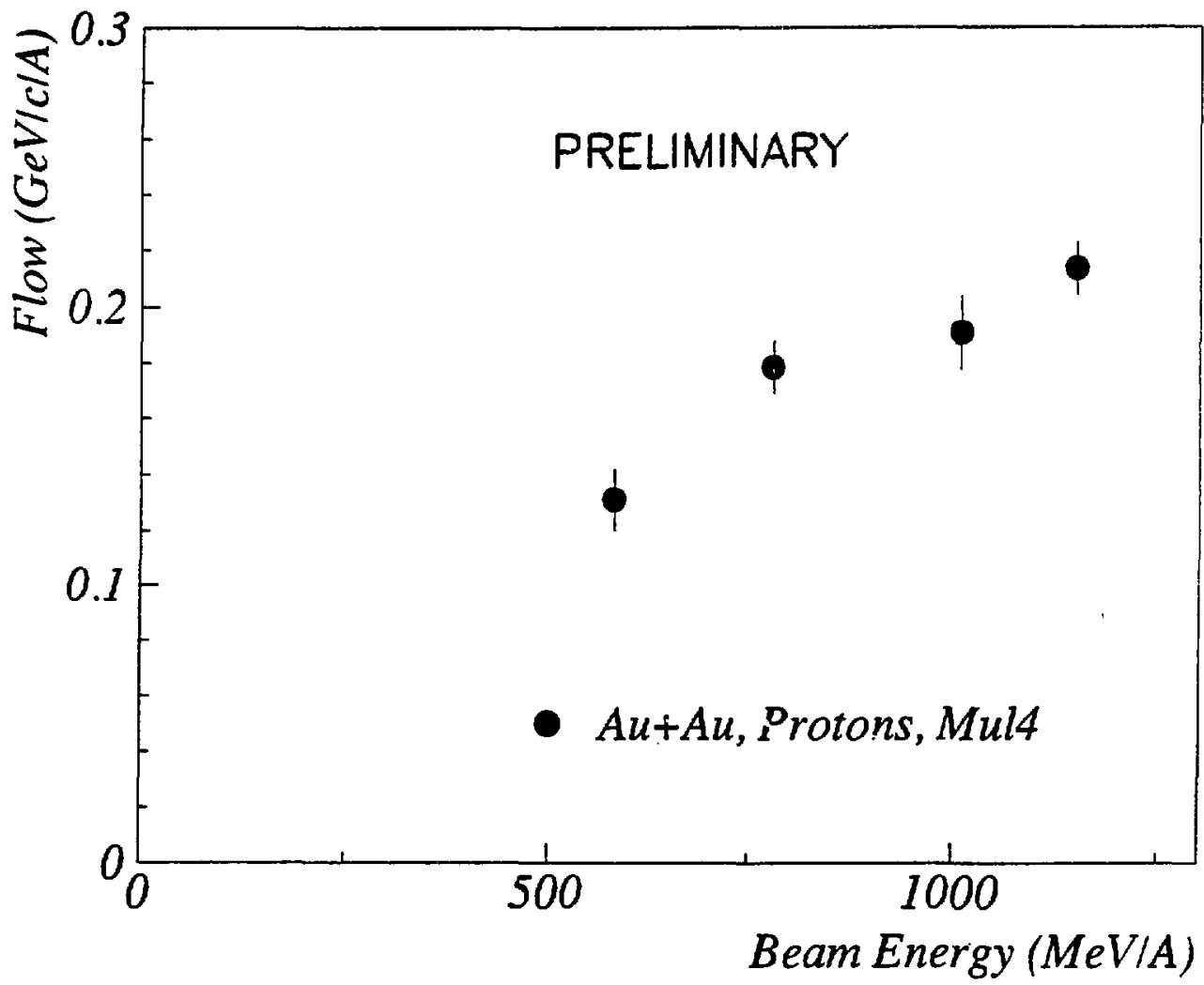

Figure 7: Flow excitation function for protons at the Bevalac. (These data are preliminary.) 


\subsection{Flow as a Signature of Quark-Gluon Plasma}

If highly compressed baryon matter is created in a collision, the system would undergo a rapid expansion and cause flow. However, if the energy density is ligh enough we may have a transition to the quark-gluon plasma (QGP). This matter, once created will also undergo a more rapid expansion due to the internal pressure arising from compressed gluon flux. The most obvious outcome of this flow is to impart an additional collective transverse momentum.

Nagamiya [14] has pointed out that the "effective temperature" observed in the E802 data satisfy:

$$
T_{0}(\mathrm{p})>T_{0}\left(\mathrm{~K}^{+}\right)>T_{0}(\pi)
$$

which is not inconsisient with the mass dependeace expected from hydrodynamical flow.

Very recent] y, the FNAL E735 pip experiment [15] measured values of $(p ;$ ) as a function of $d N_{c} / d \eta$ for antiprotons, laons, and pions. The data reveal the increase of $\left\langle p_{t}\right\rangle$ with $d N_{c} / d i$ iepends on the mass of the particle. This effect can be explained by the minijet model, however, it has also been associated with the presence of transverse flow of hadronic matter and might be indicative of the formation of the quark-gluon plasma.

Amelin et al. [16] studied collectivity in $160 \mathrm{~A} \mathrm{GeV} \mathrm{Pb}+\mathrm{Pb}$ collisions using the re]ativistic Fluid Dynamical Model (FDM). The FDM assumes local thermodynamic equilibrium and one of its input is an Equation of State. Two different physical scenarios were studied: (a) with a hadronic EOS and (b) with a QGP EOS. Their study suggests strong stopping and thermalization in heavy ion collisions at the AGS and CERN. The model predicts transverse and sideward fow in the reaction plane that is very sensitive to the EOS. For a purely hadronic EOS the maximur directed flow $p_{x}$ is around $0.4 \mathrm{GeV} / \mathrm{c}$ and smaller by a factor of two for the QGP EOS. The authors suggest this difference in directed flow provides a possibility to detect the formation of QGP and, particularly, its threshold energy. 


\section{Proposed Measurements}

It is extremely important to measure as many obsesrvables as possible on an event by event basis as a function of beam energy. Nuclear matter flow, particle production, rapidity dis. tributions, and inclusive spectra give information on the pressure, entropy, viscosity, and temperature; respectively. This simultaneous interrelation of many quantities will place extraordinary constraints on theories. The same argument holds for observing unusual events characteristic of a QGP.

\subsection{Collective Effects}

In order to predict the collective effects we have used the Relativistic Quantum Molecular Dynamics (RQMD) model [17, 18]. RQMD includes hard binary collision processes and a nuclear mean field potential.

Figure 8 shows a prediction for the reaction $\mathrm{Au}+\mathrm{Au}$ at $10.7 \mathrm{~A} \mathrm{GeV}$ bombarding energy. The in-plane transverse momentum distribution $p_{x}(y)$ for protons clearly exhibit the general s-shaped flow pattern seen at Bevalac energies. The absolute magnitude of the flow ( $p_{x} \approx 150 \mathrm{MeV} / \mathrm{c}$ at projectile rapidity) is however smaller than the highest value observed at lower energies for the same system.

Experiments have shown the flow of matter is stronger for heavier fragments and for this reason we compare, in Fig. 9, the flow of $A=2$ clusters with protons. The RQMD calculations show the same mass dependence. Because of the limited statistics we could not make any predictions for intermediate mass fragments $(Z>2)$, however.

In Fig. 10 we compare calculations done with and without (labeled as cascade) Iuclear potentials. The parameters have been adjusted so as to reproduce an equation of state with a ground state compressibility of $200 \mathrm{MeV}$ (soft EOS) or $380 \mathrm{MeV}$ (hard EOS). The cascade mode leads to a $p_{x}$ distribution, but the magnitude of the flow is almost doubled when the potentials are switched on. A clear difference between the soft and the hard EOS can also be observed. The absolute magnitude of the flow stays almost constant going dorin from 10.7 to $6 \mathrm{~A} \mathrm{GeV}$ bombarding energy.

As mentioned earlier, collective flow is sensitive to changes in the EOS. As an example, the consequence of an exotic EOS on the excitation function for directed flow is shorn in Fig. 11. The calculation has been performed using the VUU model for $\mathrm{Ca}+\mathrm{Ca}$ collisions at an impact parameter $b=2 \mathrm{fm}$. Results are shown for a conventional hard EOS and the exotic EOS (Fig. 3) with the parameters $\alpha=1.0, \beta=1.35$. In contrast to the hard EOS, the excitation function for the exotic EOS calculation shoris a rise followed by dip when a critical density (beam energy) is reached.

We plan to measure the nuclear matter flow for protons, deuterons, and other light mass parcicles. We will study the flow excitation function in order to determine the region of maximum baryon density and also look for abnormal behaviour in the EOS.

\subsection{Stopping}

Several proceedures have been used to determine the degree to which two colliding nuclej stop each other. The most obvious method is tc measure the rapidity distribution of the 


\section{$\mathrm{Au}(10.7 \mathrm{AGeV}, \mathrm{b}=3 \mathrm{fm})+\mathrm{Au}$ hard eos, no mdi}

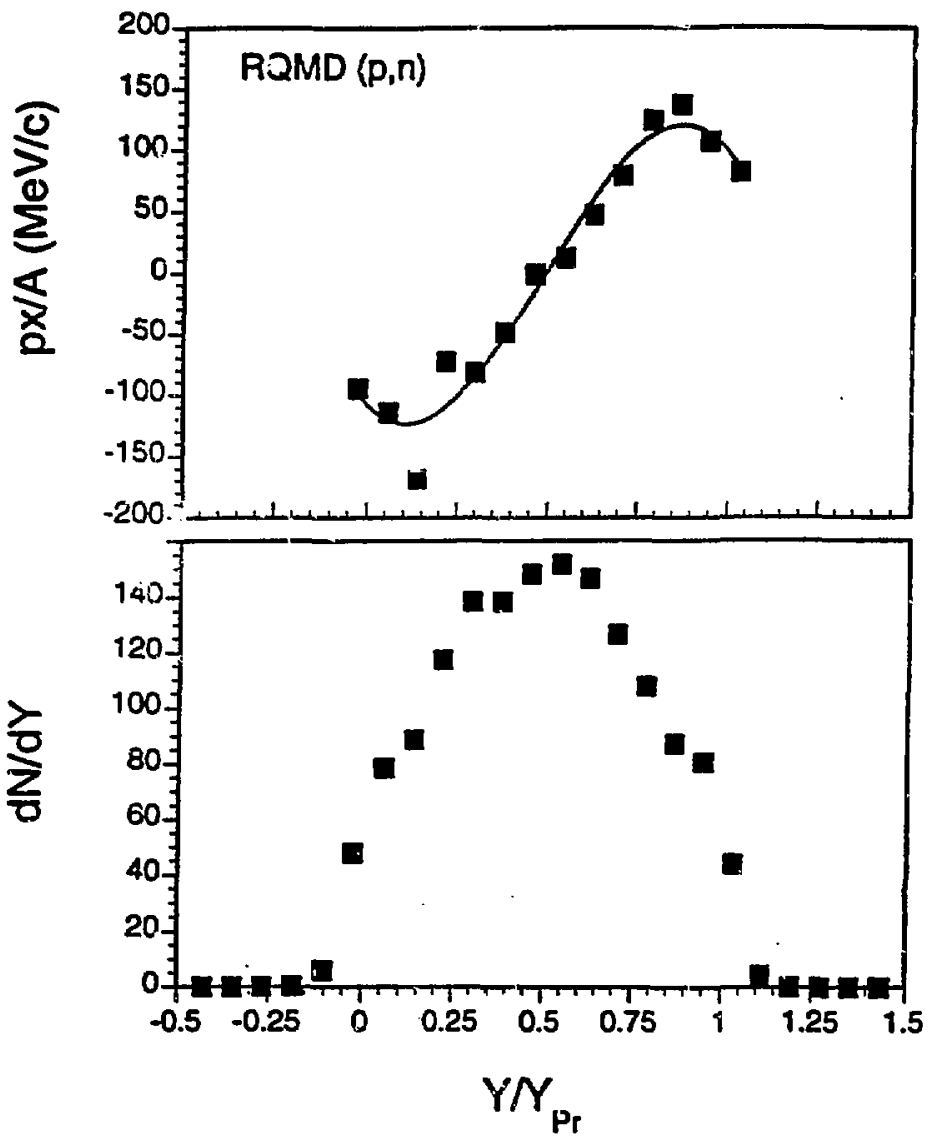

Figure 8: Transverse momenta for a RQMD simulation of $10.7 \mathrm{~A} \mathrm{GeV}$ (beam energy) Au $+\stackrel{4}{n .}$ 


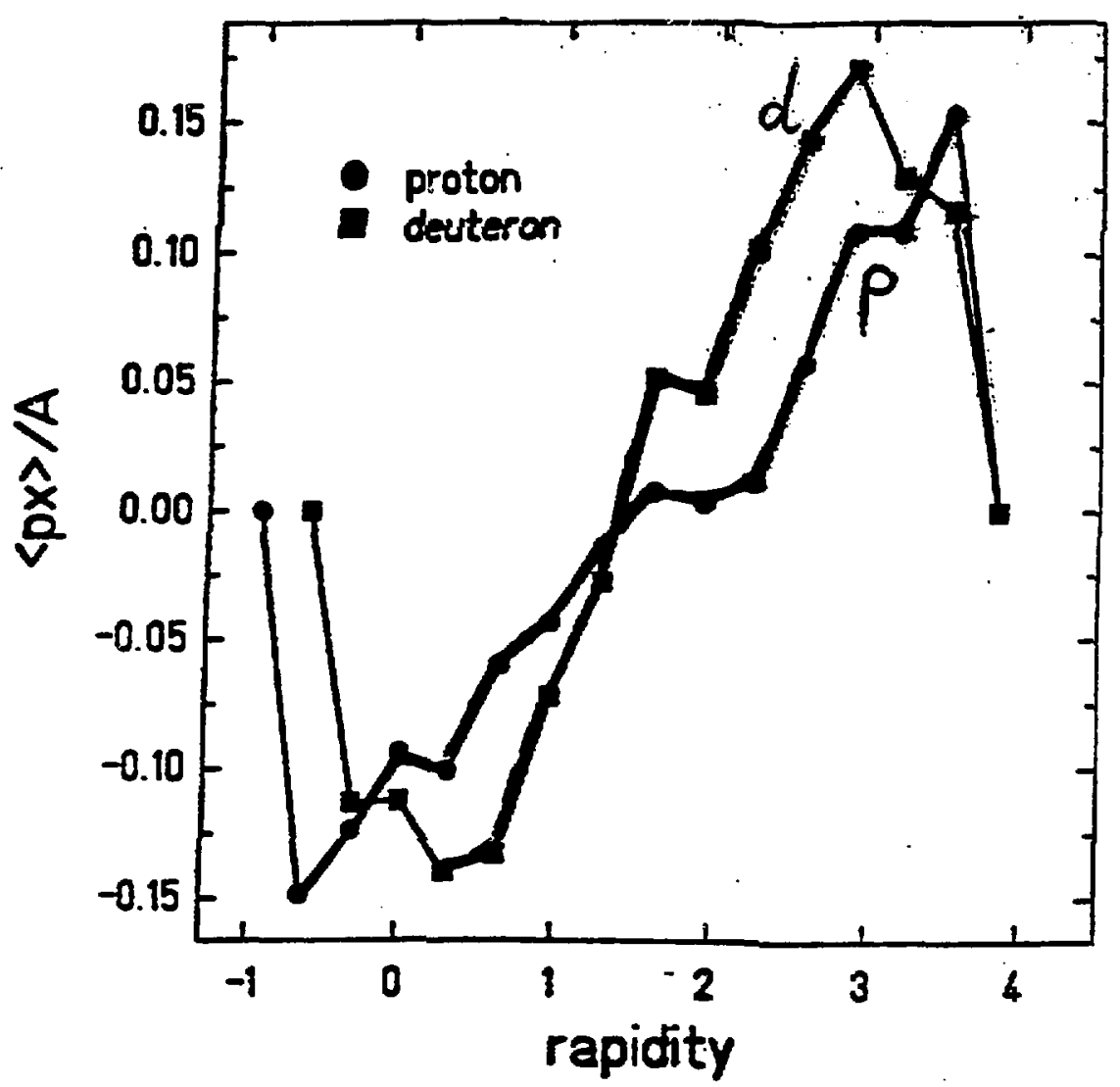

Figure 9: RQMD predictions for proton and deuteron flow for $10.7 \mathrm{~A} \mathrm{GeV} \mathrm{Au}+\mathrm{Au}$. 
$\mathrm{Au}(10.7 \mathrm{~A} \mathrm{GeV}, \mathrm{b}=3 \mathrm{fm})+\mathrm{Au}$

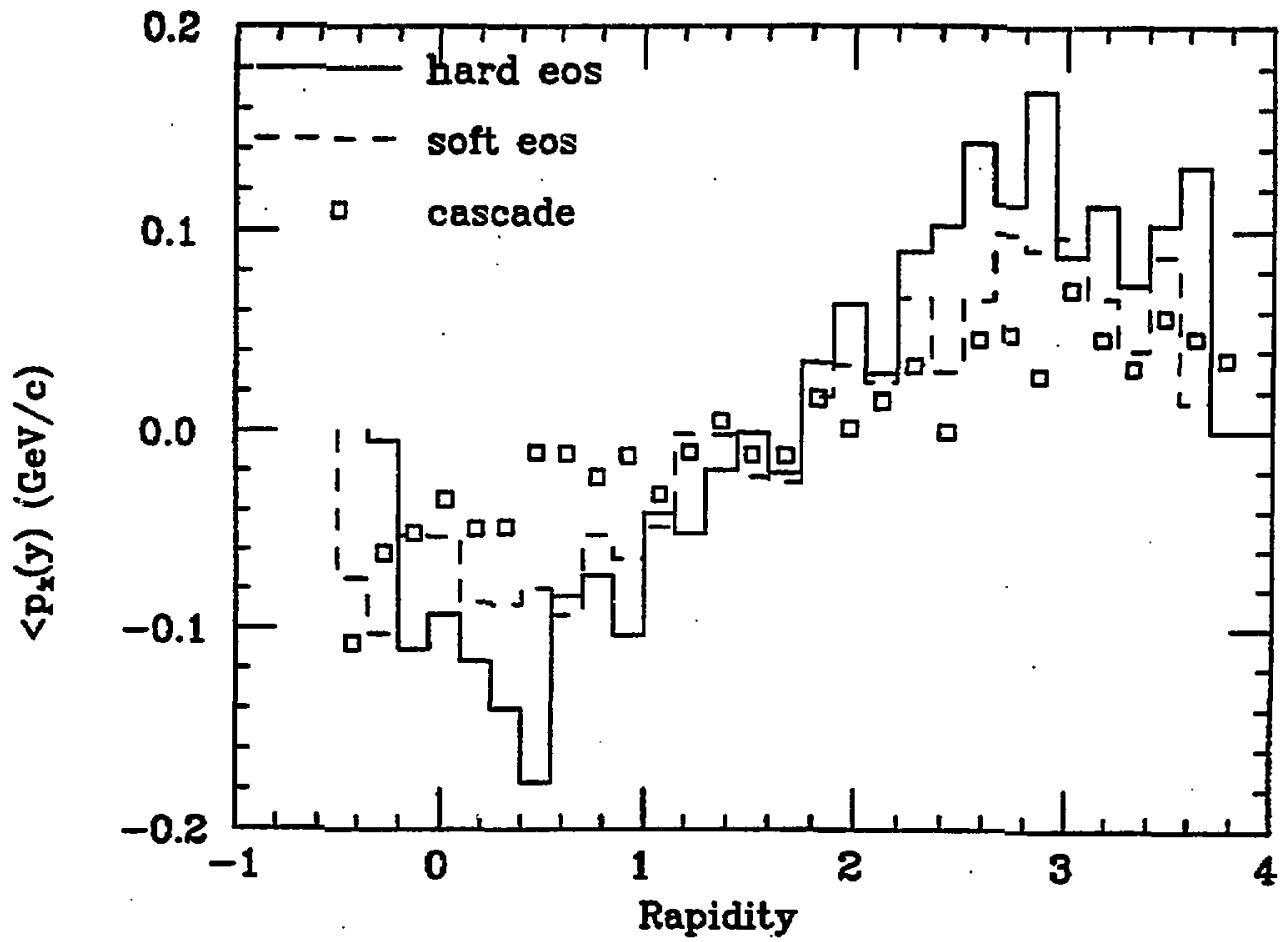

Figure 10: Flow for hard and soft equations of state at $10.7 \mathrm{~A} \mathrm{GeV}$ in the RQMD model. 


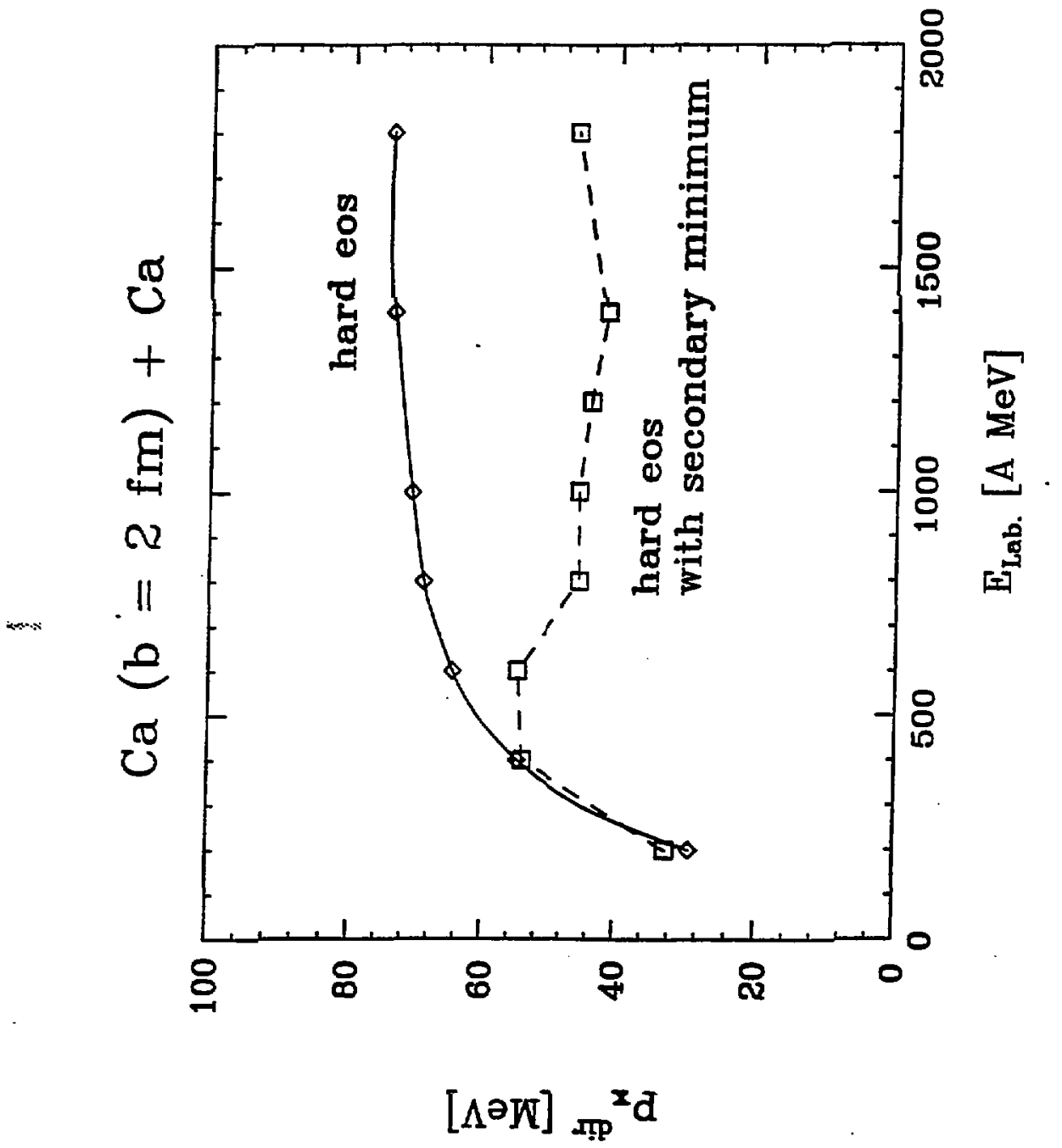

Figure 11: VUU calculation for the excitation function of the directed transverse flow $p_{x}$. The upper curve was obtained using the hard EOS, while for the lower curve the exotic EOS was used. 
protons emanating from the collision. Measurements by E802 and other collaborations indicate that a $14.5 \mathrm{~A} \mathrm{GeV} \mathrm{Si} \mathrm{projectile} \mathrm{is} \mathrm{fully} \mathrm{stopped} \mathrm{in} \mathrm{a} \mathrm{heavy} \mathrm{target} \mathrm{nucleus.}$

Another measure of stopping is the equipartition of energy among the transverse and longitudinal degrees of freedom. The stopping ratio $R$ is defined by

$$
R=\frac{\sum p_{\uparrow}^{2}}{2 \sum p_{\|}^{2}}
$$

$\mathbf{R}=1$ indicates complete stopping. This ratio can be determined without reference to any model, if the detector covers at least one half of the rapidity range. A detector with an acceptance that is not quite as large can still determine this ratio with a high confidence level.

Evidently, $R$ also measures the degree of thernalization since $R=1$ is a necessary, but not a sufficient condition for thermal equilibrium. We plan to do extensive measurements of the stopping ratio $R$ as a function of beam energy and impact parameter.

\subsection{Pion Production}

Pions are well suited to probe the properties of hadronic matter. The Diogene collaboration [19] analyzed the $\left(p_{x}\right)$ of pions to search for pionic collective flow. They found that $\left\langle p_{x} / A\right\rangle$ of the pions is anticorrelated with that of the protons. This observation has been connected to pion absorption in the spectator matter. A similar effect has been observed by $\mathrm{E} 802$ [20].

With RQMD model calculations Bass et al. $[21,18]$ have shown that the $\left(p_{x} / A\right)$ distribution is predominantly caused by pion scattering and is not a collective effect in the sense of the nucleon directed flow. The anticorrelation can only be observed in semicentral collisions since large pieces of spectator matter are needed to reflect the pions. Figure 12 sketches the two processes in the reaction plane.

The pion absorption picture needs to be studied at energies where a profusion of deltas are expected in the form of resonance matter. The measurement of a pion "flow-like" signal in conjunction with nuclear matter flow could be used to diagnose the properties of delta matter. The density of such matter is tied to the baryon density which we propose to vary systematically by reducing the beam energy in order to search for maximum nuclear compression. We will be able address the following issues:

1. How resonance matter affects the pion absorption.

2. How $\left\langle p_{x}(A)\right.$ for pions scale with energy.

3. Look for azimuthal correlations perpendicular to the event plane.

There is another interesting speculation. The rescattering of pions, including charge

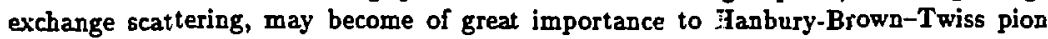
interferometry measurements just above Bevalac energies. The excited $N^{*}$ and $\Delta^{*}$ resonances can decay to stable nucleons with the emission of two pions, but not two identically charged pions (except $2 \pi^{\circ}$ ). Thus, the most easily measured $2 \pi^{-}$correlation may get an important contribution from $N^{*}$ decay accompanied by a charge-exchange pion scatter. There is perhaps already a hint of this in the Bevalac work of Bossy et al. [22], where 


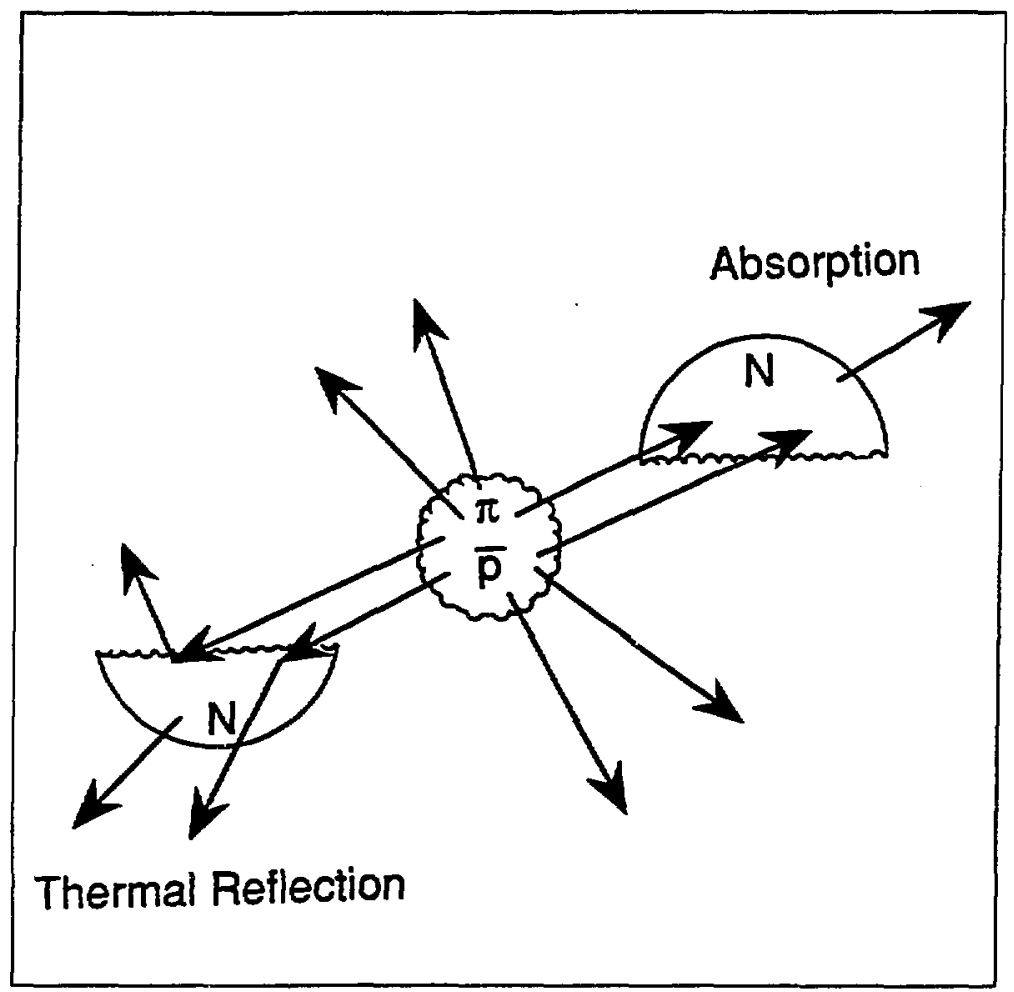

Figure 12: Schematic illustration of pion absorption and re-scattering by spectator matter. 
the pion source size parameter remains fairly constant for symmetric systems ranging in mass from 20 to 139 . One would expect the source size to increase as $A^{\frac{1}{3}}$ but perhaps the correlation is measuring some fixed distance - the mean free path for pion charge exchange scattering in spectator matter. This reasoning has far reaching consequences if the picture outlined above is correct. What does one measure when there is coexistence of significant delta and spectator matter?

\subsection{Strange Particle Production}

There is considerable interest in studying strange particle production with Au beams since the observed strangeness enhancement with the Si beam $[23,24,25]$ has been one of the most talked about results to come out of the AGS heavy ion program. While enhanced strange particle production has been suggested as a possible signature for quark-gluon plasma formation [26], purely hadronic models such as RQMD [27] and ARC [11] appear to be able to explain the increased yields as a manifestation of rescattering effects. How well the models do in predicting the preliminary strange particle data for $\mathrm{Au}+\mathrm{Au}$ collisions is an issue that is still being debated $[11,28]$.

Until recently less data were available on strange particle production at lower beam energies. Inclusive $K^{+}$spectra with $\mathrm{Ne}$ projectiles at $2.1 \mathrm{~A} \mathrm{GeV}(2.9 \mathrm{~A} \mathrm{GeV} / \mathrm{c})$ [29] have been measured. The production of $\Lambda$ 's in $1.8 \mathrm{~A} \mathrm{GeV}(2.6 \mathrm{~A} \mathrm{GeV} / \mathrm{c}) \mathrm{Ar}+\mathrm{KCl}$ collisions was studied at the Bevalac streamer chamber facility [30], albeit with limited statistics. The overall production rate ras cons:-itent with a simple nucleon-nucleon cascade picture but the momentum spectra showed features that may have indicated additional collective effects.

We propose to do a careful study of strangeness production $\left(K^{ \pm}, K_{a}^{\circ}, \Lambda, \Xi^{-}, \Omega^{-}\right)$from the top energy available with the $A u$ beam down to an energy that overlaps with our Bevalac data ( $\sim 2 \mathrm{~A} \mathrm{GeV}$ or $2.8 \mathrm{~A} \mathrm{GeV} / \mathrm{c}$ ). The physics issues that will be addressed include:

1. Strange particle production rates and $y$ and $p_{t}$ spectra. Other experiments will measure the ratio of strange to non-strange particles at $10.7 \mathrm{~A} \mathrm{GeV}$ but we will extend the measurements to lower beam energies. This will provide further constraints on the RQMD and ARC models.

2. $\frac{E^{-}}{\Lambda}$ and $\frac{\Omega-}{\Lambda}$ ratios. The production rate of doubly or triply strange objects may be greatly enhanced if a QGP is formed.

3. Azimuthal distributions of $\Lambda$ 's and K's with respect to the reaction plane in semi-central collisions. Azimuthal anisotropies have already been seen in the case of $\pi$ 's (see Section 3.3) and have been interpreted as being due to recattering by spectator matter. One would not expect to see the same effect for $\mathrm{K}^{+} \mathrm{G}$ since the scattering cross sections is smaller. There may be a flow signature in the $\Lambda$ 's, however, since the elastic $\Lambda \mathrm{N}$ cross section is of the same order of magnitude as the NN cross section.

4. $\mathrm{K}_{\mathrm{a}}$ interferometry. Neutral kaons offer many theoretical and experimental advantages over charged pions and kaons in HBT analyses. Resonance distortion is less severe for charged and neutral kaons [31] than it is for pjons. In the case 
of neutral kaons, Coulomb repulsion corrections don't have to be applied. Also, two-track resolution effects are much less important for $\mathrm{K}_{\text {: }}^{0}$ interferometry.

Simulations are currently under way to determine the ability of the EOS TPC to carry out these measurements. Relatively good identification of $K_{a}^{0}, s, \Lambda$ ' $6, \Xi^{-}$'s, and $\Omega^{-'} s$ can be made based on the topology of their decays alone. Identification of low momenta ( $p_{\text {iab }}<700 \mathrm{MeV} / \mathrm{c}$ ) $\mathrm{K}^{ \pm}$' 8 can be made on the basis of $d E / d x$ in the TPC. In addition, the identification of $K_{:}^{\circ} ' s, \Lambda^{\prime} \delta, \Xi^{-} ' \mathrm{~s}$, and $\Omega^{-}$'s may be considerably aided by partial $d E / d x$ identification (see Fig. 13). We are also studying the idea of using the TOF wall to augment our PID capabilities at mid-rapidity. The precise experimental configuration for strangeness measurements will be determined by GEANT simulations. Probably we would place the target as close to the active area of the TPC as possible in order to maximize our acceptance for particles produced backwards of mid-rapidity.

\subsection{Antiproton Production}

Antibaryons are an extremely useful tool for diagnesing the space time evolution of the hottest stage and for exploring collective procisses in nucleus-nucleus collisions. Antibaryon annibilation probes the local density and excitation energy as can be seen from the annihilation rate, $R_{a n n}=\left\langle\sigma_{a n n} \cdot v_{r e l} \cdot f_{B}\right\rangle$, where $f_{B}$ denotes the baryon phase space density. Various mechazisms of antibaryon enrichment have been proposed e.g., hadronic multi-step processes, string-string interactions, the formation of QGP, and strong mean field effects. On the other hand, suppression can provide information about the presence of large net baryon density and the reaction times [32, 33, 34].

In 1990, the E802 experiment [35] presented preliminary antiproton measurements of $d N_{\mathrm{p}} / d y$ for $14.6 \mathrm{~A} \mathrm{GeV} / \mathrm{c} \mathrm{Si}+\mathrm{Al}$ central collisions. Since that time there has been tremendous interest in the data and, recently, much debate. The reason is that two different physics mechanisms have been proposed which appear to explain the data equally well.

In the ARC model [11], which describes a large variety of AGS data, the initial production of antiprotons arises from the first, high energy collisions between the target and projectile nucleons. However, annihilation of antiprotons by the surrounding medium is strongly suppressed by a three-body screening mechanism [36].

The RQMD model [17], on the other hand, predicts the initjal formation of antiprotons is considerably enhanced by collective multi-step processes. In this approach, strings are created with rnasses not accessible in pp reactions and collisions between two highly excited resonance states are allowed to occur in the collision. The soup of resonances and strings may be further excited in subsequent collisions to a mass larger than $3 m_{\mathrm{N}}$ which allows for $N \bar{N}$ creation. However, it turns out that in the RQMD model $\sim 65 \%$ of the produced antiprotons are annihilated by the surrounding baryon rich matter. This cancellation of two large effects leads to agreement with the E802 data.

If antiprotons are absorbed by the surrounding spectator matter then, in complete analogy with the pions discussed in section 3.3, one should expect a "flow-like" signature for the antiprotons, anticorrelated with genuine nuclear matter flow. In Fig. 14, we present the RQMD calculation [18] for $\left(p_{x} / A\right)$ vs. rapidity $\left(y / y_{p}\right)$ for $\mathrm{Au}+\mathrm{Au}$ at $10.7 \mathrm{~A} \mathrm{GeV}$ which show such an anticorrelation signal. 
10.7A GeV Au + Au

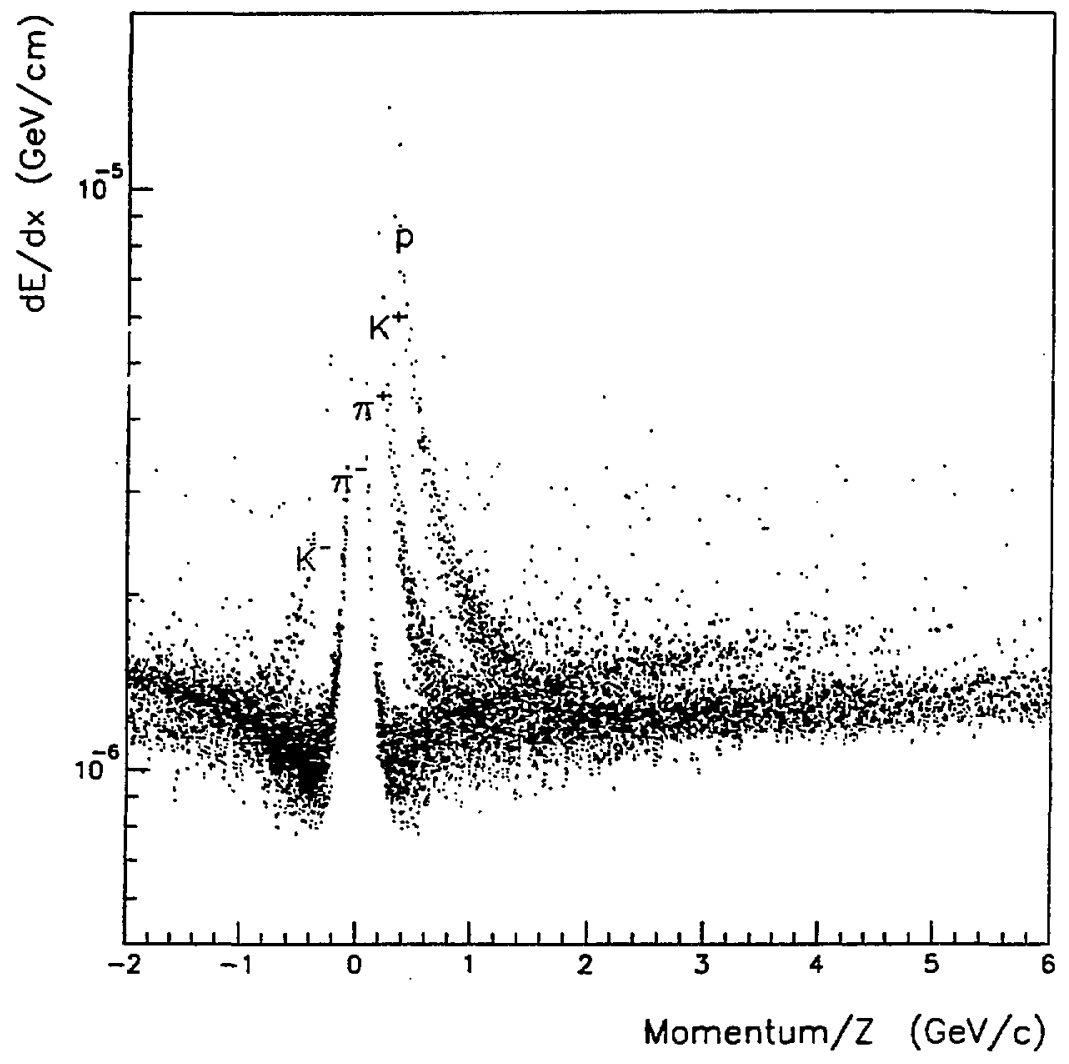

Figure 13: Reconstructed $d E / d x$ vs $p / Z$ of strange primaries and their $p$ and $\pi$ decay products from $20010.7 \mathrm{~A} \mathrm{GeV} \mathrm{Au}+\mathrm{Au}$ ARC events. A cut requiring at least 20 hits in the TPC has been applied. 
For the $10.7 \mathrm{~A} \mathrm{GeV}, \mathrm{Au}+\mathrm{Au}$ reaction we plan to acquire $5 \times 10^{5}$ semi-central triggers in 242 hours of beam time. Using the E802 results we anticipate a $\bar{p}$ yield in excess of $5 \times 10^{4}$ covering the same acceptance $\left(1.0 \leq y \leq 1.6,0.3 \leq p_{t} \leq 0.8\right)$. This should be sufficient to measure aximuthal asymmetries of $\overline{\bar{p}}$ 's with respect to the reaction plane. Such measurements may allow one to get a direct handle on the antibaryon production mechanism and to disentangle the relative importance of the competing effects of creation and annibilation.

In our experimental setup the $\overline{\mathrm{p}}$ measurements will be made using the EOS TPC detector alone. The TPC has a broad tracling acceptance covering the region $0.5 \leq$ $y \leq 2.5,0.3 \leq p_{t} \leq 2.0$. However, unambiguous proton particle identification limit our measurements to backward rapidity $(y \sim 1.0)$ ai the higher beam energies. This will also be our minimum acceptance for $\bar{p}$. We have not yet studied the optimum arrangement of the TPC in our simulations for measuring just the negatively charged particles. It may be possible to extend the particle identification capability at mid-rapidity using the TOF wall.

\subsection{Intensity Interferometry}

Intensity interferometry, also called the Hanbury-Brown-Triss (HBT) effect has become a well established technique for studying space-time evolution of nuclear collisions [37]. E802 finds the radius of the emitting source satisfy $R_{\pi *}>R_{p p}$. This fact taken together with the observation that the apparent source temperature for pions is lower than for protons, suggests the proton distribution is characteristic of an earlier, hotter stage of the collision whereas the pions reflect the conditions prevalent at a later, cooler stage. The $\mathrm{K}^{+}$meson has a long mean free path in nuclear matter which suggests it would be a useful probe of a still earlier, hotter, and more dense stage of the collision. In general, particles emitted with longer mean free paths will sample the collision phase of higher energy density and thus tend to carry higher kinetic energies. Thus, each type of produced particle records a different time of the nuclear collision and serves both as a nuclear clock and a thermometer.

Most HBT experiments have measured correlations of $\pi^{-}$pairs, although there have been some measurements with $\pi^{+}$and proton pairs. Recent AGS experiments have dedicated measurements on twc-kaon, two-pion and two-proton correlations. These comparative studies are necessary to obtain complete information on dynamics. The E802/859/866 Collaboration [38] has recently demonstrated that the RMS radii extracted from twoproton correlation functions measured for central $\mathrm{Si}+\mathrm{Al}, \mathrm{Ag}, \mathrm{Au}$ collisions at beam moment um of 14.6 A GeV/c scale with the RMS radii for the participant collision volume.

The extraction of information about the space-time structure of the emitting source through HBT correlations is considerably complicated by final state interactions and resonance formation. Kaons offer significant advantages over pions in these regards $[31,39]$; in particular resonance production increases the apparent size and lifetime of the source, and this distortion is less severe for charged and neutral kaons. Moreover, independent HBT measurements for $\mathrm{K}^{+}$and $\mathrm{K}_{\mathrm{a}}^{\circ} \mathrm{can}$, in principle, remove the resonance distortion. Neutral kaons offer the additional important advantage that no corrections need to be 


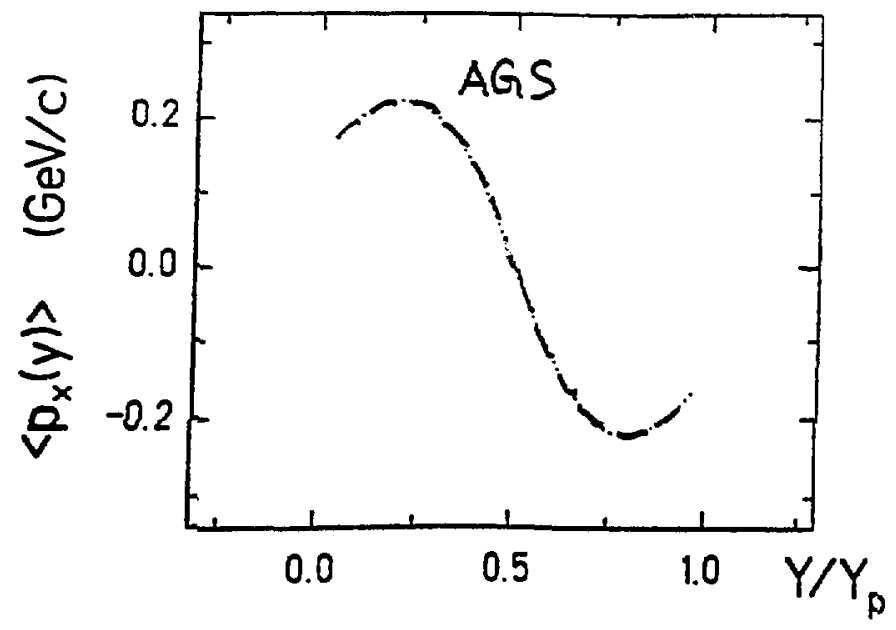

Al14.6 $\mathrm{AGeV} / \mathrm{c}$ )+A (central)

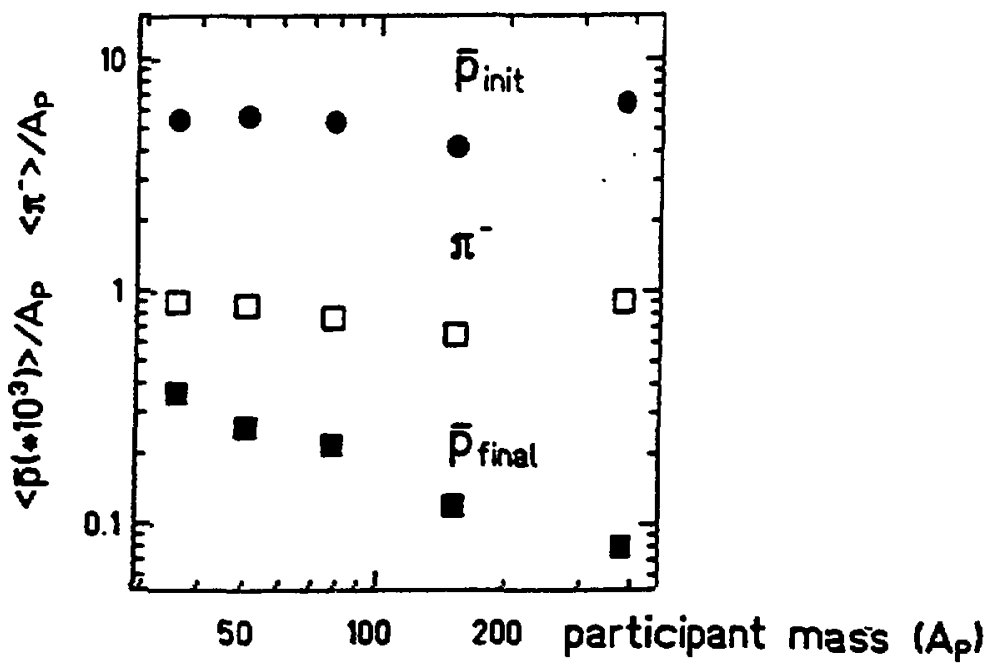

Figure 14: Top: Anti-proton "Đow-like" signature (transverse momentum vs. rapidity) as predicted by RQMD for minimum bias Au+Au at $10.7 \mathrm{~A} \mathrm{GeV}$. Bottom: RQMD prediction for anti-proton production yields as a function of participant mass 
made for final state Coulomb interactions. Also, some interesting and potentially important consequences arise from the fact that the $\mathrm{K}_{a}^{\circ}$ is not a strangeness eigenstate. The

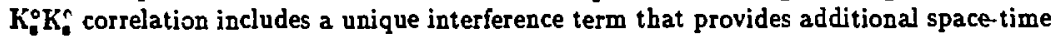
information [39] as well as insight into possible strangeness distillation effects $[39,40]$ in cases where the baryon density of the source is appreciable.

We plan to do systematic measurements on $\pi^{ \pm}, \mathrm{p}, \mathrm{K}^{+}$, and $\mathrm{K}_{\mathrm{p}}^{\circ}$ pairs and correlate the information with other global observables. The $\mathrm{K}_{a}^{\circ}$ particles can be identified on the basis of their decay topologies. Their production rates in central $A u+A u$ collisions should be large enough that sufficient statistics can be accumulated in a short running period - even allowing for detector inefficiencies (ARC predicts $\sim 20 \mathrm{Ka}$ 's per event at $10.7 \mathrm{~A}$ $\mathrm{GeV}$ and $\sim 10$ per event at $5 \mathrm{~A} \mathrm{GeV}$ ). For $\pi^{ \pm}, \mathrm{p}$, and $\mathrm{K}^{+}$pairs we will have to rely on particle identification from $d E / d x$ measurements in the TPC and possibly from the TOF wall. This will restrict our acceptance to pairs with low momenta in the lab but otherwise should cause no serious problems.

\subsection{Composite Fragment Production}

Light composite particles are important because they contain information about the following:

1. Freeze-out temperature or final state interactions.

2. Entropy: if entropy stays constant during the expansion phase of the collision, then it conveys information about the initial, highly excited, and compressed stage of the reaction. Moreover, possible phase transitions would be perceptible by their sudden liberation of new degrees of freedom.

3. Flow: collective flow influences are stronger for heavier mass fragments. Light mass particles convey information on compression and hence the nuclear density. Figure 9 shuws the predictions for proton and deuteron flow at the AGS for the $\mathrm{Au}+\mathrm{Au}(10.7 \mathrm{~A} \mathrm{GeV})$ reaction. The slope at mid-rapidity is greater by almost a factor of two.

4. Coalescence: Statistical coalescence predicts the composite fragment phase space distribution based on the primordial distribution of the nucleons. This scheme is independent of the collision dynamics and the formation mechanism. Final state coalescence is obtained by clustering nucleons at freeze-out. The lat ter reproduces the transverse momentum spectra at Bevalac energies. Light fragment yields can be used to test the coalescence models, which are in turn used to predict exotic composites.

The E802 experiment [41] reported a hint that many events involve multiple fragments (multifragmentation). In Fig. 15 we show the fragment yield distribution obtained for the 10.7 A GeV Au + Au reaction using the RQMD model [42]. The fragment yield follows a power law distribution with exponent $\tau=3.75$. The EOS TPC has very good particle identification for light mass fragments (up to $Z \sim 5-6$ ) and we will be able to easily measure the heavier mass nuclei $(Z \geq 7)$ with the MUSIC and TOF detectors. 

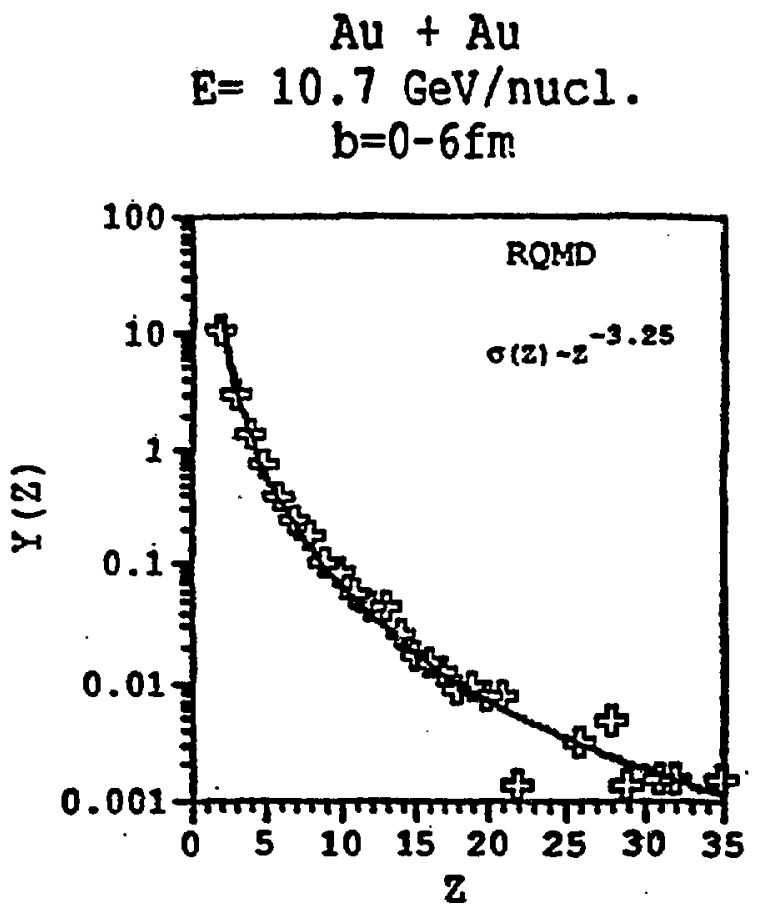

Figure 15: Fragment yield distribution for $10.7 \mathrm{~A} \mathrm{GeV} \mathrm{Au}+\mathrm{Au}$. 


\section{Physics of Dilute Matter}

In the previous sections we concentrated on the physics of dense nuclear matter. Also of interest is the behaviour of dilute nuclear matter in the vicinity of a phase transition. We expect that at a temperature of about 6 to $10 \mathrm{MeV}$ a liquid-gas phase transition tales place in infinite nuclear matter via spinodal decomposition or cavitation. When this happens, the EOS is described by a set of numbers called the critical indices which characterize the sharp change in the properties of a substance.

Nuclear multifragmentation is believed to have many similarities with a phase transition and consequently it is a fast-developing field of statistical mechanics. The essential idea is that the fragment size distribution and related quantities are scale invariant. This realization paves the way for model independent analyses in terms of scaling properties.

\subsection{A General Overview of Multifragmentation}

1. Substantial evidence now exists that nuclear multifragmentation is a two step process $[43,44,45]$.

2. Recent experiments have focused on extracting the values $o_{1}$ the critical exponents describing the details of critical phenomena ( $r$, etc.).

3. Little effort has been made to understand the dynamics of the energy deposition mechanism and the transition from evaporation to multifragmentation.

4. There are theoretical models describing the dynamics $[46,47]$ but there are no exclusive experimental data that cover the transition region from evaporation to multifragmentation. This is the most interesting region to study, since the dynamics change significantly.

5. The jdeal way to understand the dynainics would be to study proton-nucleus reactions, using reverse linematics in the energy regime of 1-5 A GeV. We know from inclusive $p$-nucleus studies in this energy regime that there is a large multifragmentation component $[48,49,50,51]$.

A TPC experiment with complete charge reconstruction would permit a determination of the mean energy deposited into the heavy nucleus as a function of beam energy and event multiplicity. This is a quantity that can be compared to theory, and it is the quantity that is most relevant in a phase transition picture. We will obtain a more detailed understanding of the two-step reaction process, that is, an initial stage followed by the multibody brealiup of the remaining nucleus. Inclusive studies indicate that the system that ultimately fragments is reduced in nucleon number from its initial value. This reduction is due to the knock-out of nucleons in the initial stages of the reaction. Calculations of this process compare favorably to the limited inclusive data that exists $[46,47,52]$. It. is clearly important to understand the role this initial stage plays in driving multifragmentation. An exclusive experiment at the AGS would have the capability of separating the initial stage nucleons from the fragmontation nucleons, thus permitting a determination from the apportioning of energy between these two stages. Such an experiment could therefore address detailed dynamical questions and would significantly constrain theoretical descriptions of the energy deposit ion process in nucleus-nu leus collisions. 
Because nuclear multifragmentation is a test bed for the study of the thermodynamics of small systems, we include a short synopsis of the evidence for . tical behavio: in this process. Receni experiments at the Bevalac have focused on extracting the critical exponents, $\tau$ and $\sigma$. We now know that in the first step of a proton-nucleus collision, nucieons are scattered by the projectile. As the scattered and produced particles leave the nucleus, they deposit energy on the order of the nuclear binding energy. Following this, the excited nuclear remnant expands and finally fragments. The focus of this proposal is on the energy deposition mechanism which drives the critical phenomeza.

\subsection{Nuclear Fragmentation as a Critical Phenomenon}

A schematic pisture of characteristic mass spectra produced whea a nucleus $A_{T}$ is bom. barded by protons is shown in Fig. 16. For energy depusition low compared to the total binding energy $E_{2}$, the mass yield, $\mathrm{Y}\left(A_{F}\right)$, (a) reflects the evaporation of nucleons and ight particles. For very high energy deposition compared to $E_{b}$, the mass spectrum (c) indicates total vaporization. The intermediate case (b), where the energy deposition is on the order of $E_{b}$, indicates the presence of fragments $1<A_{F}<49$ whose yield at the critical point would have power law behavior $1 / A_{F}^{\top}$, where $\tau \sim 2.2$. Experimentally $\mathrm{Y}\left(A_{F}\right) \propto A_{F}^{T}$ has been seen in a variety of experiments. The power law dependence is consistent with critical behavior. In simplest terms, the !ighly excited nuclear system is torn between being a liquid (clusters ir fragments) or being a vapor (nucleon.s). Cleaily such behavior depends on the temferature and density of the fragmenting system. Near the critical point, the system is self-similar and the residual effects of a phase transition in infinite matter may persist to the smallest length scales. Such a systam would undergo a Coulomb disassembly, leading to multifragmentation. The best known macroscopic example is critical opalescence.

In an exclusive multifragmentation experiment, the fragment sizes must be determined on an event-by-event basis. In practice, determining the fragment charge $Z$ is sufficient. Fragment distributions are characterized by moments of the distributions $[53,54,55,56]$

$$
M_{k}=\frac{\sum_{n} Z^{k} \cdot N(Z, n)}{Z_{0}}
$$

where $N(Z, n)$ is the number of fragments with $Z$ in an event of reduced multiplicity $n=m / Z_{o}, m$ is the measured charge multiplicity, and $Z_{o}$ is the charge of the emnant. The determination of $M_{k}$, the measured moments, is the goal of this experimont. To illustrate these model independent methods we use the results of scaling theory [57].

$$
M_{k} \sim\left(n-\pi_{c}\right) \frac{-(1+h-v)}{r}
$$

when $|n-n c| \rightarrow 0$. Plotting $\ln \left(M_{3}\right)$ vs $\ln \left(M_{2}\right)$ and computing the slope we find

$$
\frac{\Delta \ln \left(M_{3}\right)}{\Delta \ln \left(M_{2}\right)}=\frac{4-T}{3-T}
$$

The results from a 5000 event percolation simulation for a 108 nucleon system is shown in Fig. 17. Using a multiplicity cut to extract events in the critical region, we obtain the inset and fitting the slope graphically we obtain $r=2.1 \pm 0.1$. 


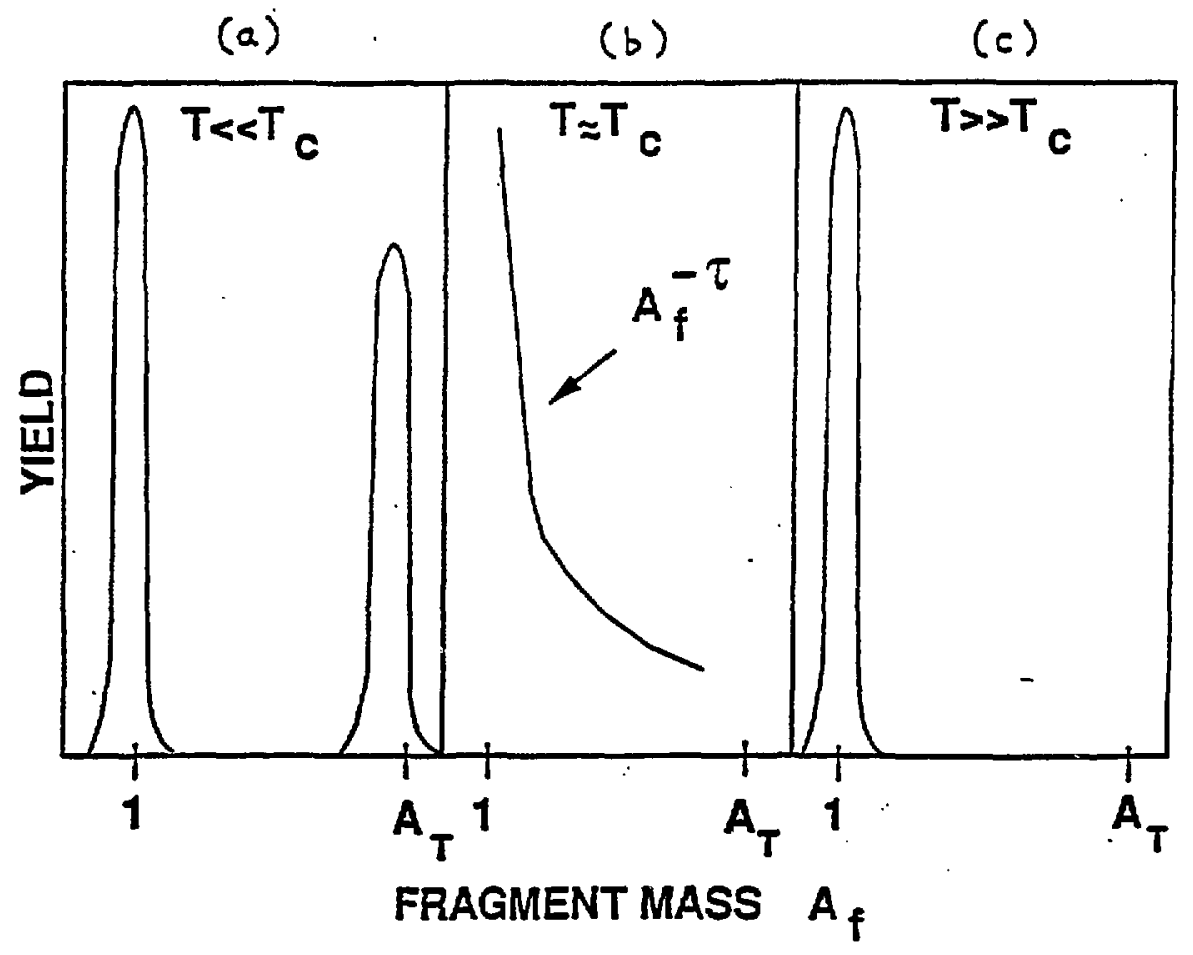

Figure 16: Schematic of mass spectra for $T<T_{e}, T \sim T_{e}$, and $T>T_{c}$. 


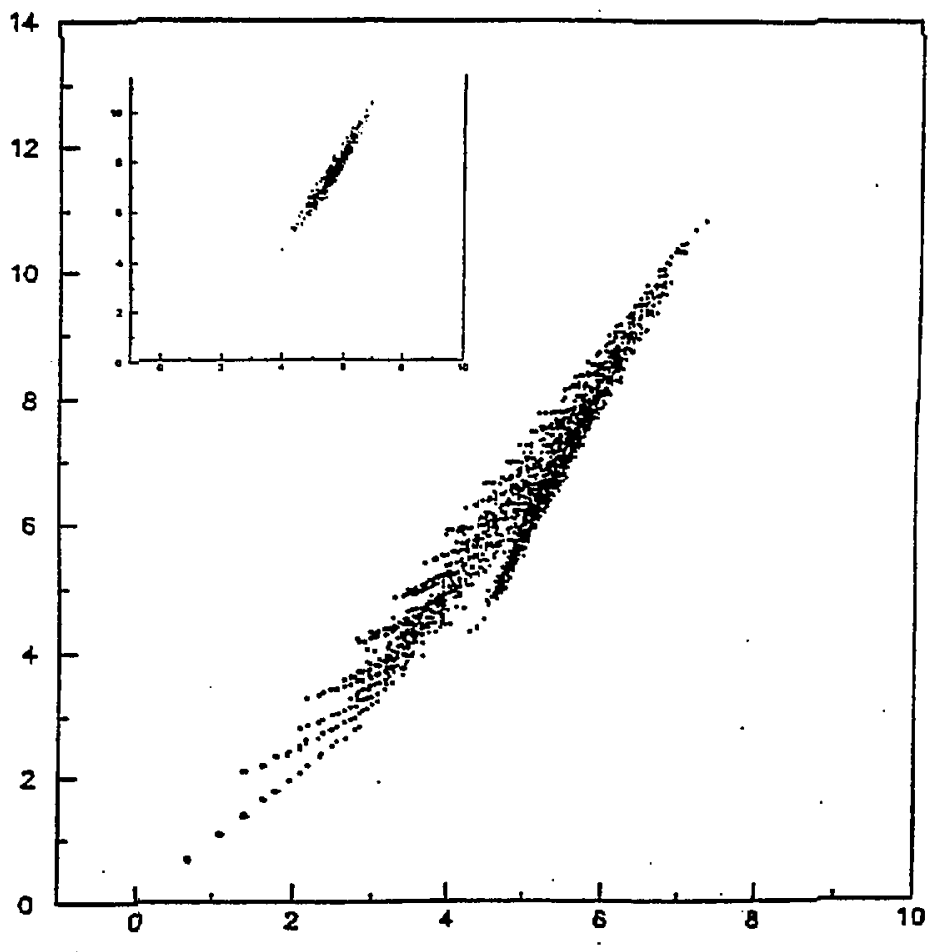

Figure 17: $\ln \left(M_{3}\right)$ versus $\ln \left(M_{2}\right)$ for 25000 event simulation of a 108 nucleon system. 


\begin{tabular}{ccc}
\hline & Percolation & Liquid-gas \\
\hline$\tau$ & 2.19 & 2.21 \\
$\sigma$ & 0.45 & 0.64 \\
\hline
\end{tabular}

Table 2: Predicted critical exponents.

Further insight is given by plotting $M_{2}$ versus the reduced multiplicity $n$. In an infinite system, all $M_{k}$ for $k>\tau-1$ diverge as $n \rightarrow n_{c}$, where $n_{c}$ is the critical reduced multiplicity. Figure 18 shows percolation simulations of $\ln \left(M_{2}\right)$ vs $n$ for various size systems ranging from 27 to 125,000 constituents. The approach to singular behavior of $M_{2}$ is clearly seen for the largest system, but a peak is evident for systems of $\sim 100$ constituenis. The critical events are those with the largest values of $\mathrm{M}_{2}$.

A comparison between percolation theory (Fig. 17) and measuremeuts made for the system $1 \mathrm{~A} \mathrm{GeV} \mathrm{Au}+\mathrm{C}$ (Fig. 19) shows a remarkable similarity. However, we need to understand what drives the disassembly of the nucleus in the energy range of 1 to $3 \mathrm{GeV}$ energy deposition.

If, in each event, we find the largest fragment, we can compute the average size $P(n)$ and deviations from this average $\Delta P(n)$ as a function of event multiplicity, $n$. This is a direct demonstration of the critical fluctuations i.e., the smoking gun for a second order phase transition. Campi has shown that the deviations $\Delta P(n)$, computed from the $1 \mathrm{~A} \mathrm{GeV} \mathrm{Au} \mathrm{data,} \mathrm{peak} \mathrm{at} \mathrm{the} \mathrm{critical} n_{\mathrm{c}}$ as predicted by a 3-dimensional percolation simulation (see Fig. 20) $[53,54,55,56]$. A careful measurement of the location and maximum value of $\Delta P(n)$ will also allow determination of $\beta$ where $\beta=(\tau-2) / \sigma$.

The predictiuns of the percolation $[58,59]$ and liquid-gas [60] phase transition for $\tau$ and $\sigma$ are shown in Table 2.

The comparison of exclusive multifragmentation data with the percolation picture is a necessary, but not a sufficient, step to fully understand critical phenomena. What is missing are the temperature and density information i.e., the critical indices describe the fluctuations but do not identify the driving parameters. In tiny systems the temperature is not a well defined number as is indicated by the breadth of the $M_{2}$ peak. This fact has long been misunderstood in nuclear physics. However, the energy deposition which disassembles the nucleus and contributes to the freeze out kinetic energy must be mea. sured. That is, the transverse energy distribution of the associated protons with a slope parameter of $\sim 8 \mathrm{MeV}$ must be determined on an event by event basis. Further, the freeze out radius can be measured using two particle correlations. Both of these quantities can be measured using the EOS TPC, TOF, and MUSIC detectors. A high resolution downstream detector should be added to aid in connecting tracks between the TPC and the downstream TOF and MUSIC detectors. Because the multifragmentation reaction is fully developed at $5 \mathrm{~A} \mathrm{GeV}$, this is the highest energy required for these measurements. 


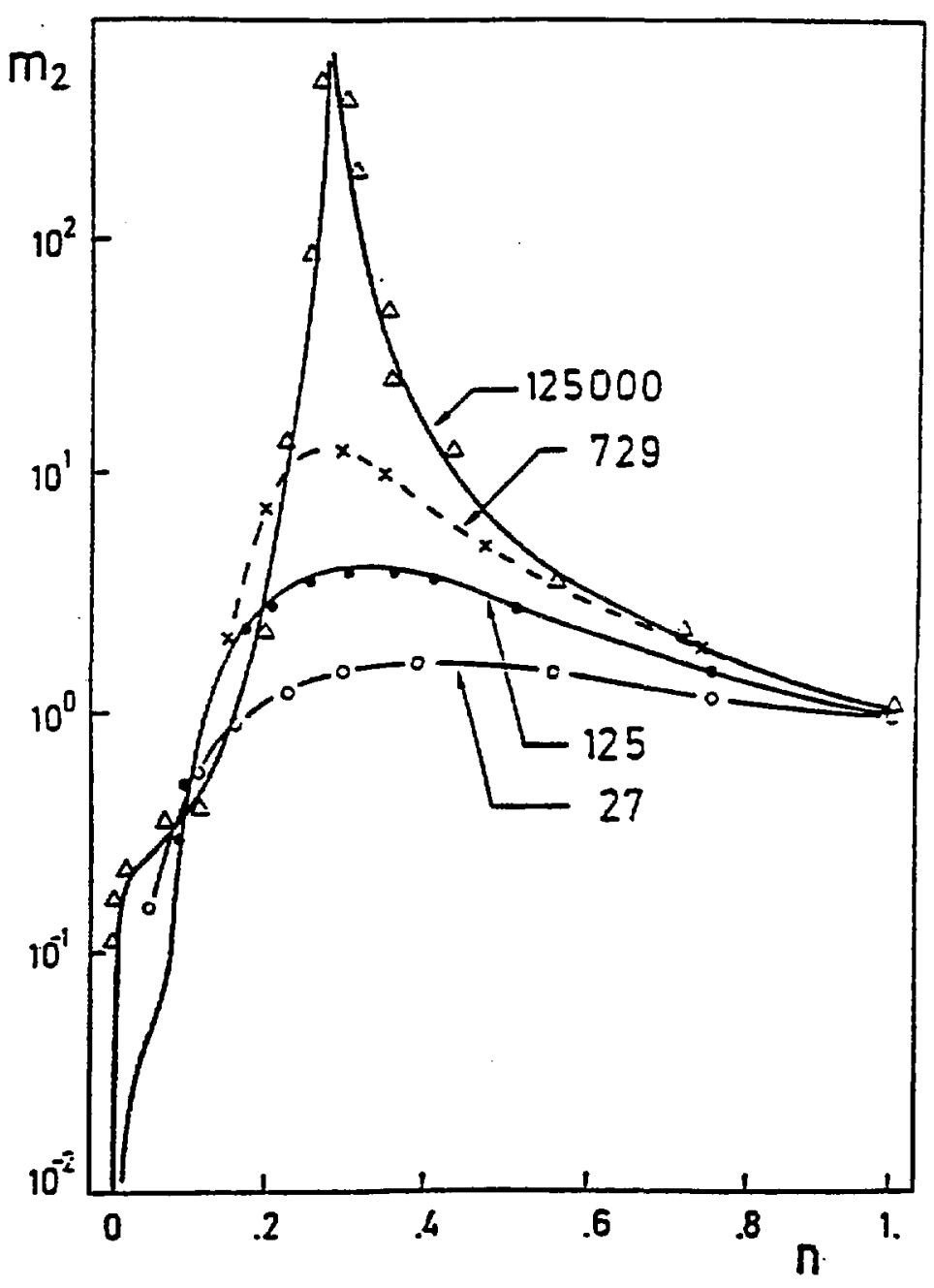

Figure 18: Percolation simulation predictions of $\ln \left(M_{2}\right)$ versus $n$ for systems of different sizes. 


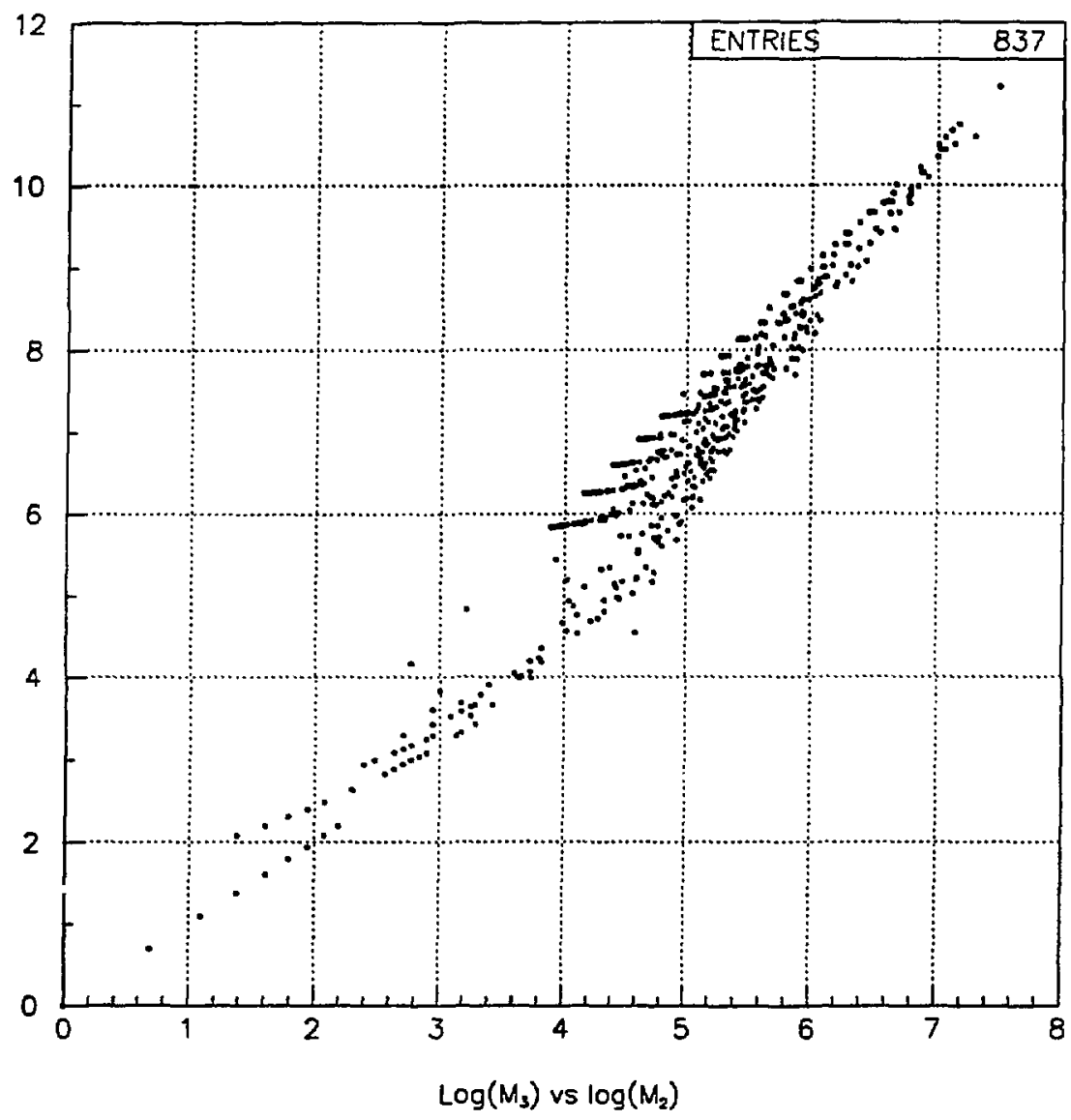

Figure 19: Preliminary data analysis of $1.0 \mathrm{~A} \mathrm{GeV} \mathrm{Au}+\mathrm{C} \operatorname{EOS}$ data for $\log \left(M_{3}\right)$ versus $\log \left(M_{2}\right)$. 

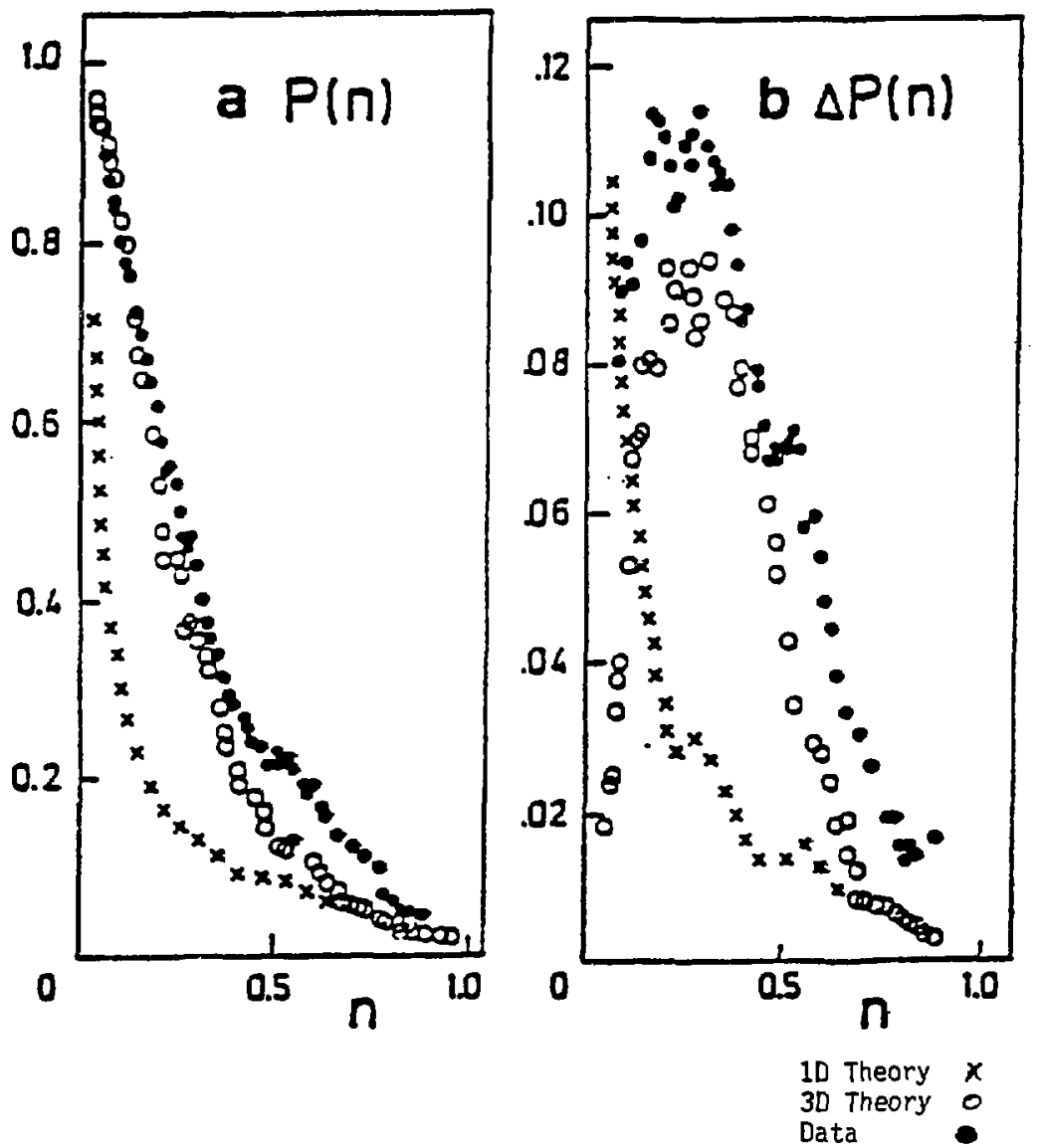

Figure 20: Average size of the largest fragment and fluctuations. Percolation simulations and data are for 197 constituents. 


\section{Experimental Configuration}

The aim of this experiment is to reconstruct, in its finest detail possible, the collision between two heavy ions with an emphasis placed on the exclusive measurement of the most abundantly produced light mass particles (e.g., $\pi, \mathrm{p}, \mathrm{d}, \mathrm{t},{ }^{3} \mathrm{He},{ }^{4} \mathrm{He}$ ), heavy nuclear fragments, strangeness $\left(\Lambda, K^{ \pm}, K_{i}^{0}, \Xi-, \Omega^{-}\right)$and anti-proton production.

Individual event characterization is a very important part of the physics program pertaining to collective effects in hot, dense nuclear matter and is absolutely essential for the study of critical phenomena in nuclei. Ideally, one wants to obtain a complete picture of the four-momenta of all the emitted particles.

The scientific objectives of this proposal can be accomplished with a set of detectors available from LBL. Our apparatus will provide substantial phase space coverage in the center of mass system with the capacity for simultaneous particle identification. The principal detector element is called the EOS TPC which does the bulk of the charged particle tracking. Recently, these detectors have been used successfully in a series of experiments at the Bevalac. A similar arrangement at the AGS is sketched in Fig. 21 and comprises:

EOS TPC: A large volume Time Projection Chamber provides three dinensional tracking of charged particles covering almost $4 \pi$ solid angle in the center-of-mass frame (angles less than $\sim 75^{\circ}$ in the laboratory). Particle identification is obtained from the magnetic rigidity and multiple energy loss measurements.

MUSIC: A large volume, multiple sampling ionization chamber measures the trajectory and identifies the charge of heavy fragments ranging from Carbon to beam particles.

TOF WALL: A movable array of scintillator slats measure the time of flight of light mass fragments and identifies their element number.

MUFFINS: A scintillator based spectrometer detects neutrons, its energy and angle of emission.

TRIGGER: Beam diagnostic and trigger counters complete the basic system

MSGC TPC: A Micro-strip gas counter (MSGC) readout TPC provides high resolution tracking downstream of the EOS TPC.

SDC: Silicon Drift Chamber measures and identifies secondary decays clase to the target.

The figure shows the EOS TPC located between the pole tips of a large apert ure dipole magnet, which should be consifered symbolic of the MPS magnet or a different magnet in another beam line. In combination with the EOS TPC, the MUSIC detector and behind it the TOF wall, are used to identify the forward going projectile fragments which are beyond the dynamic range of the TPC. These two detectors provide full acceptance for the reverse binematic experiments, for example, Au beam on a hydrogen target.

The detectors are modular in nature and are easily transported around the AGS floor. MUSIC, TOF, and MUFFINS each have their own carts and require no major external 


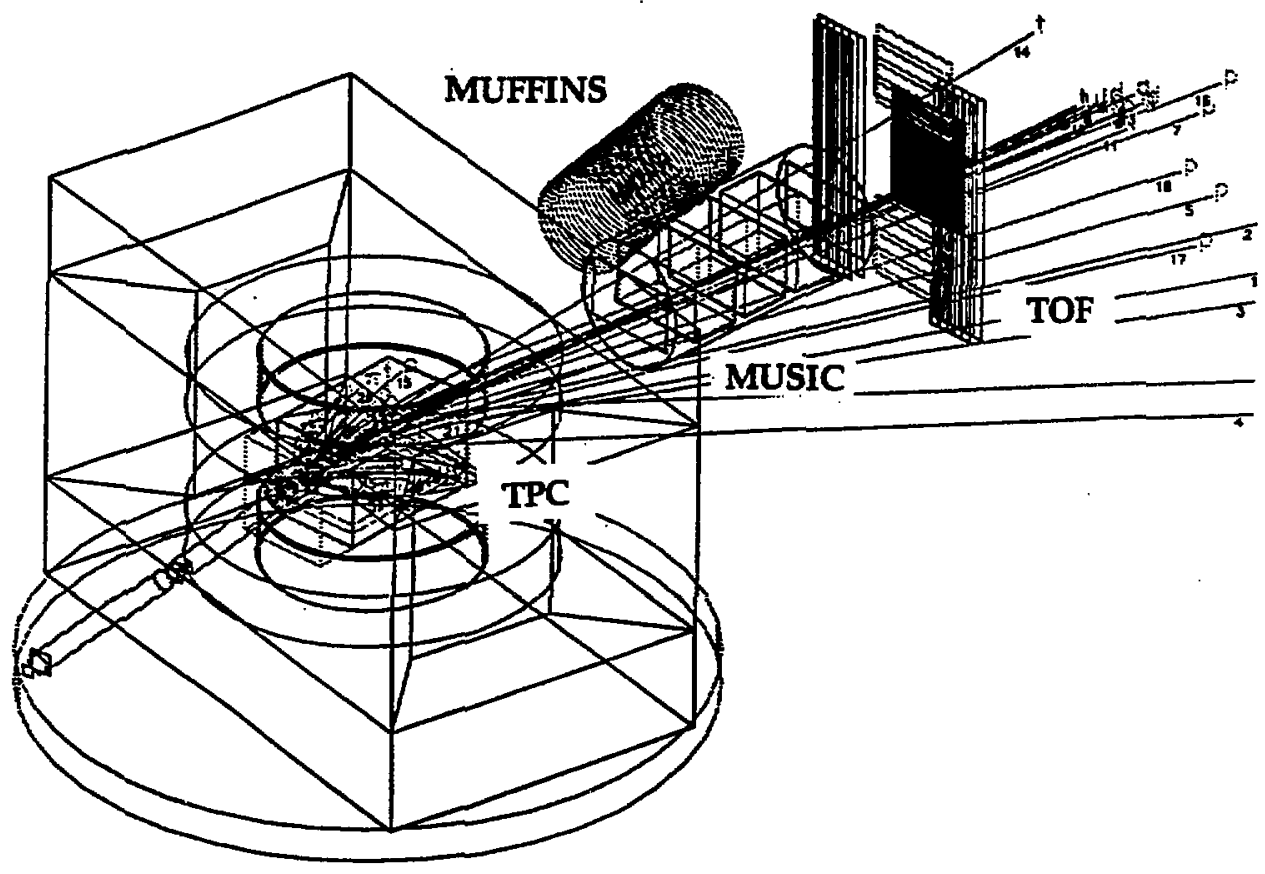

Figure 21: Proposed set up for the EOS experiment at the AGS (perspective view). 
support services. The EOS TPC comes complete with its own hydraulic trolley and auxiliary service hardware, such as gas handling and power supply systems.

The MSGC TPC and SDC are, at present, advanced detector technologj R\&D projects entering into the prototype development phase. We anticipate using the prototypes in our experiment assuming they are operational at beam time. These detectors will extend the capabilities of the experiment and enrich the information available on an event-by-event basis, which must surely add to the physics program.

The span of beam energy from 2.0 to $10 \mathrm{~A} \mathrm{GeV}$ poses a challenge to provide a constant level of performance considering the mix of detectors used in this experiment. The four major detectors are affected differently as the beam energy increases and in the following sections we address the jssues of capability and acceptance. Finally, we summarize the limitations of the apparatus and its implications.

\subsection{The EOS Time Projection Chamber}

The EOS Time Projection Chamber (TPC) is one of the most ambitious detectors ever built for a nuclear physics experiment, and has become the blueprint for future central detectors of the NA49 experiments at CERN and the STAR TPC experiments at RHIC. In this section we briefly describe the TPC, its operation, and performance capability. Further details can be found elsewhere [61].

The EOS TPC was designed primarily to study central collisions with the most energetic and heaviest beams available at the Bevalac. The TPC provides almost $4 \pi$ solid angle coverage, continuous tracking, momentum measurement, and particle identification all at once. As a continuous tracking detector it has a good capability to resolve highmultiplicity events, including secondary vertices $\left(\Lambda, K_{s}^{\circ}\right)$ and decays in flight $\left(\pi, \mu, K^{ \pm}\right)$. However, its design is such that it covers the participant and some spectator region for the highest energy Au beams available at the AGS. Supplementary detectors, for example the MUSIC and TOF detectors, are added downstream of the TPC to provide acceptance and particle identification for nuclear fragments heavier than $Z=5$.

\subsubsection{Detector Description}

The EOS TPC, sketched in Fig. 22, is constructed in the shape of a single rectangular box and is designed to operate at one atmosphere pressure. It is divided into two distinct regions: a large detection volume enclosed by four field cage panels on the sides and an amplification region directly beneath it consisting of wire planes and a cathode pad plane. The active drift volume is $150 \mathrm{~cm}$ long in the beam direction, $96 \mathrm{~cm}$ wide and $75 \mathrm{~cm}$ high in the drift direction. The detector is encased in a light weight aluminum stin for gas containment and thermal jsolation, and to minimize multiple scattering so that the TPC. can be operated in combination with other detectors. The high voltage plate and the field cage provide a homogeneous $(120 \mathrm{~V} / \mathrm{cm})$ electric field gradient in the vertical direction. The TPC detector fits inside a dipole magnet such that the electric and ragnetic fields are parallel. The momentum of a charged particle is determined from the curvature of the track in the magnetic field.

The pad plane is a single panel with an array of $1.2 \mathrm{~cm} \times 0.8 \mathrm{~cm}$ pads covering a $96 \mathrm{~cm}$ by $150 \mathrm{~cm}$ rectangle $(15,360$ pads total) as depicted in Fig. 23 . The pad array comprises 


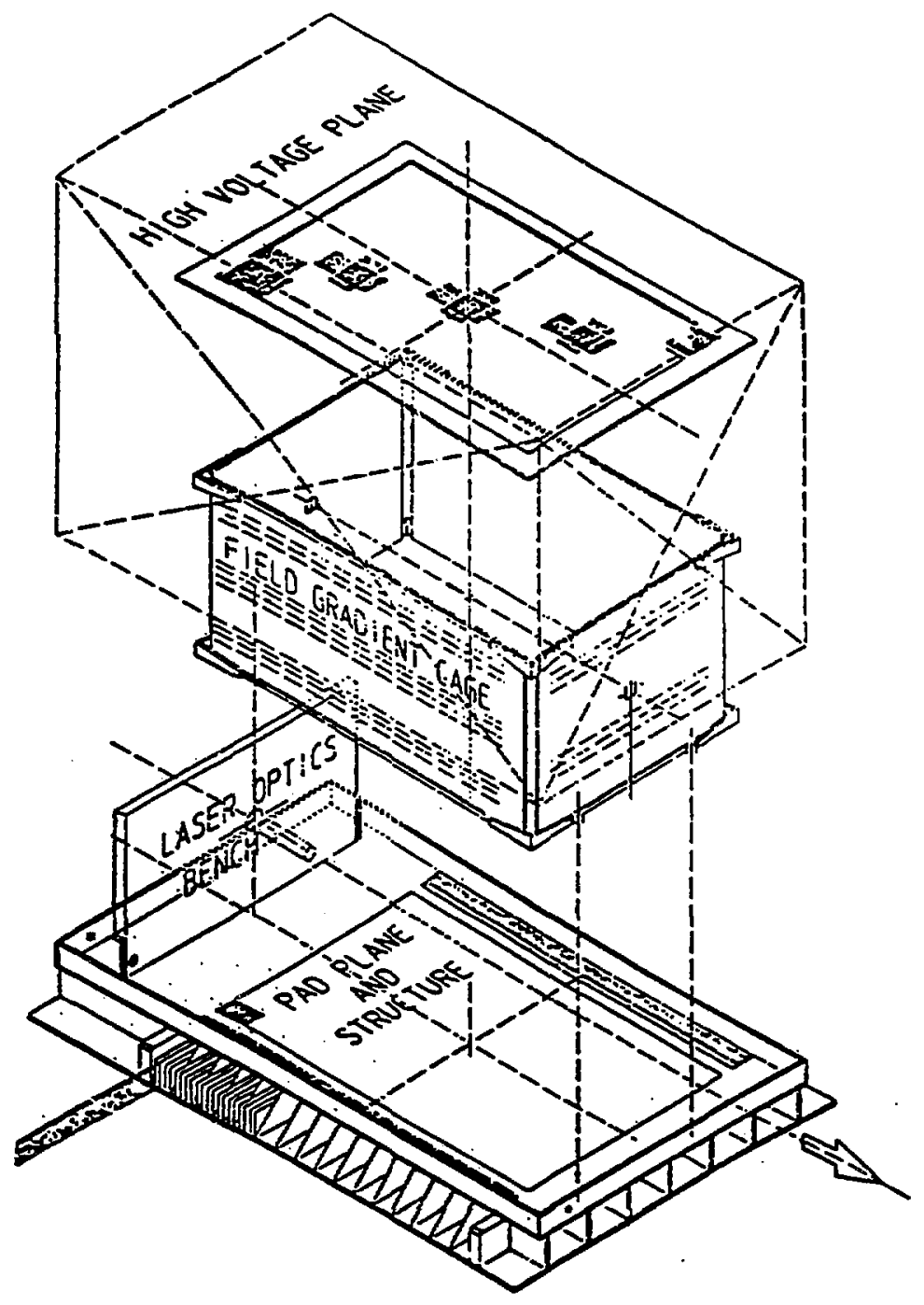

Figure 22: The EOS TPC. 


\begin{tabular}{lr}
\hline Pad Plane Area & $1.5 \mathrm{~m} \times 1.0 \mathrm{~m}$ \\
Number of Pads & 15,360 \\
Pad Size & $12 \mathrm{~mm} \times 8 \mathrm{~mm}$ \\
Drift Distance & $75 \mathrm{~cm}$ \\
Time Sampling Frequency & $10 \mathrm{MHz}$ \\
Flectronic Noise & $700 \mathrm{ems}$ \\
Gas & $90 \% \mathrm{Ar}+10 \% \mathrm{CH}$ \\
Drift Velocity & $5.5 \mathrm{~cm} / \mu \mathrm{s}$ \\
Pressure & 1 Atmosphere \\
B-Field & $13000 \mathrm{Gauss}$ \\
Double .rack Resoiution & $2.5 \mathrm{~cm}$ \\
Solid Angle Coverage & $\leq 2 \pi$ in Lab \\
$d E / d x$ Range & $\mathrm{Z}=1-6$ \\
\hline
\end{tabular}

Table 3: TPC Parameters.

128 rows by 120 columns giving $100 \%$ coverage for complete three dimensional read-out, unlike other conventional TPCs. Above the pad plane are three parallel planes of wires. The first plane consists of an alternating pattern of field and sense wires, the next plane is an isolation grid, and the third plane is called the gating grid.

An incoming charged particle produces ionization electrons along its path through the TPC gas volume. These electrons drift smoothly along the direction of the electric fiedd and are collected on a particular sense wire where they undergo aval anche multiplication. The image pulse appears, by induction, on the cathode pad array. For each segment of track the drift time provides one coordinate, while the induced signals on the pad rows allow the determination of the center-of-gravity of the other two coordinates. Since, the TPC provides up to 128 position and $d E / d x$ sample measurements along each track, it gives good momentum measurement and particle identification. The main parameters of the TPC are summarized in Table 3.

The gating grid acts as a shutter which normally prevents the ionization electrons from reaching the amplification region and, in turn, positive ions generated at the serse plane from escaping into the detection volume. This limits the space charge distortion to a negligible value. The gating grid is fully deactivated $\sim 400$ as after receiving a valid event trigger and remains open for $25 \mu \mathrm{s}$ which is sufficiently long to collect all the electrons in the drift volume.

The TPC sense wires are operated at $\approx 1170 \mathrm{~V}$. With a gas mixture of $90 \%$ Argon and $10 \%$ Methane, a gas multiplication of 3000 is obtained. The sense wires are divided among 16 separate power supplies to allow sections to be operated at reduced gair for analysis of tracks from heavier, more strongly ionizing particles.

The front-end electronics system for 15,360 pads is integrated directly with the TPC detector and uses custom designed, VISI chip technology. The principle advantage of this scheme is the ability to accomplish amplification, shaping, waveform storage, a bigh degree of multiplexing, and digitization immediately below the pad plane. This reduces the 


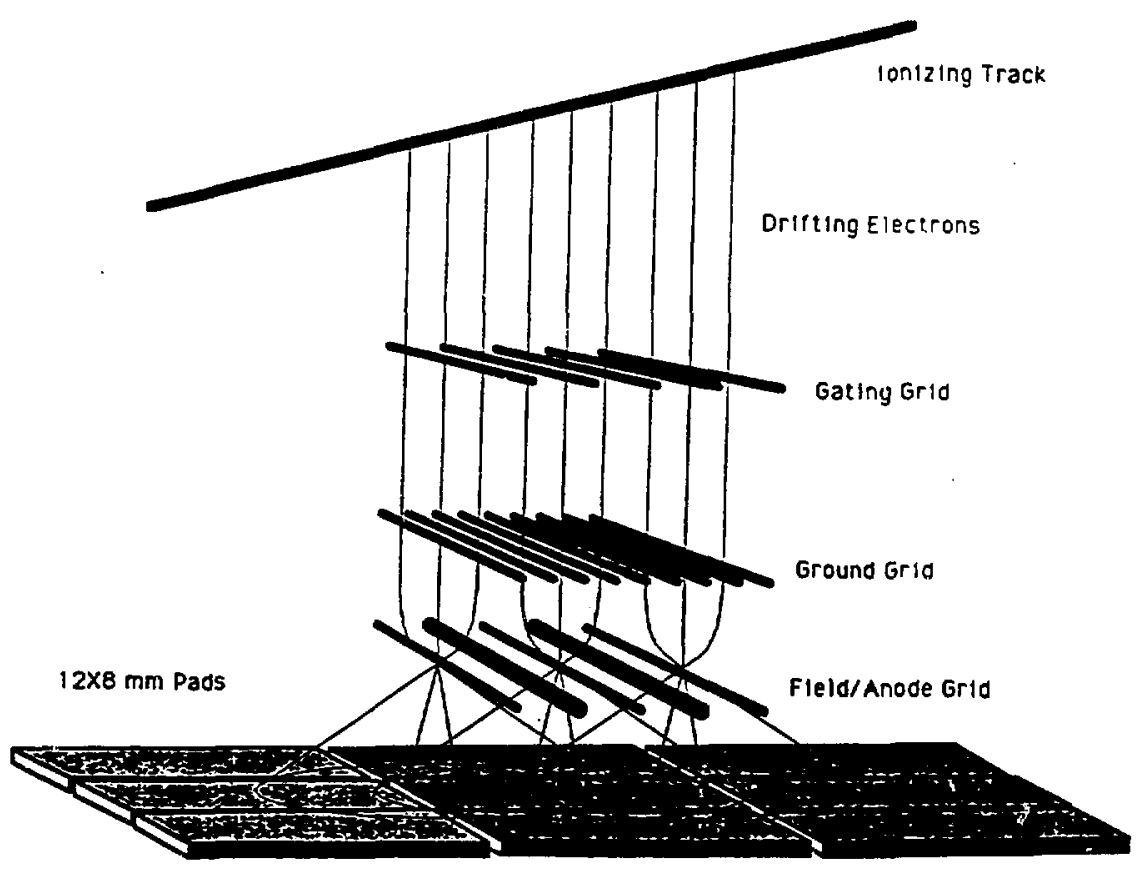

Figure 23: The pad plane of the EOS TPC. 
cabling problerns, and provides substantial noise immunity from extraneous sources. The digital information is transmitted over 128 fiber optic cables and is recejved at a remote site by four VME crates which are all contained in one rack. The data acquisition, tetector configuration, monitoring, and control systems are essentially autonomous systems occupying two racks worth of space in the counting house. The EOS TPC event builder assembles the information from the TPC together with data from all ancillary detectors and sends the event package to be recorded on one of three parallel Exabyte tape units (it may be possible to get greater through-put by using an adoitiona' tape unit). At the same time, complete events are buffered into hardware queues. These event queves are accessible online to both the SUN and VAX workstations using the Ethernet transmission protocol to allow on-Lne event displays and analysis.

\subsubsection{Laser Calibration System}

The EOS TPC is equipped with a Laser Calibration System. Its principal functions are to monitor the electron drift velocity in the TPC and to map the static distortions in the electron drift $\mathrm{pa} h$ due to the non-uniformities in the electric and magaetic fields. In practice, the magnetic fringe field overwhelms the small scale aberrations that might exist in the electric field. These distortions are corrected in the space point reconstruction software. The drift vilocity is sensitive to environmental variables such as the gas temperature and pressure. The temperature is controlled within $0.1 \mathrm{C}$ of its nominal setting. However, the TPC operates at atmospheric pressure and long term pressure variations are possible. Therefore, the drift velocity is monitored continuously during the experiment.

The laser calibrat' on system uses a Nd:YAG laser to produce a primary beam which is frequency quadrupled to give a wavelength of $266 \mathrm{~nm}$ (UV). At this frequency, the laser light has sufficient energy to jonize impuritjes in the P10 gas. The majority of the power from the primary beam is dumped and the intensity of the remainder is controlled with a half-wave plate on a I otorized rotation stage followed by a Glan polarizer. The radius of the beam is reduced with a two-lens focusing telescope and the precise pasition and angle is defined by two mirrors on remotely controlled linetic mounts.

The UV beam is transported from the laser ti the TPC and is split into 18 calibration beams on 2 optics board that is mounted vertically on the up-stream end of the TPC. The position of the iicident beam st measured at several location with quadrant silicon detectors to verify th: precise alignment of the beams. Twelve of the calibration beams are fed through the electric field cage and into TPC gas volume parallel to the beam 2xis, the remaining six take angled paths that intersect with the parallel beams at several locations. These intersections define points in three dimensions as opposed to the beams themselves which onl: constrain two dimensions.

\subsubsection{Event reconstruction using real data}

In Fig. 24 and Fig. 25 we show a semi-central $\mathrm{Au}+\mathrm{Au}$ collision at $1.0 \mathrm{~A} \mathrm{GeV}$. The data were taken at the Bevalac. The crosses represent the actual space points reconstructed from the pad signals. The solid lines show the trajectories of found and fitted tracks. Each track is number ad and labelled by its particle identification. 


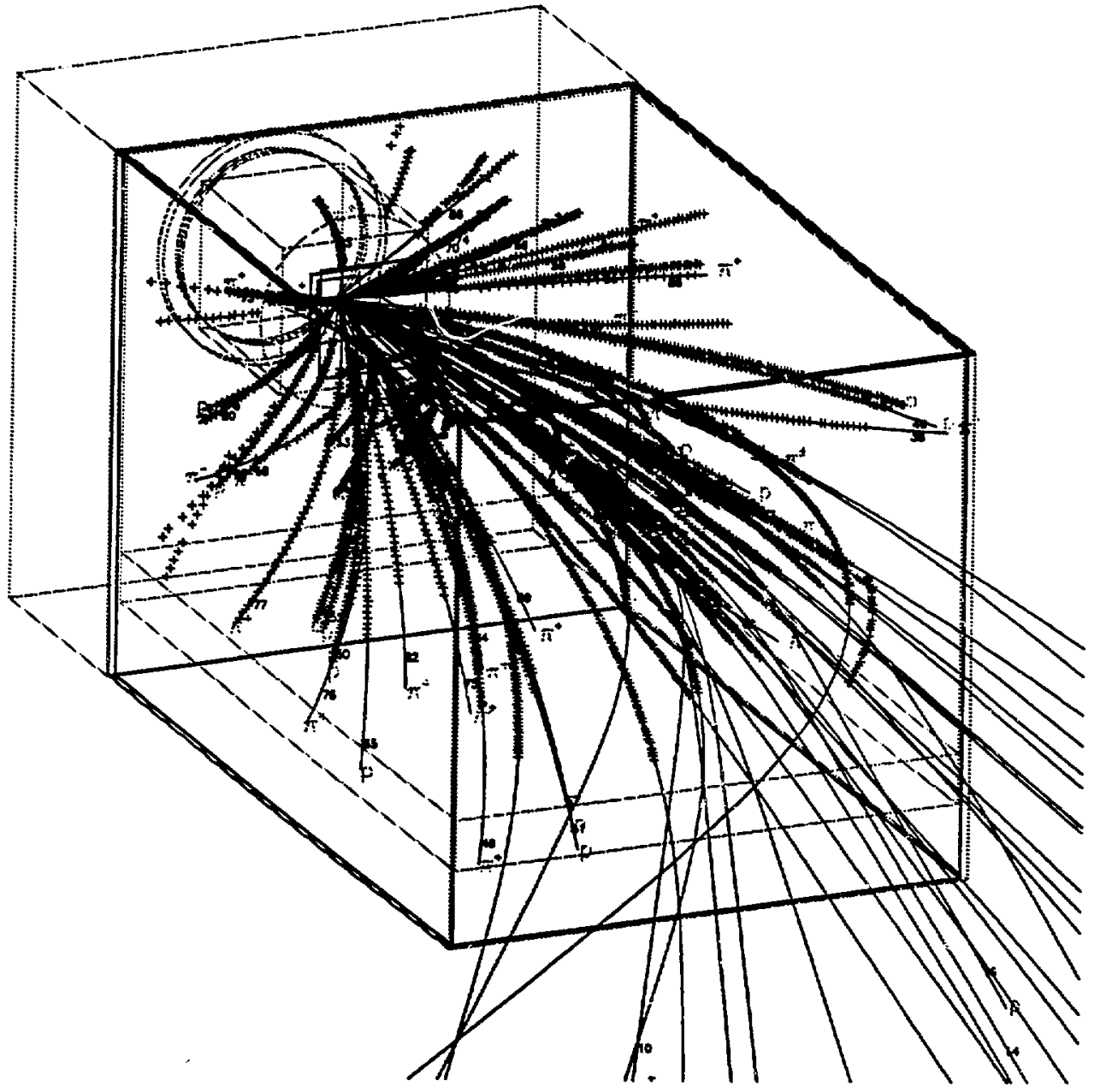

Figure 24: Perspective view of a $\mathrm{An}+\mathrm{An}$ event at $1.0 \mathrm{~A} \mathrm{GeV}$ beam energy. 


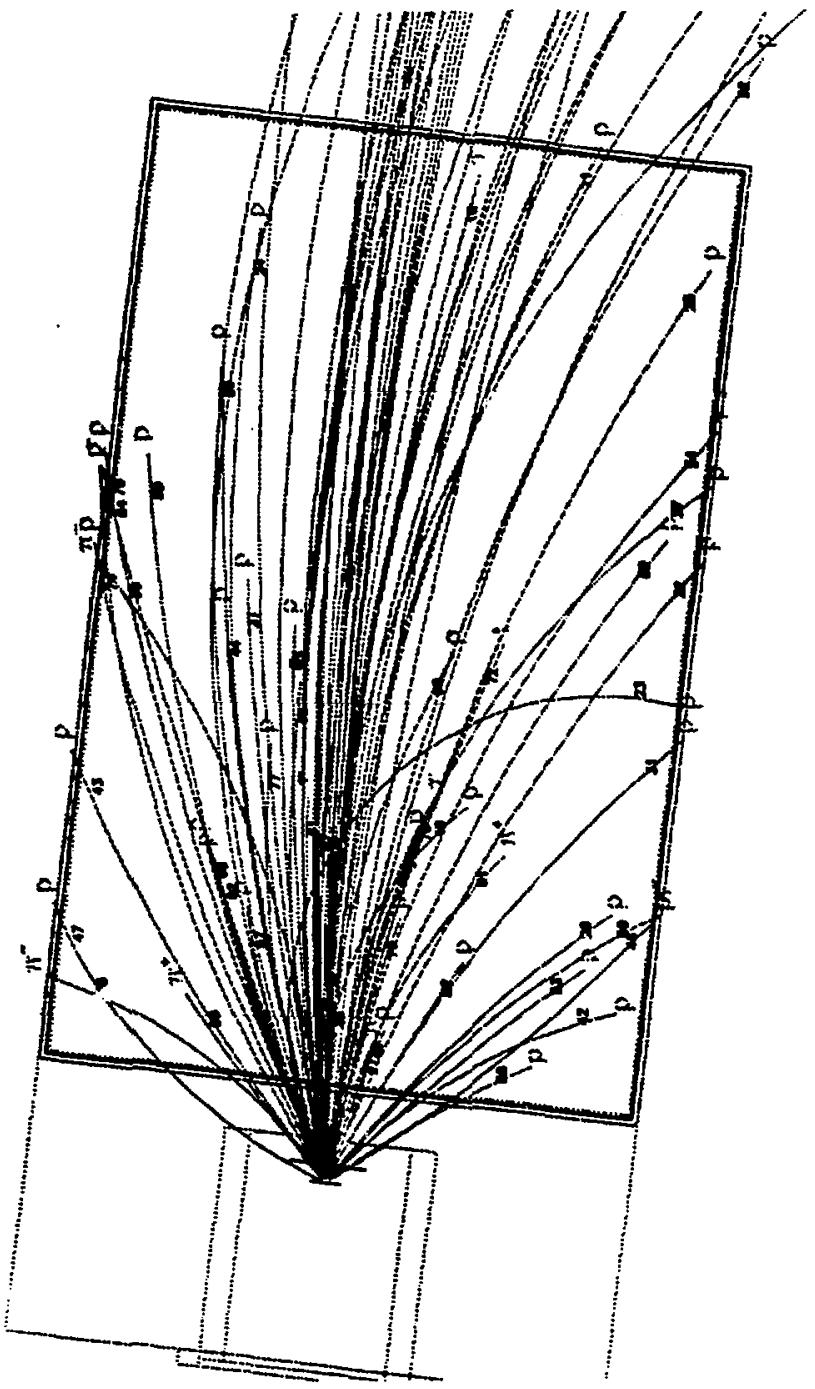

Figure 25: Reconstructed Au + An event projected onto the pad plane. 


\subsubsection{Particle Identification and $d E / d x$ Resolution}

Charge pasticle identification in a TPC is accomplished by multiple sampling of ionization along the trick. The method relies on the fact that the most probable value of ionization energy loss $(d E / d x)_{m p}$ in a sample of gas is a function of the incident particle's velocity rather than it's momentum. Thus a simultaneous measurement of $(d E / d x)_{\text {mp }}$ and momentum per charge $p / Z$ allows particles to be uniquely jdentified over a wide range of momenta.

The dominant factor limiting the $d E / d x$ resolution of a TPC detector is the Landau fluctuation of the energy loss. The large Landau fluctuations (0.5-1.0 times FWHM) in thin gas samples cannot be avoided simply by increasing the thickness of the sample. For particle identification, an appropriate mean value must be calculated. Normally this is done by taking the truncated mean.

Two examples of $d E / d x$ versus $p / Z$ scatter plots obtained from data taken with the EOS TPC detector are shown in Figs. 26 and 27. The experimental data used to co.sstruct these plots were taken with a $1.2 \mathrm{~A} \mathrm{GeV} \mathrm{Au}$ beam incident on a Au target and a $2.0 \mathrm{~A} \mathrm{GeV}$ $\mathrm{Ni}$ beam impinging on a $\mathrm{Cu}$ target. These are the most energetic beams of their type that the Bevalac was able to provide. Each point represents a reconstructed track and the most probable value $(d E / d x)_{m p}$ is determined from the mean of the lowest $70 \%$ of the $d E / d x$ samples. Bands of identified particles for $\pi^{ \pm}, p, d, t$, and light nuclear fragments up to $Z=7$ are clearly visible. The solid lines show the predicted erergy loss curves while the dashed lines sketch the boundaries assigned to a given particle type.

Figures 28 and 29 show $d E / d x$ versus $p / Z$ for $A R C$ model[11] Au + Au central events at almost maximum AGS energy and about half maximum energy. These events were processed through our full simulation chain, as described below. About $79 \%$ of all reconstructed tracks have 25 or more usable $d E / d x$ samples (corresponding to the upper panels in figures 6 and 7 ), and 2 bout $41 \%$ have 50 or more usable $d E / d x$ samples (corresponding to the lower panels). The simulated resolution as a function of the number of samples is approximately consistent with the experimentally-observed resolution in the EOS TPC at the Bevalac, which achieves $\sigma_{d E / d x} \approx 8 \%$ for minimum ionizing particles traversing close to the full length of the chamber. This level of performance is adequate for separating particles in the $1 / \beta^{2}$ region, but not in the relativistic rise region. This implies that it is feasible to reliably separate deuterons from protons near and below $2.2 \mathrm{GeV} / c$, protons from pions, near and below $1.2 \mathrm{GeV} / c$, and kans from protons below $0.7 \mathrm{GeV} / c$.

It is instructive to quantify the simulated particle identification capabilities of the TPC in the specific context of the physics goals of the proposed experiment. For studies of : ideward flow, it is necessary only to separate protons from mesons over the rapidity ranige of interest. We emphasize that even for much lighter systems than Au + Au, measurement of the flow signature over a specific rapidity range does not require the use of any information from rapidities outside that range [62]. Sideward flow peaks at projectile and target rapidities, and approaches zero at mid-rapidity. For mass-symmetric systerrs sike Au + Au, good baryon identification at target (or else projectile) rapidities is the onl $y$ essential capability, and this is easily achievable. As we move from target rapidity towards mid-rapidity, misidentification among our proton sample increases. However, our simulations indicate that the majority of the particles above mid-rapidity are protons and 
$\mathrm{Au}+\mathrm{Au} 1200 \mathrm{MeV} / \mathrm{n}$

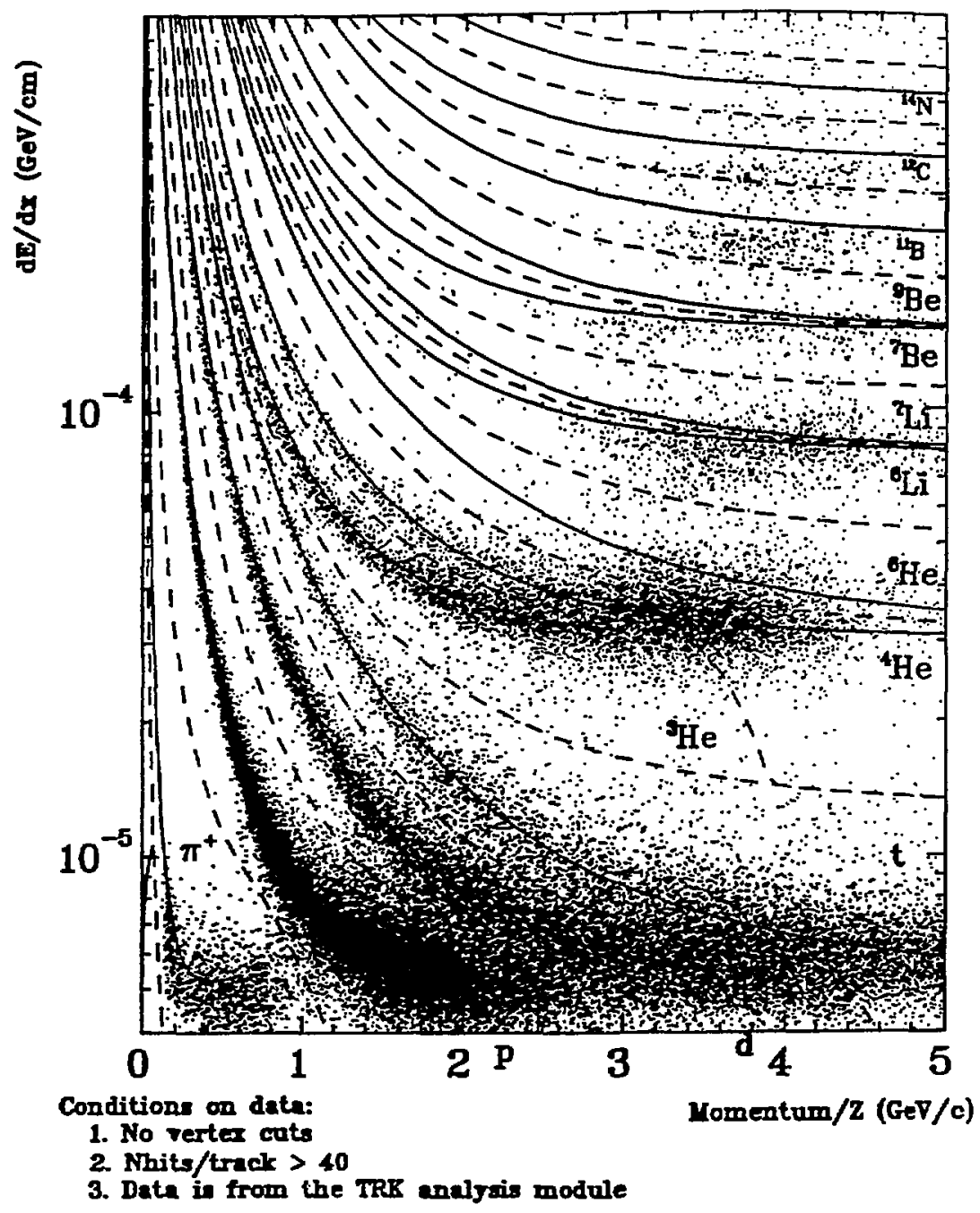

Figure 26: Scatter plot of $d E / d z$ vs rigidity for particles in the Bevalac experiment (Au $+\mathrm{Au}$ ). 


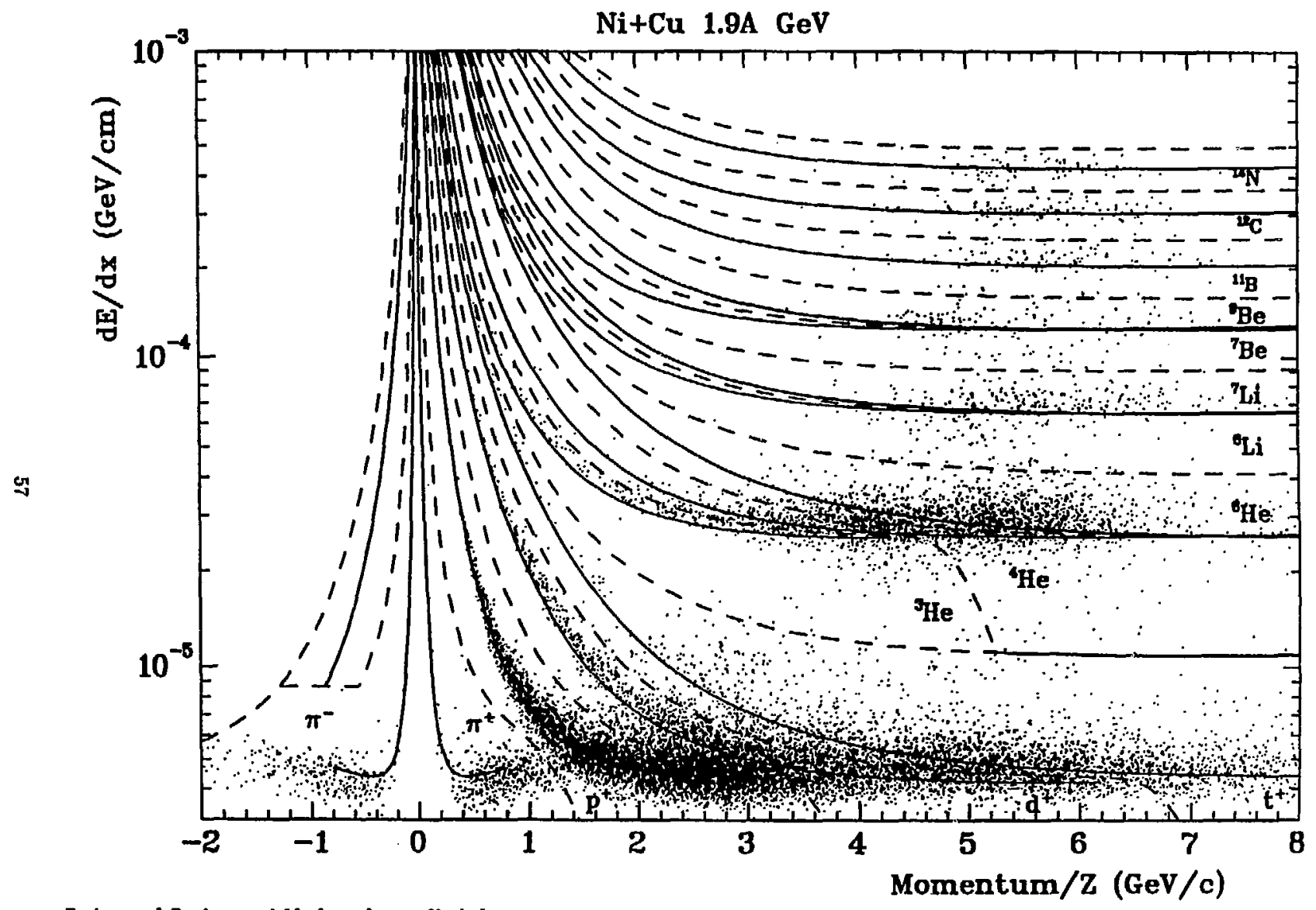

Proton and Dauteron glelds hare been adfanted for ploture olonrity.

Figure 27: Scatter plot of $d E / d x$ vs rigidity for particles in the Bevalac experiment (Ni + Cu). 


$$
\mathrm{Au}+\mathrm{Au} \quad \mathrm{P}_{\mathrm{BEAM}}=8.9 \mathrm{~A} \mathrm{GeV} / \mathrm{c}
$$
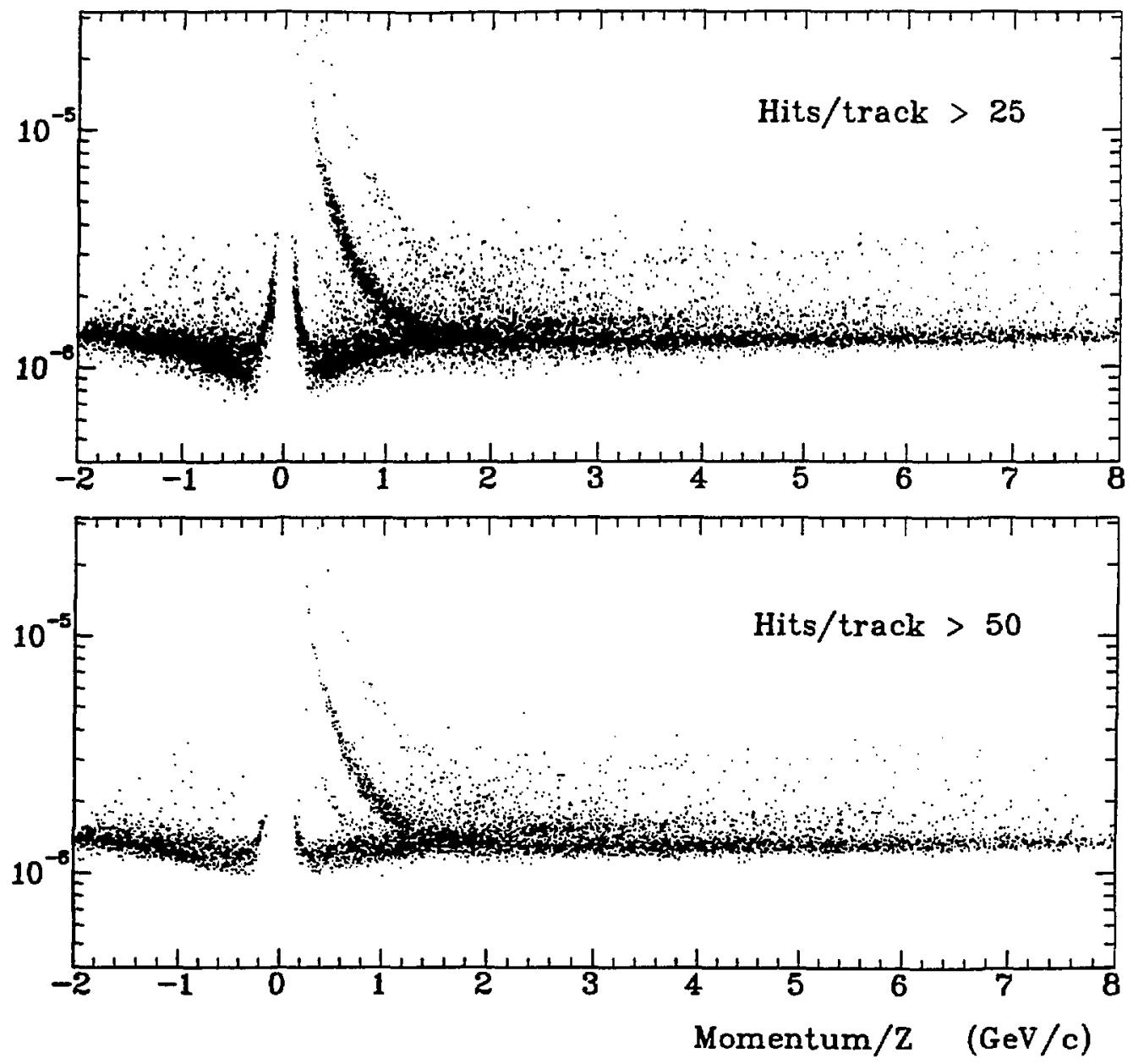

Figure 28: Scatter plot of $d E / d z$ ve $p / Z$ for $8.9 \mathrm{~A} \mathrm{GeV} / c \mathrm{Au}+\mathrm{Au}$ ARC simulations. 

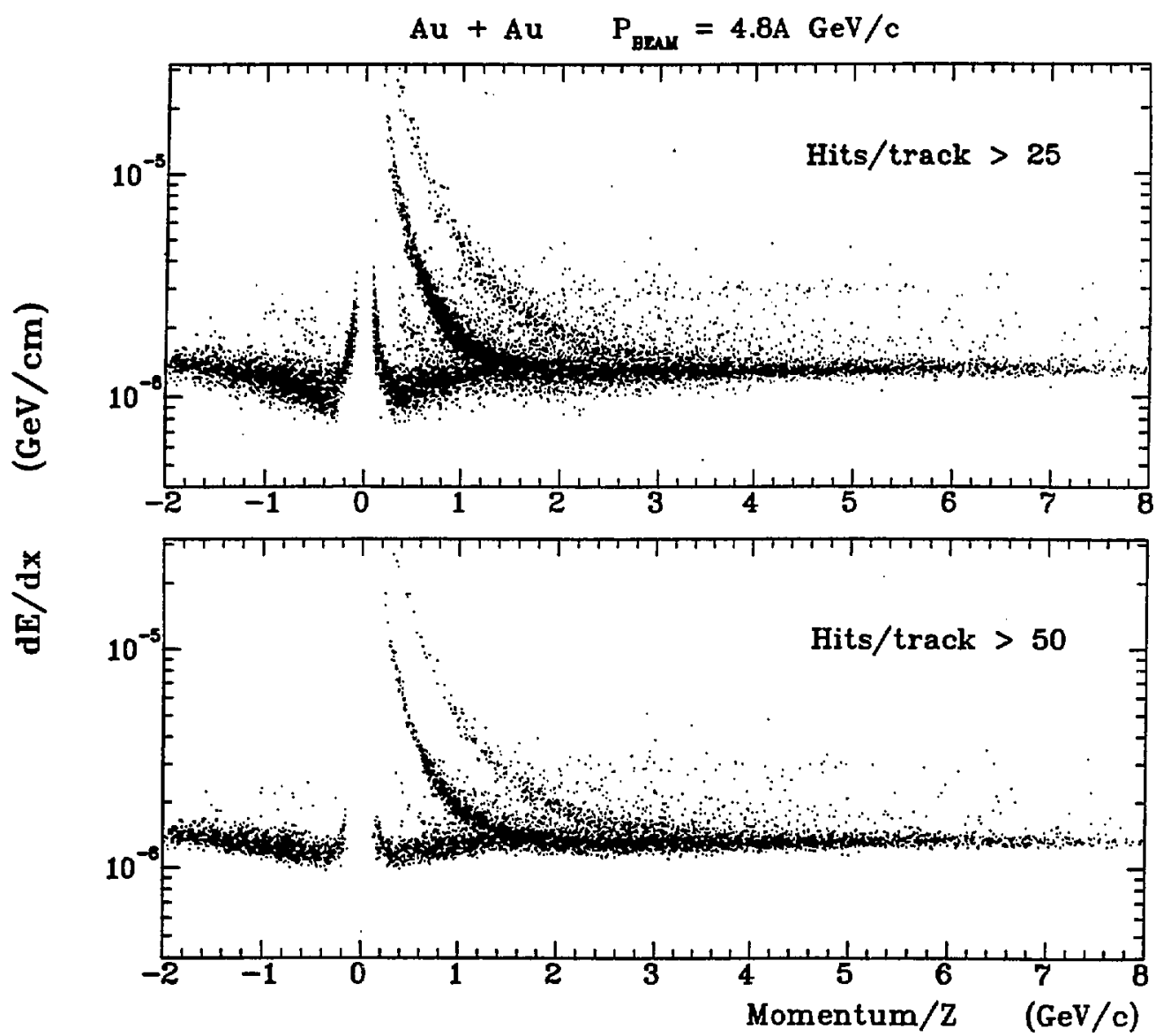

Figure 29: Scatter plot of $d E / d x$ vs $p / Z$ for $4.8 \mathrm{~A} \mathrm{GeV} / \mathrm{c} \mathrm{Au}+\mathrm{Au} A R C$ simulations. 
the level of contamination from $\pi^{+'}$ may be acceptable.

\subsection{Simulations}

Monte Carlo simulations are under way in order to optimize the experimental configuration, to test the resolving power, and to establish the performance of the EOS TPC in central $\mathrm{Au}+\mathrm{Au}$ collisions with beam energy covering the 2-10 A GeV range. We have chosen impact parameter $b \leq 1 \mathrm{fm}$ collicions because they represent the highest multiplicity events that could occur.

The simulation chain begins with event generation followed by a detailed GEANT simulation of the EOS TPC detector. The final output was treated identically by the TPC analysis software as the real data.

For the event generation stage we used three programs: a) the Relativistic Quantum Molecular Dynamics (RQMD) code, b) the ARC internuclear cascade model, and c) the statistical model FREESCO. The first two calculations (RQMD, ARC) provided events spanning the energy range 2-11 A GeV, while FREESCO was used to provide a reference model at $1 \mathrm{~A} \mathrm{GeV}$. The latter calculation is representative of the Bevalac data and therefore served as a standard against which the performance of the TPC soltware could be judged.

\subsubsection{TPC Space Point Reconstruction}

The full GEANT detector modelling program is part of an integrated software package called LOSGX which also includes the EOS TPC analysis shell (TAS). EOSGX has been specifically designed for simulation studies, and track reconstruction efficiency with cross-references to data tables at several points in the analysis chain.

A simulated event is read in by GEANT which in turn swims each particle through the TPC and other elements of the experimental geometry. The center-of-gravity coordinates of a track as it passes over a TPC pad row and its energy loss at that point are recorded. This information is used by the TPC response module which quickly calculates the final set of TPC Hits (coordinates). The philosophy here is not to simulate the production of ionization in the TPC and its subsequent drift and detection at the pad plaite but rather to use empirical algorithms whose constants are adjusted to duplicate (as best possible) the overall characteristics of the observed hits in the TPC. The quality of an observed hit depends on several factors, such as, the track crossing angle and drift distance. In the response module, a cluster of raw hits (pad row crossings) are merged into one if they are within a certain merging distance and assigned an error based of the degree of merging. The positions of the space points are smeared according to the position resolution of the detector.

The results are summarized in Table 4 for Au + Au central collisions as a function of beam energy. The various columns have the following meaning. TPC space points give the total number of pad row crossings and therefore refers to the potential hit candidates. Some fraction of these candidates will be merged. The third column labeled Merged Hits give the number of hits in the TPC which are a result of merging. In this analysis, the merging distances were set to $\Delta y_{\text {merge }}=2.5 \mathrm{~cm}$ in the drift direction, and $\Delta x_{\text {merge }}=$ $1.0 \mathrm{~cm}$ along the pad row. These limitations are imposed by the longitudinal diffusion for 


\begin{tabular}{llllll}
\hline $\begin{array}{l}\text { Beam Energy } \\
\text { A GeV }\end{array}$ & $\begin{array}{l}\text { TPC Space Points } \\
\text { (row crossings) }\end{array}$ & $\begin{array}{l}\text { Merged Hits } \\
\text { in TPC }\end{array}$ & $\begin{array}{l}\text { Isolated } \\
\text { Hits }\end{array}$ & $\begin{array}{l}\text { Total TPC } \\
\text { hits }\end{array}$ & $\begin{array}{l}\text { Merging } \\
\text { rat, } 0\end{array}$ \\
\hline 10.7 & 51,000 & 6,400 & 16,600 & 23,000 & $28 \%$ \\
8.0 & 36,700 & 4,200 & 15,000 & 19,200 & $22 \%$ \\
6.0 & 31,600 & 3,750 & 14,250 & 18,000 & $21 \%$ \\
4.0 & 22,800 & 2,600 & 12,050 & 14,650 & $18 \%$ \\
2.0 & 15,100 & 1,350 & 9,900 & 11,250 & $12 \%$ \\
1.0 & 11,100 & 1,000 & 8,050 & 9,050 & $11 \%$ \\
10.7 & & & & & \\
& 51,000 & 5,700 & 24,100 & 29,800 & $20 \%$ \\
\hline
\end{tabular}

Table 4: Hit merging in the TPC.

P10 gas and the pad response function, respectively. Hits which required no merging are called Isolated Hits. The sum of merged and isolated hits is the number of (useable) Hits in the TPC (fifth column). The last column is the ratio of merged to total hits.

At $1 \mathrm{~A} \mathrm{GeV}$, there are a total of $\sim 9000$ hits per event of which $\sim 8000$ are unmerged, clean hits. The merging ratio is $11 \%$. At $10.7 \mathrm{~A} \mathrm{GeV}$, the total number of hits per event and the merging ratio increased by a factor of $\sim 2.5$ while the potential hit candidates went up from 11,100 to 51,000 , that is by a factor $\sim 4.5$. This implies that more multi-cluster $(\geq 2)$ hits are merged into one.

We have repeated the calculation at $10.7 \mathrm{~A} \mathrm{GeV}$ using a smaller merging distance $\Delta y_{\text {merge }}=1.25 \mathrm{~cm}$ to check what improvement can be made using a gas with low diffusion. The results appear in the last row of the table. The merging ratio decreased by $\sim 8 \%$ to $20 \%$. The number of merged hits fell by 700 while gaining 7500 isolated hits.

The events at 10.7 A GeV appear 2.5 times as dense as a Bevalac event in the EOS TPC. As far as pixel occupancy ( 28-33\%) is concerned this is not a serious concern. However, we are in the process of double checking these results using a fine detail TPC simulation program which produces output in the form of electronic data rather than hits.

\subsubsection{Track Reconstruction Efficiency}

The hits from the fast simulator described above are input to the EOS TPC tracking code. This code is based on conventional algorithms and Kalman filter techniques. The results are given in Table 5. The second column indicates the total number of charged tracks produced in the collision. Next we define the number of tracks in the TPC as those which satisfy the requirement that at least 20 hits belong to the track. The number of good reconstructed tracks is listed in the fourth column. These tracks bad at least 20 hits, and at least $90 \%$ of the hits originated from the same GEANT track. The tracking efficiency is defined as the ratio of found tracks to the number of candidate tracks in the TPC.

The first observation is the number of tracks in the TPC is $\sim 465$ at $10.7 \mathrm{~A} \mathrm{GeV}$, a factor of 3.5 larger than at $1.0 \mathrm{~A} \mathrm{GeV}$. The tracking efficiency $(\sim 90-95 \%)$ is optimum in the $\sim 1-5 \mathrm{~A} \mathrm{GeV}$ energy range and deteriorates to $\sim 65 \%$ at $10.7 \mathrm{~A} \mathrm{GeV}$. A $10 \%$ 


\begin{tabular}{lllll}
\hline $\begin{array}{l}\text { Beam Energy } \\
\text { A GeV }\end{array}$ & $\begin{array}{l}\text { Tracks in } \\
\text { Event }\end{array}$ & $\begin{array}{l}\text { GEANT Tracks } \\
\text { in the TPC }\end{array}$ & $\begin{array}{l}\text { Reconstructed } \\
\text { Tracks }\end{array}$ & $\begin{array}{l}\text { Tracking } \\
\text { Efficiency }\end{array}$ \\
\hline 10.7 & 612 & 465 & 300 & $65 \%$ \\
8.0 & 476 & 362 & 283 & $78 \%$ \\
6.0 & 418 & 327 & 262 & $80 \%$ \\
4.0 & 328 & 259 & 229 & $88 \%$ \\
2.0 & 248 & 178 & 169 & $95 \%$ \\
1.0 & 180 & 131 & 124 & $95 \%$ \\
10.7 & 612 & 502 & 380 & $75 \%$ \\
\hline
\end{tabular}

Table 5: Tracks in the TPC.

improvement on this value is possible by using a cool gas as indicated in the last row. We point out that no attempt has been made to optimize the parameters in the track finding code for high energies.

\subsubsection{Acceptance}

The acceptance of the TPC has been determined using approximately $100 \mathrm{Au}+\mathrm{Au}$ RQMD events at beam energies of 6 and $10 \mathrm{~A} \mathrm{GeV}$. We show three sets of figures at $6 \mathrm{~A} \mathrm{GeV}$ beam energy in Figs. 30 and 31 . The transverse momentum $p_{t}$ versus rapidity $y_{c m}$ scatter plots for the generated events are shown separately for the protons (upper left) and the pions "iower left). The corresponding pictures on the right hand side are distributions for tr iss "accepted" in the TPC, that is, they were deemed good candidates for tri k $k$ finding The difference between "generated" and "accepted" plots defines the geometrical acceptance of the TPC. The rapidity of the beam is 1.6 in the center of mass system. The proton geometrical acceptance is very good and essentially provides full coverage in the interval $-1<y_{\mathrm{cm}}<+1$. The pion geometrical acceptance extends over a large asymmetrical region $-0.5<y_{c m}<+2.0$. The loss near target rapidity is be expected since soft pions will not have sufficient rigidity to reach the TPC. In our simulations we have not attempted to optimize the target position and the relative rotation of the TPC and some improvements might be possible. In Fig. 31 we show similar plots but make the comparison between accepted and found (reconstructed) tracks. This set of pictures show the overall acceptance of the TPC taling into account tracking efficiency. The distributions look very similar which indicates the events are generally fully reconstructed and do not distort the geometrical acceptance. However, for protons there are some track losses in the high rapidity and low $p_{t}$ region but this is of minor importance and might improve if one uses a suitable low diffusion gas. The average reconstruction efficiency is $82 \%$. This value was obtained by taling the ratio of entries in the found and accepted scatter plots. These plots are reproduced in Figs. 32 and 33 but this time for $\mathrm{Au}+\mathrm{Au}$ at $10 \mathrm{~A} \mathrm{GeV}$. 


\section{$A u+A u$ at $6 \mathrm{GeV} R Q M D$}
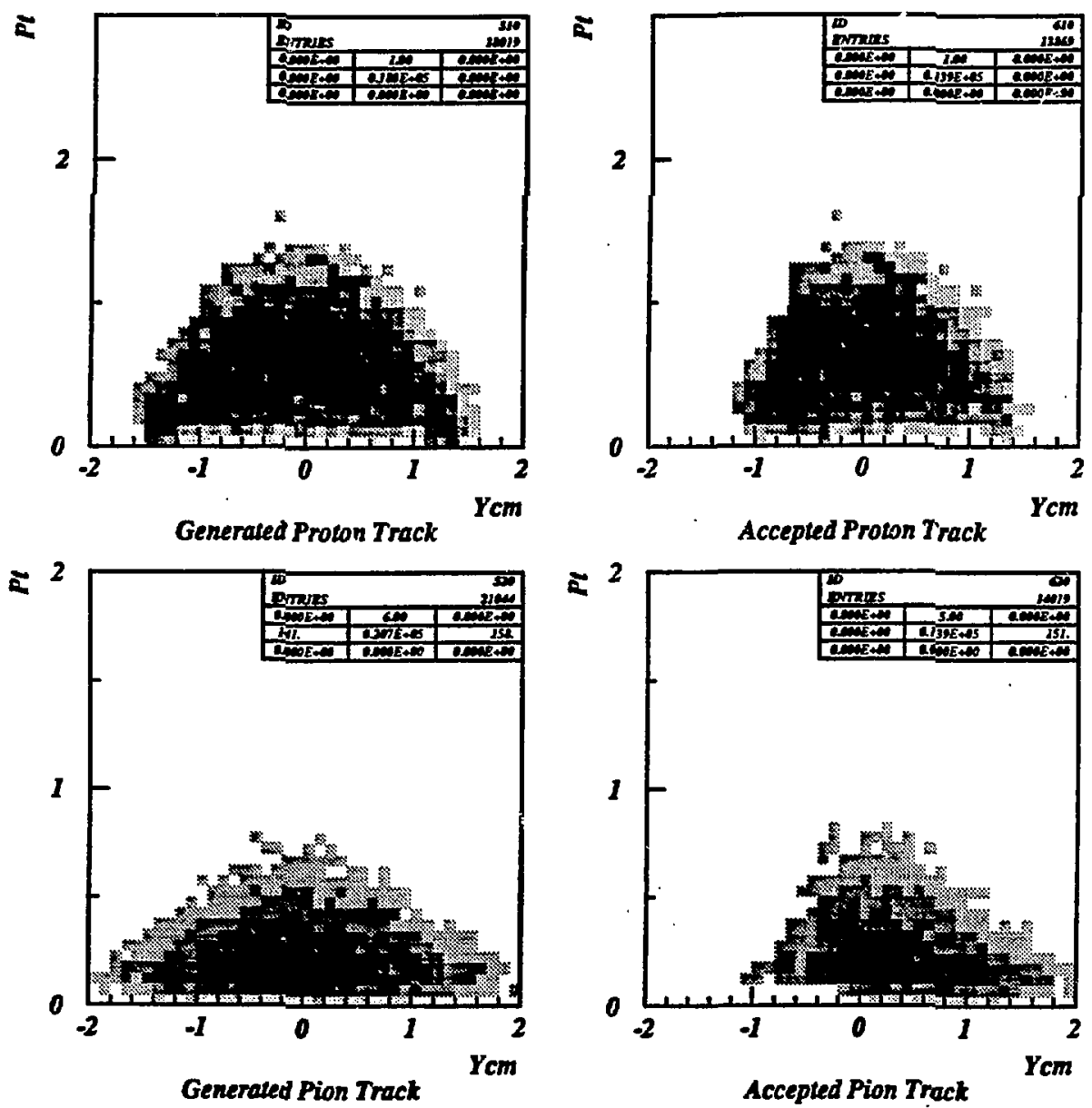

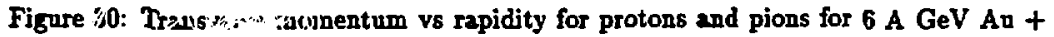
Au RRMP sim." 


\section{$A u+A u$ at $6 \mathrm{GeV} R Q M D$}
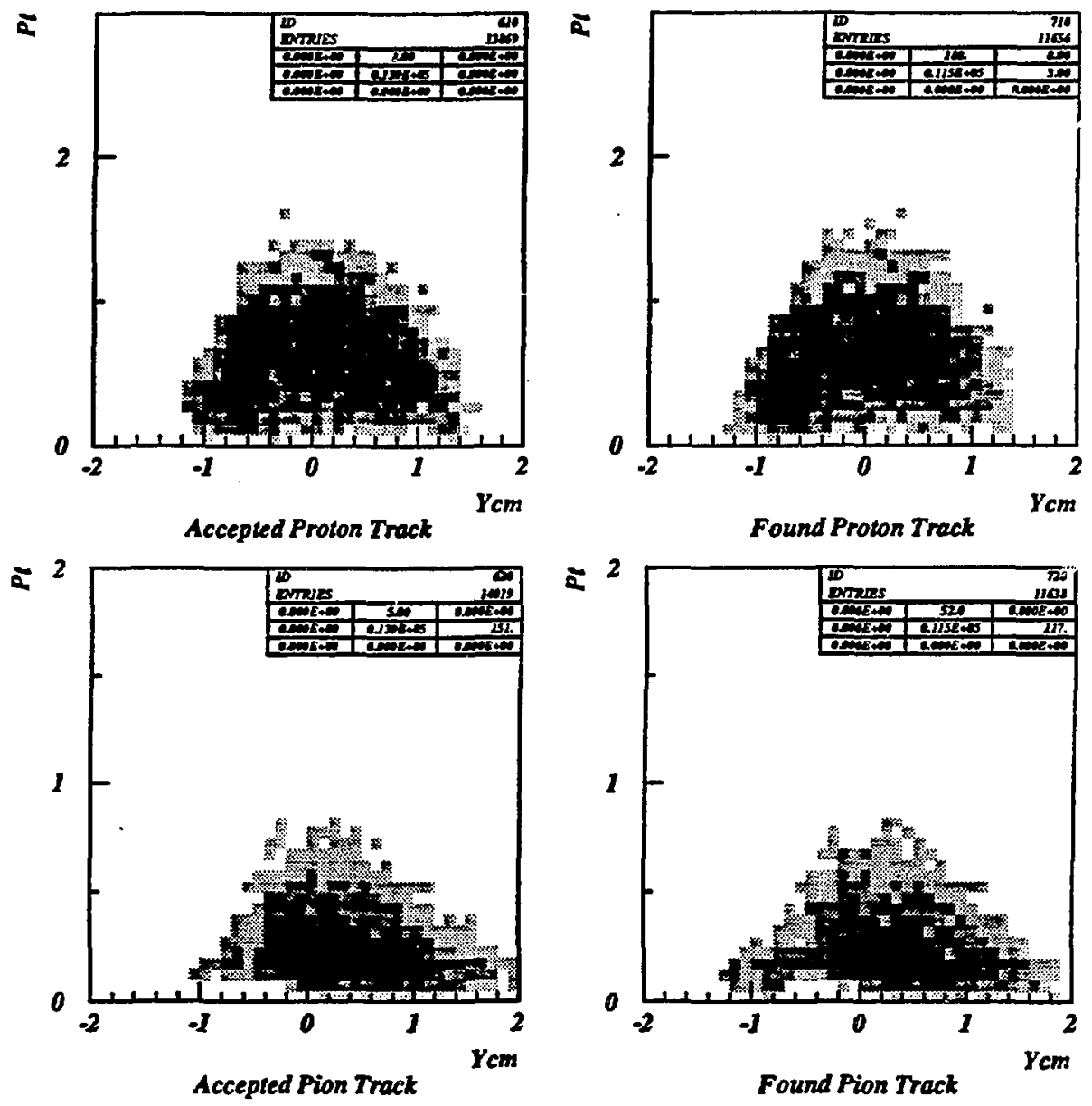

Figure 31: Transverse momentum vs rapidity for accepted protons and pions for $6 \mathrm{~A} \mathrm{GeV}$ Au + Au RQMD simulations. 


\section{$A u+A u$ at $10.7 \mathrm{GeV} R Q M D$}
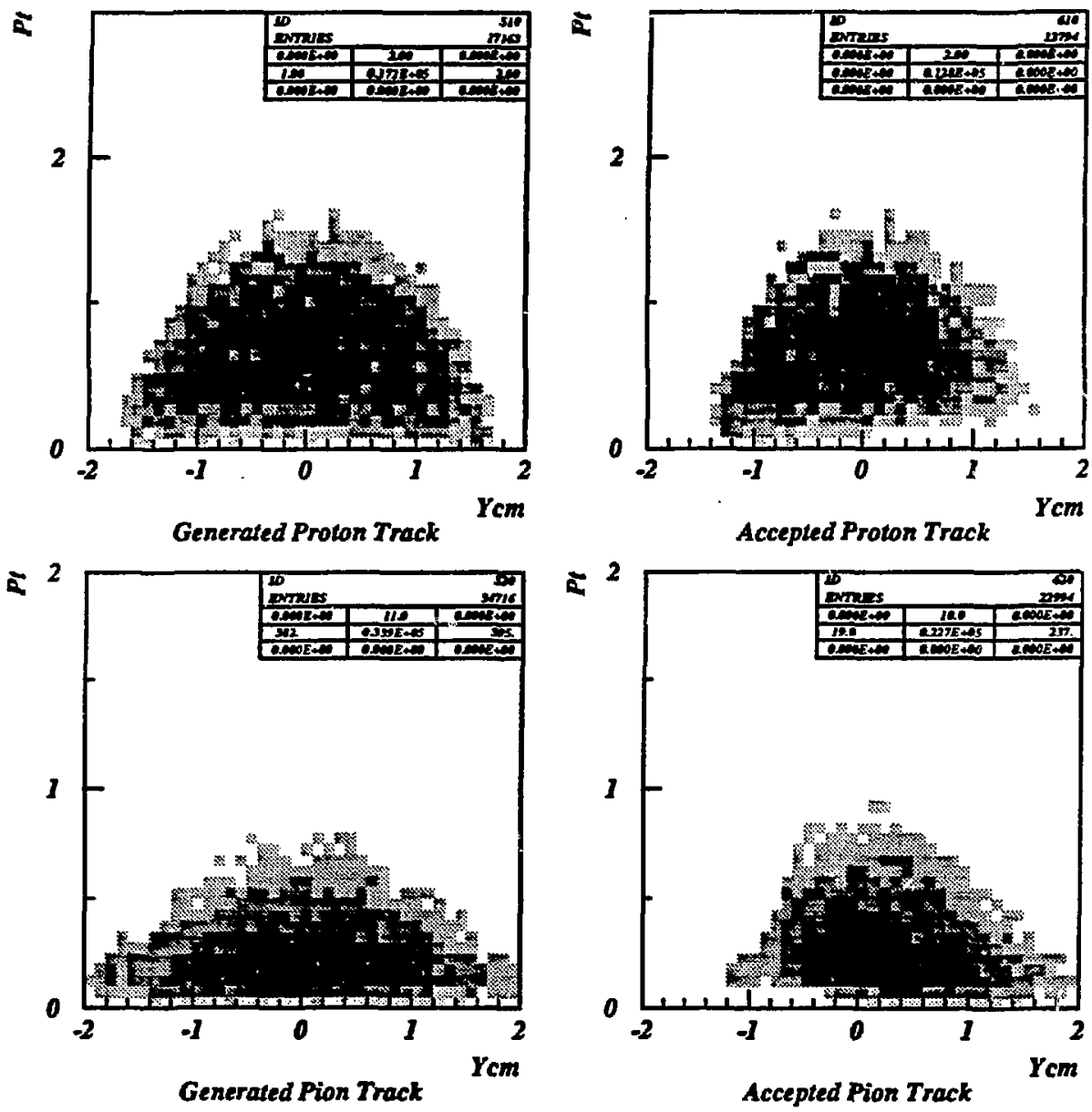

Figure 32: Transverse momentum vs rapidity ior protons and pions for $10 \mathrm{~A} \mathrm{GeV} \mathrm{Au}+$ Au RQMD simulations. 


\section{$A u+A u$ at $10.7 \mathrm{GeV} R Q M D$}
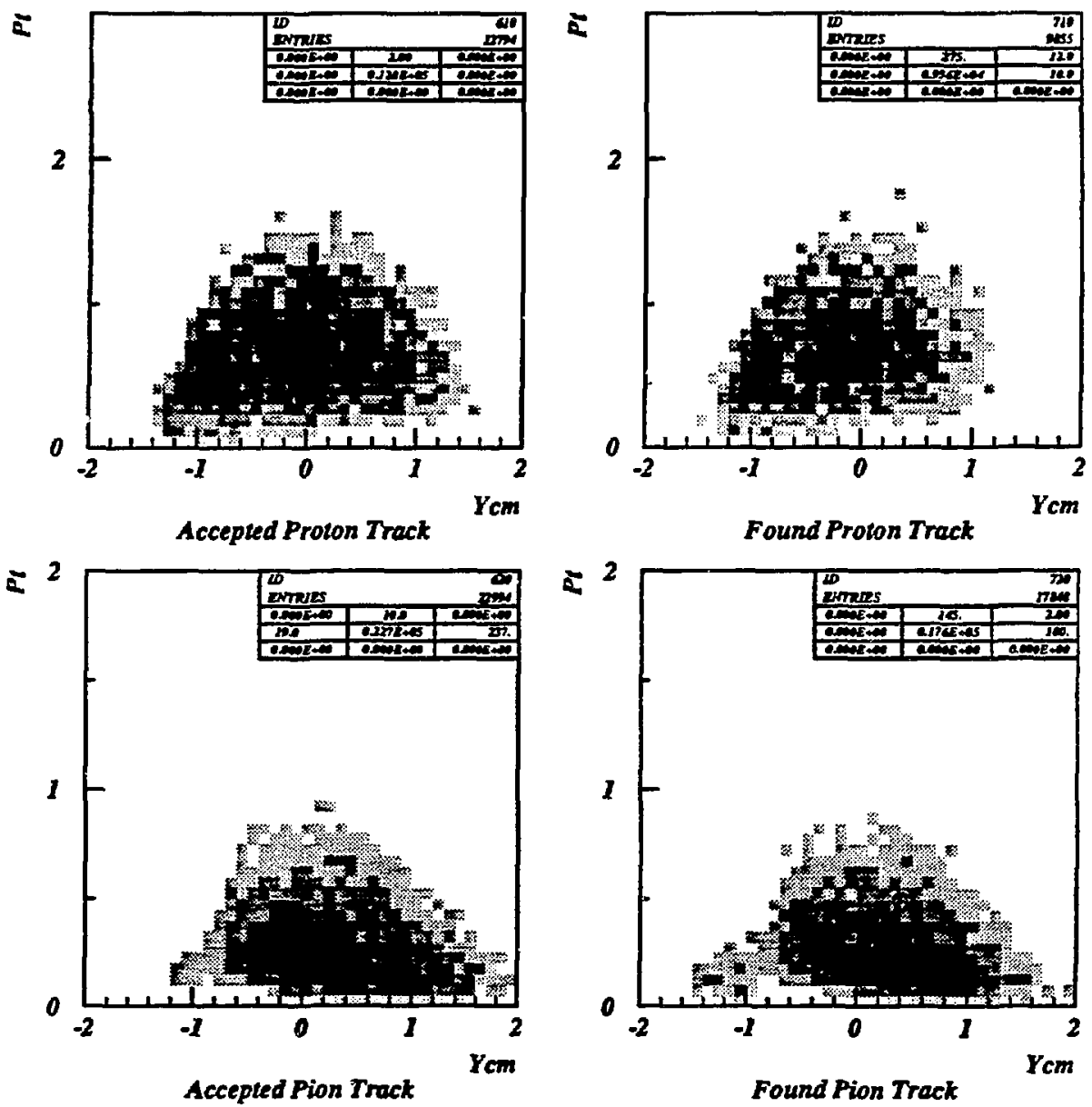

Found Proton Track

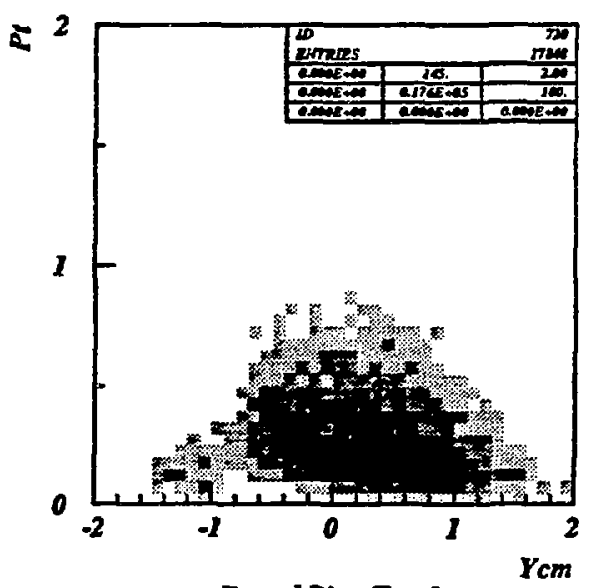

Found Pion Track

Figure 33: Transverse momentum vs rapidity for accepted protons and pions for $10 \mathrm{~A} \mathrm{GeV}$ $A u+A u R Q M D$ simulations. 


\subsubsection{Transverse Momentum Spectra}

The transverse momentum spectrum for protons is shown in Fig. 34 for Au + Au eveits at $6 \mathrm{~A} \mathrm{GeV}$ beam energy. The sections of plot correspond to "generated", "accepted" and "found" traclis at mid-rapidity. The fitted slope parameters (printed on the plot) are in good agreement with the generated events.

\subsection{The Multiple Sampling Ionization Chamber MUSIS}

Nuciear fragments heavier than $Z=7$ produce strongly jonizing tracks in the EOS TPC. Signals from these tracks exceed the dynamic range of the TPC electronics and consequently particle identification is lost. The MUSIC detector employs concepts from classical ionization chambers and TPCs. It provides unique charge identification and a precise determination of track position and angle for few particles in a wide mass range from Carbon to Uranium. The heavier projectile fragments $(Z>6)$ can be measured by placing the MUSIC detector downstream of the TPC.

An important goal at the AGS is to measure the momenta and $Z$ of all fragments as accurately as possible. By using reverse linematics, the projectile fragments are forward focussed and pass through the TPC and MUSIC detectors without loss of acceptance. Also because of jts outstanding charge resolution, the MUSIC detector allow events to be tagged according to secondary projectile fragments and correlated with information from the other detectors. For example, charge-changing cross-sections together with the loss of neutrons in a reaction could be studied.

\subsubsection{The MUSIC Detector}

The MUSIC Detector is shown in Fig. 35. It consists of three field cage structures which are aligned in a cylindrical vessel filled with an $\mathrm{Ar} \mathrm{CH} 4$ (P10) gas mixture at atmospheric pressure. The field cage have an active area of $100 \mathrm{~cm}$ by $60 \mathrm{~cm}$ and a depth of $48 \mathrm{~cm}$ each. The vessel (not shown) has an inner diameter of $124 \mathrm{~cm}$ and a length of $250 \mathrm{~cm}$. Two cages, (labeled 1 and 3 ) generate a horizontal drift field with a cathode plane in the middle and two anode planes on the left and right sides. The division into two halves increases the multiplicity capability in the horizontal plane. The central cage (labeled 2) generates a vertical drift field in which the cathode is at the top and the anodes are at the botton.

A heavy ion traversing the active gas volume creates an ionizing track of about $70 \times Z^{2}$ ion pairs per $\mathrm{cm}$. The unjform. field separates the electrons and positive jons. The electrons drift with constant velocity through the active volume and a grounded Frisch grid and arrive at the anode with a time delay proportional to their drift distance. The anode planes are subdivided aloing the main axis (i.e., along the path of the fragment) into 16 strips $3 \mathrm{~cm}$ wide. The signal from each strip is processed and digitized every $60 \mathrm{~ns}$ with a sampling ADC system. The distance from the anode plane is obtained from determining the centre-of-gravity of the signal in time. The track is reconstructed and identified from a total of 48 ( 32 horizontal and 16 vertical) projected positions and energy measuremtnts. 


\section{$A u+A u$ at $6 \mathrm{GeV} R Q M D$}
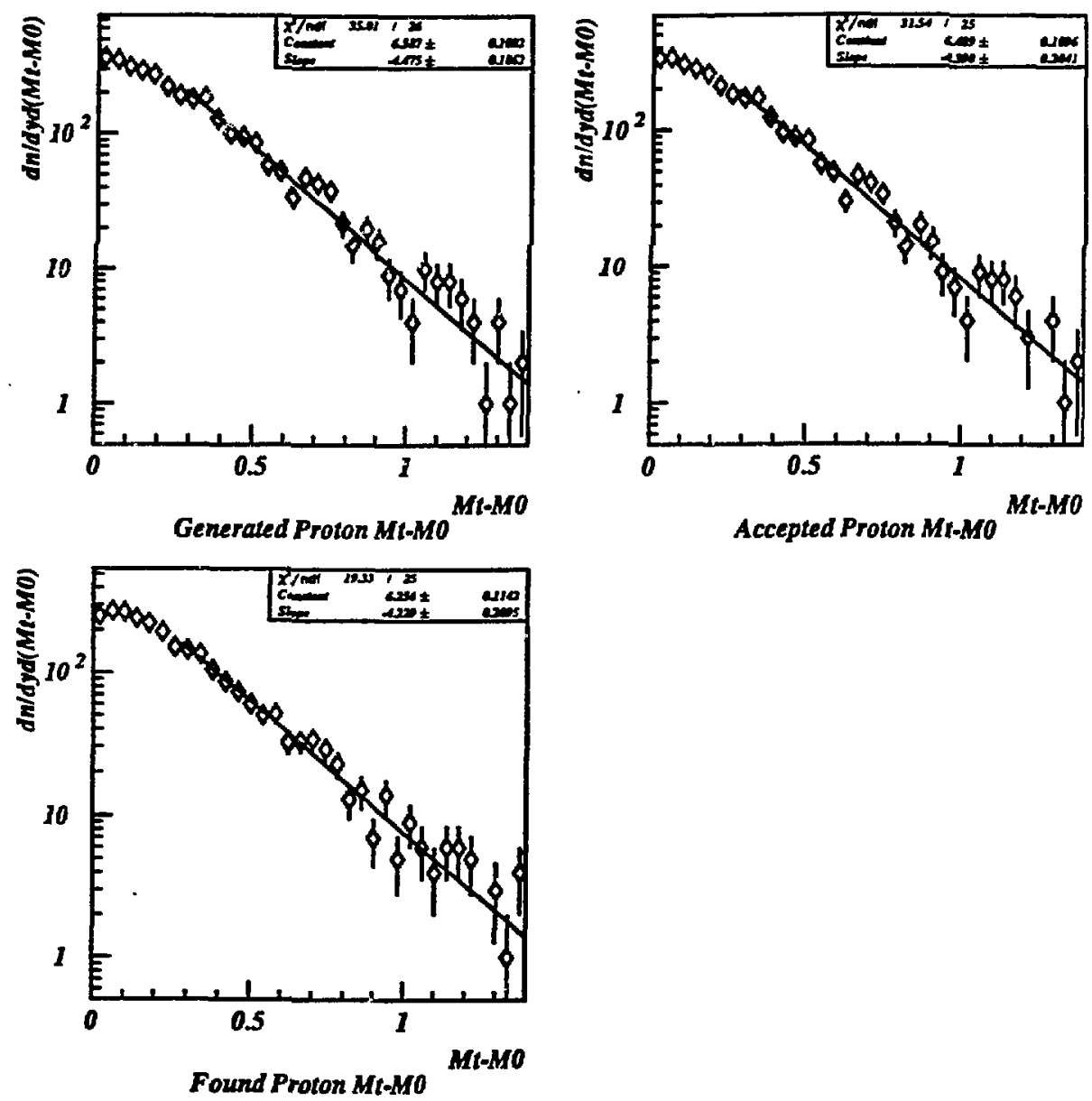

Figure 34: Transverse momentum spectra for mid-rapidity protons (generated, accepted, and found) for a $6 \mathrm{~A} \mathrm{GeV} \mathrm{Au} \mathrm{+} \mathrm{Au} \mathrm{RQMD} \mathrm{simulation.}$ 


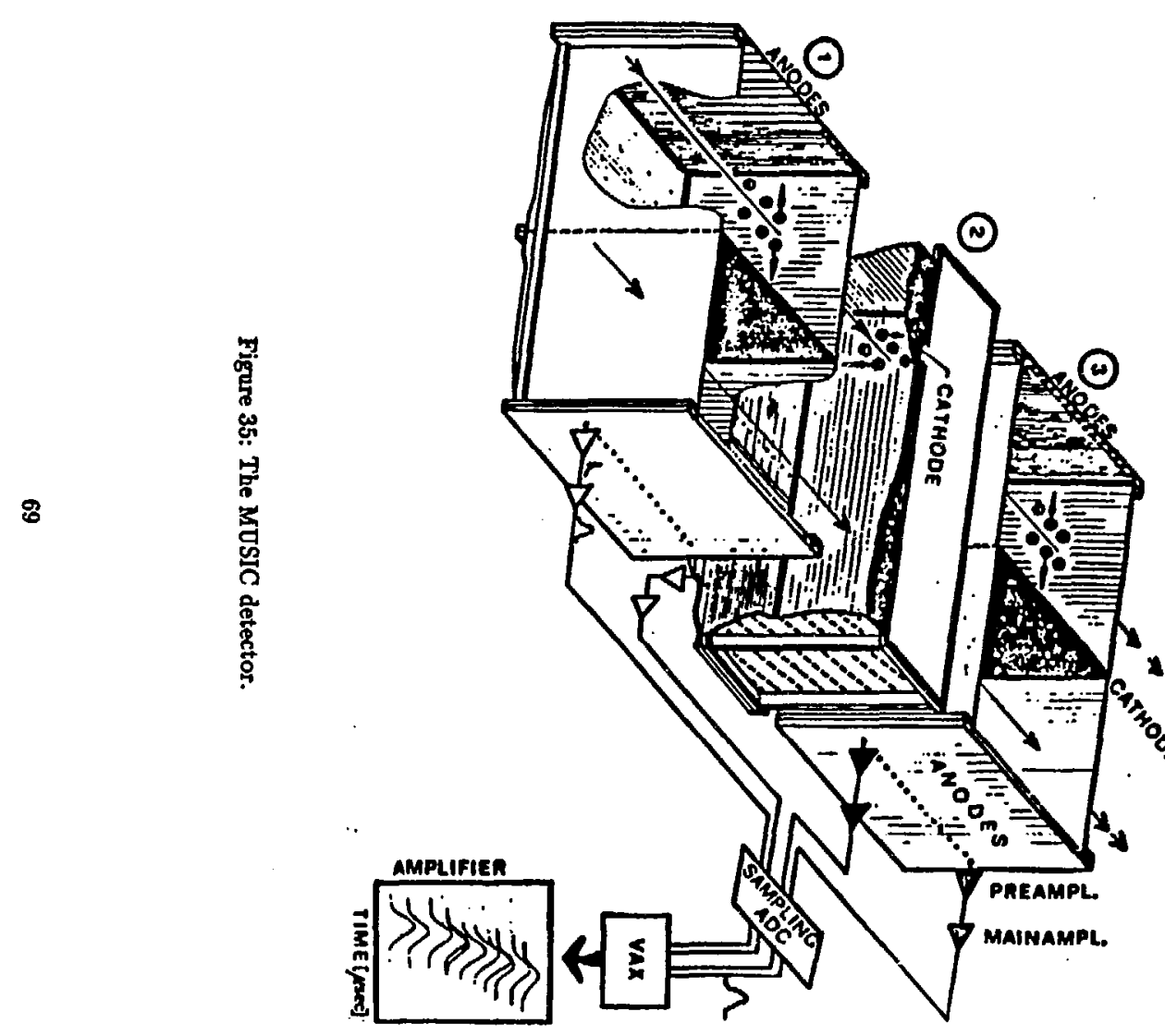




\subsubsection{Performance}

A reconstructed track in the MUSIC detector is shown in Fig. 36 . The charge of the fragment is determined as the square root of the sum amplitudes on all anode strips. It is not necessary to use truncated means because the distribution of energy deposition for a $3 \mathrm{~cm}$ gas layer is approximately gaussian for ions heavier than Carbon. The total charge resolution improves almost inversely proportional to the square root of the number of averaged anodes and $(n)$ can be parameterized by $\Delta Z=0.93 \times n^{-0.46}$.

The fragment charge spectrum for $A u$ beam interactions on a carbon target at $1.0 \mathrm{~A} \mathrm{GeV}$ is shown in Fig. 37. This histogram was obtained from experimental data taken at the Bevalac. Individual peals up to $Z=79$ are clearly resolved and are separated from each other by a single charge unit, i.e., the track average resolution is 0.35 units FWHM.

The position resolution of a single anode was determined from the fit residue for this anode after a track was fitted through all the other anodes. We obtain an rms resolution of $1.3 \mathrm{~mm}$ for $\mathrm{N}$ and $130 \mu \mathrm{m}$ for Fe. The position resolution is proportional to $1 / Z^{2}$ or to the noise-to-signal ratio:

$$
\sigma_{\text {pocition }} \approx \frac{V_{\text {drift }} \times t_{\text {rime }} \times \sigma_{\text {noine }}}{\text { signal }}
$$

The contribution of multiple scattering to the single anode position resolution is only on the order of $10 \mu \mathrm{m}$ and is negligible compared to the intrinsic detector resolution. The limiting factor in momentum resolution will be multiple scattering in the gas and materials of the EOS TPC, MUSIC, and the connecting space. For MUSIC, the angular resolution, $\sigma\left(\theta_{y}\right)$, is $\simeq 2 \mathrm{mr}$ at $400 \mathrm{MeV} / \mathrm{c}$ and $\simeq 1.5 \mathrm{mr}$ at $1 \mathrm{GeV} / \mathrm{c}$.

\subsection{The Time-of-Flight Wall}

The Time of Flight (TOF) Wall consists of $98,1 \mathrm{~m}$ by $2 \mathrm{~cm}$ by $7 \mathrm{~mm}$ slats of Bicron BC408 scintillating plastic. The slats are separated into two walls, the Small Wall Front (SWF) and the Small Wall Back (SWB). Each wall has 49 slats, so that the effective area of the TOF Wall is about one square meter. The walls are offset by $1 \mathrm{~cm}$, half a slat width, so that no fragments slip between adjacent slats. At each end of every slat, there is a one inch Hamamatsu photomultiplier tube (PMT) and a base which supplies the high voltage and splits the output signal. High voltage to the PMTs is provided by two LeCroy 1440 mainframes with plug in modules from the back. These units are controlled with a HyperCard stack running on a Macintosh.

One of the output signals enters a LeCroy 4413 leading edge discriminator which provides a stop signal, via ECL ribbon cable, to the LeCroy 2229 TDCs. The 2229 TDCs have been modified to give a dispersion of 30 ps per channel. The TDCs receive a common start from an upstream counter. The other signal from each PMT is sent to a splitter box via BNC cables. At the splitter box the signal is divided and one signal is atienuated by a factor of eight. Both of these signals are measured with a LeCroy 2280/2282A, 11 bit charge-sensing ADC system. The attenuated signal extends the dynamic range and therefore provides information on particles with charge $Z$ greater than 10.

Each scintillator slat has an input from a laser system for calibration. Light from the laser is dispersed and split into a single optic fiber per slat. At the present time a 


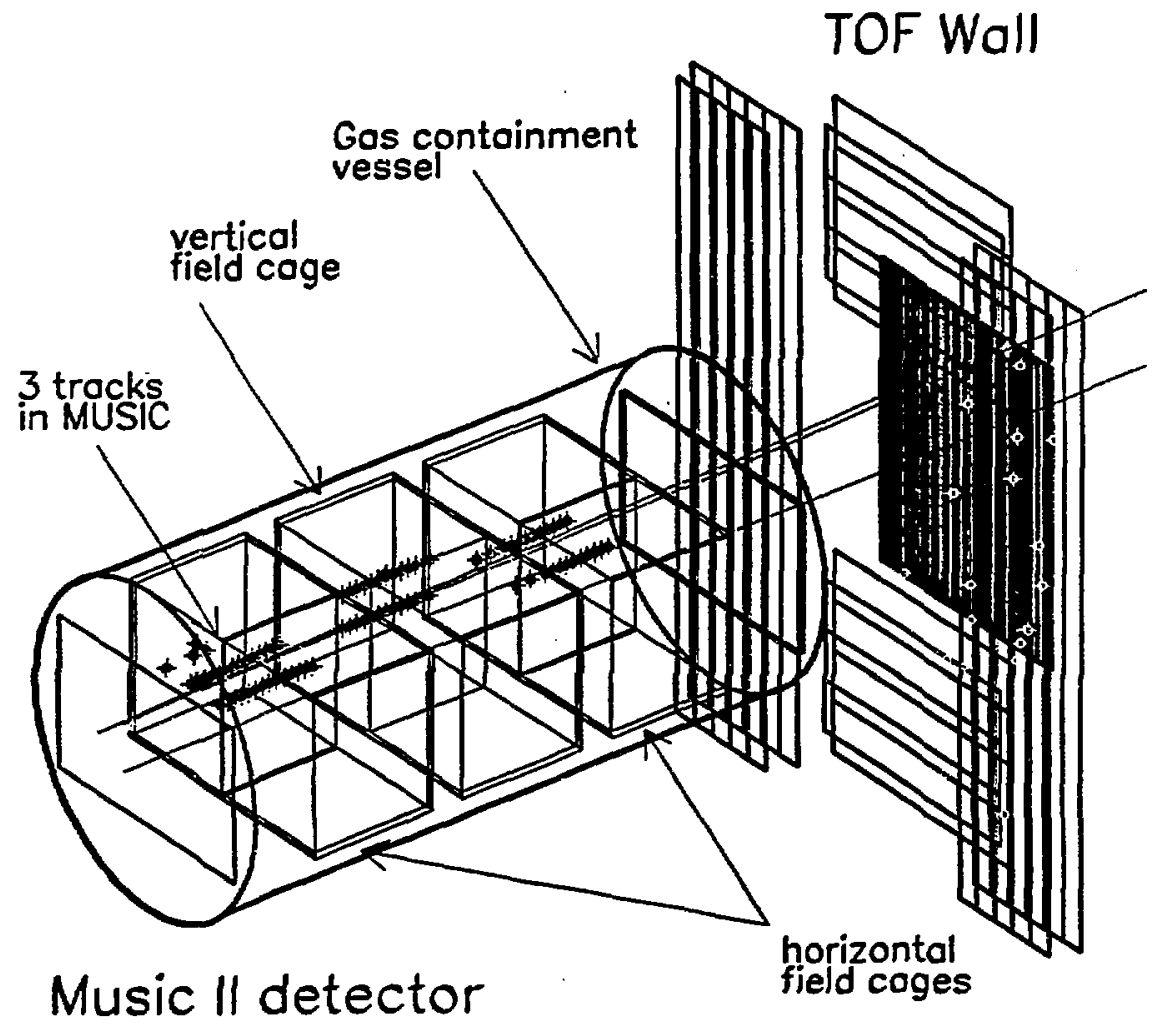

Figure 36: A track reconstructed in the MUSIC detector. Data from the Bevalac ron. 
Chorge Distribution in Music for L01000+C

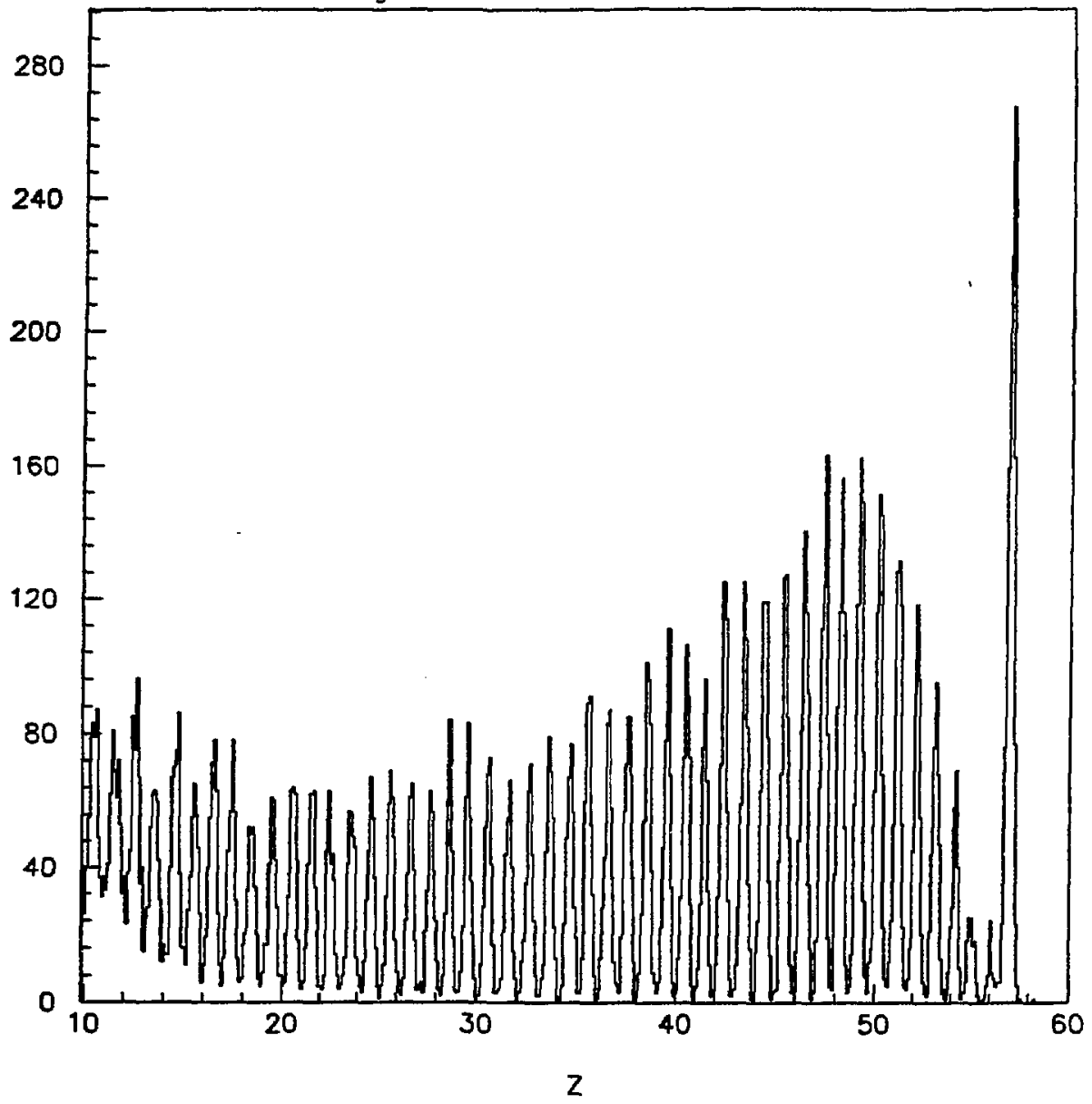

Figure 37: A charge spectrum as measured by MUSIC for $1.0 \mathrm{~A} \mathrm{GeV} \mathrm{Au} \mathrm{+} \mathrm{C} \mathrm{data} \mathrm{taken}$ at the Bevalac. 


\begin{tabular}{ll}
\hline$\sigma_{x}$ & $2.0 \mathrm{~cm}$ \\
$\sigma_{y}$ & $2.9 \mathrm{~cm}$ \\
$\sigma_{x}$ & $0.7 \mathrm{~cm}$ \\
Time resolution $\sigma_{t}$ & $240 \mathrm{ps}$ \\
Charge Resolution for $Z \geq 10$ & $0.4 \mathrm{e}$ \\
\hline
\end{tabular}

Table 6: Performance of the Time of Flight Wall.

calibration point is provide at one end of the slat.

A new laser timing calibration system is under consideration in which a pulse of light is transmitted to each PMT directly. This will allow walk and timing corrections to be made with the laser calibration data, rather than experimental data which is the method we used at the Bevalac. Briefly, we plan to fan-out a laser beam to individual PMTs. The fan-out unit consists of several shutters, splitters, a manual variable attenuator and a programmable variable attenuator.

Relative timing offset and charge calibrations were done with data from beam sweeps. Absolute timing calibrations and walk corrections are made with data and tracking information from other detectors. The overall performance of the TOF wall in the LBL EOS experiments is summarized in Table 6.

\subsection{The Neutron Spectrometer MUFFINS}

The modular MUlti-FunctIonal Neutron Spectrometer called MUFFINS, is designed to detect neutrons and measure their multiplicity, angular distribution and energy [63]. The main features of MUFFINS are position sensitivity, high efficiency and a small probability of detecting two neutrons in any individual element of the detector. The device consists of 30 thin discs of NE102A plastic scintillator. Six fast Hamamatsu R1398H photomultipliers (PMTs) are coupled to the disc edge by short cylindrical perspex light pipes arranged in a regular hexagon pattern. The face of each disc ( $1 \mathrm{~m}$ in diameter and $3 \mathrm{~cm}$ thick) is coated with reflective aluminized Mylar to optimize light collection and by absorptive black material around the edge. The 30 dises are mounted coaxially and stacked equally spaced $(6.5 \mathrm{~cm})$ from one another. In Fig. 38 we show the front view of the MUFFINS scintillator disc and its PMTs.

The signal from a PMT is split into two. One branch is directed into an ADC which measures the pulse height and the other is input to a fast leading edge discriminator, which in turn, is used to stop a common start TDC module. The START signal is taken from the experimental Trigger. The amplitude information is used to correct for slewing effects associated with the discriminators and for evaluating the multiplicity

A neutron impinging on the detector has a certain probability of passing through the scintillator discs before it interacts with one of them. When this happens a tiny flash of light is emitted which is seen and measured by all six PMTs belonging to that disc. The arrival times of light at the PMTs are measured with high accuracy $(\sigma \approx 100 \mathrm{ps})$ and used in an elaborate triangulation procedure to determine both the position and time of the neutron. In fact, only three PMT signals are necessary for hit reconstruction but the 


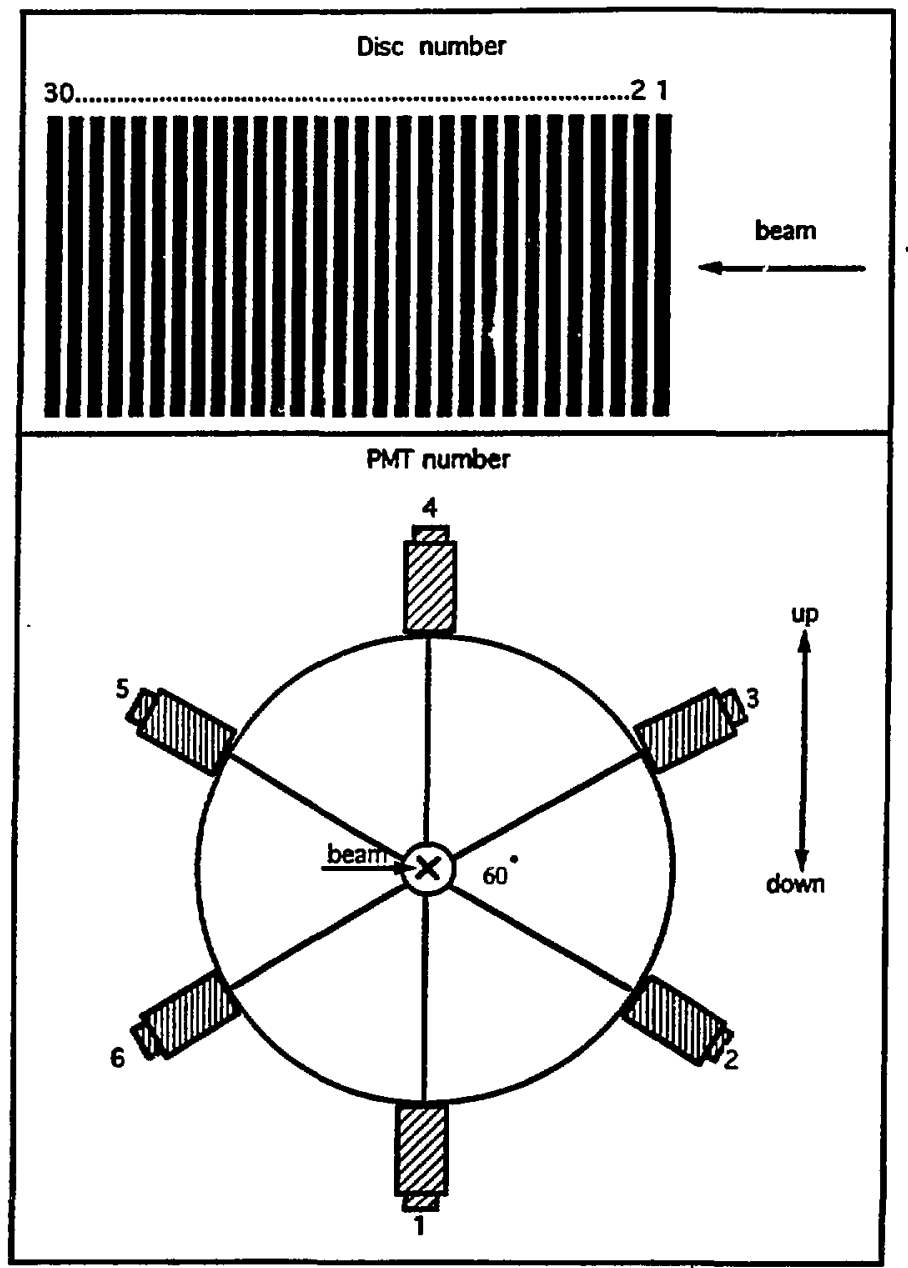

Figure 38: A front view of a MUFFIN disk and the PMTs. 
advantage of having redundancy in PMTs is threefold: First, infrequent double hits can be resolved. Second, the statistical uncertainty in the time-of-flight of the neutron from the target is significantly reduced by multiple measurement. The energy of the neutron is obtained directly from the known flight distance and the observed time-of-flight.

We have taken experimental data with MUFFINS and in a test run with ${ }^{32} \mathrm{~S}$ beam we have achieved a time resolution $\sigma \approx 55 \mathrm{ps}$ and a spatial resolution of $1.3 \mathrm{~cm}$. An example of the neutron energy spectrum measured with MUFFINS is shown in Fig. 39.

\subsection{1 n- $\gamma$ Discrimination}

At Bevalac energies, the neutrons arrive at the detector $\sim 100$ ps after the prompt $\gamma$ rays. Moreover, the energy deposited by a $\gamma$ ray in the scintillator disc is at most $1 / 7$ of that lost by a neutron. In MUFFINS, $n-\gamma$ discrimination is accomplished by setting the appropriate thresholds on early arrival times and the pulse beight amplitude. At the Bevalac we did not see $\gamma$ rays in the PMT time spectra since they where effectively cutout by the amplitude discriminator. The time resolution allowed us to separate up to $3 \mathrm{GeV}$ neutron from $\gamma$ rays at a distance of $5 \mathrm{~m}$ from the target.

We do not expect the situation to change significantly at the AGS energies, however simulations are in progress to check if the signals might appear above the discriminator thresholds. If so the distance from the target will have to be increased in order to get a better $n-\gamma$ separation in time.

\subsubsection{Energy Resolution}

The expression for the energy resolution $\sigma_{E} / E$ (where $E$ is the kinetic energy of the neutron) is derived in detail elsewhere[63]. Simple considerations show that both $\sigma_{E} / E$ and the angular acceptance $(\Delta \theta=R / d$, where $R$ is the radius of a disc) are inversely proportionaj to $d$ i.e., the distance from the target. Thus, better resolution is obtained at the expense of a smaller angular acceptance. For example, at $10 \mathrm{~m}$ from the target, and using a time resolution $\sigma_{t}=100 \mathrm{ps)}$ and a $\sigma_{d}=1 \mathrm{~cm}$, we obtain $\sigma_{E} / E=15 \%$ for $5 \mathrm{GeV}$ neutrons. However, for $1 \mathrm{GeV}$ neutrons (such as those emitted from a fireball in $5 \mathrm{~A} \mathrm{GeV}$ $\mathrm{Au}+\mathrm{Au}$ collision), we obtain $\sigma_{E} / E=1.8 \%$. In both cases the angle subtended at target is $5.6^{\circ}$.

\subsubsection{Detection Efficiency}

A neutrons is usually detected with small efficiency because it must first cause an interaction which yields a charged particle. The macroscopic cross-section for nuclear collisions is quite small in comparison to electromagnetic interactions. The scintillator disc thickness in MUFFINS was chosen such that each disc has a maximum intrinsic efficiency smaller than $4 \%$ (taking $\epsilon=N_{d}\left(\sigma_{C}+\sigma_{H}\right) t$, where $N_{d}$ is the number of CH "molecules" per cm ${ }^{2}, t$ is the thickness of thi disc and $\sigma_{i}$ the total cross section for producing charged particles by any process in neutron reactions with the $i$ th constituent of the molecule). This efficiency must then by multiplied by the joint probability of light reaching each PMT will produce a sufficiently high pulse to overcome the discriminator threshold. The low efficiency per 


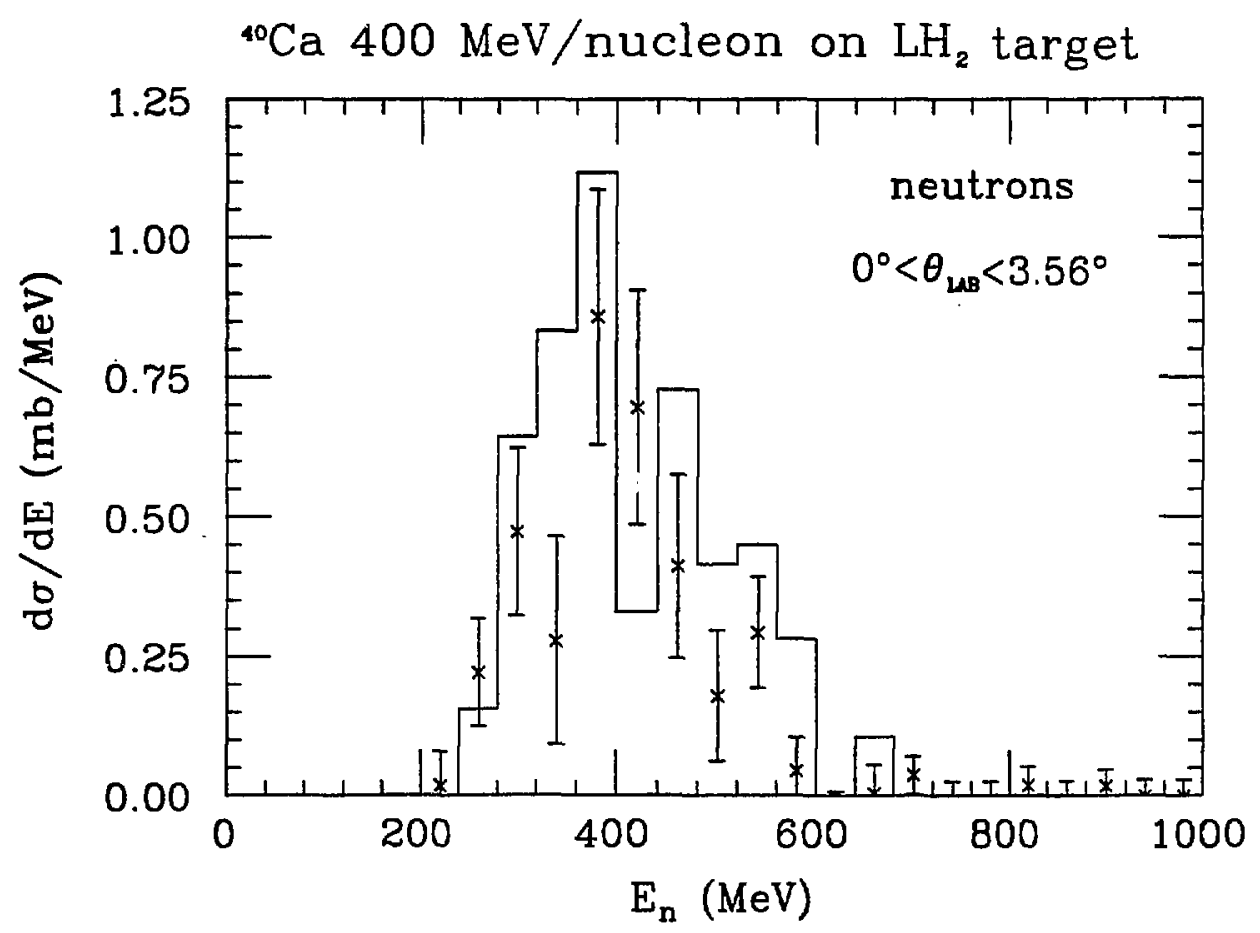

Figure 39: Energy spectrom measured with MUFFnNS for the ${ }^{10} \mathrm{Ca}+$ H reaction at $0.4 \mathrm{~A} \mathrm{GeV}$. 


\begin{tabular}{rrrrr}
\hline Theta & Geometric Acceptance $\epsilon_{g}$ & \multicolumn{1}{c}{ Total Acceptance $\epsilon_{t}$} \\
\hline & Projectile & Participant & Projectile & Participant \\
0 & $24 \%$ & $1.8 \%$ & $17 \%$ & $1.3 \%$ \\
15 & $5 \%$ & $1.5 \%$ & $3.5 \%$ & $1.2 \%$ \\
30 & & $1.1 \%$ & & $0.8 \%$ \\
\hline
\end{tabular}

Table 7: Efficiency of the MUFFINS for several angles.

disc insures that no more than one neutron is detected per disc in an event while keeping the intrinsic efficiency of the whole detector quite high due to the large number of discs.

Using GEANT, the efficiency for neutron detection of a single disc is found to vary slowly as a function of neutron energy ( $\simeq 2.25 \%$ at $200 \mathrm{MeV}, \simeq 3.5 \%$ at $1 \mathrm{GeV}, \simeq 4.2 \%$ at $5 \mathrm{GeV}$ and $\simeq 4 \%$ at $10 \mathrm{GeV}$ ). Thus the efficiency of the whole detector at AGS energies is close to $70 \%$.

\subsubsection{Geometric and Total Acceptance}

The geometric acceptance of MUFFINS is defined by the ratio

$$
\epsilon_{g}=\text { number of incident neutrons / total neutrons emitted from source }
$$

We have used a fireball model to separately characterize the distribution of projectile and participant neutrons. For a given fireball distribution (having an rms width $\sigma$ ), the geometric acceptance can be rewritten as

$$
\epsilon_{g}=\frac{2\left(\frac{R / d}{\sigma}\right)^{2}}{\exp \left(\frac{1}{2}\left(\frac{l}{\sigma}\right)^{2}\right)}
$$

provided $\frac{\Delta \theta}{\sigma}=\frac{R / d}{\sigma}<1$.

The total acceptance is the product of $\epsilon_{g}$ and the intrinsic efficiency of the whole spectrometer $(\approx 65-75 \%)$ i.e., $\epsilon_{t}=\epsilon_{g} \epsilon_{\text {intr }}$. The values of $\epsilon_{g}$ and $\epsilon_{t}$ are given in Table 7 as a function of angle $\theta$ measured with respert to the beam axis and assuming the detector is located $10 \mathrm{~m}$ downstream of the target.

We may decide to do separate measurements at $0^{\circ}$ and $30^{\circ}$ to isolate the projectile (cold) neutrons. Moreover, the energy resolution is also better for participant (fireball) neutrons than for projectile ones: thus for measurements at $30^{\circ}$ we could afford to place the detector closer to the target $(d=4 \mathrm{~m})$ and increase the acceptance for participant neutrons to $5 \%$. 


\begin{tabular}{lll}
\hline Beam Energy & $2-5 \mathrm{~A} \mathrm{GeV}$ & $5-10 \mathrm{~A} \mathrm{GeV}$ \\
\hline EOS TPC & $\begin{array}{l}\text { High tracking efficiency } \\
\text { Good particle ID }\end{array}$ & $\begin{array}{l}\text { Reduced tracking efficiency } \\
\text { Particle ID backward of } \\
\text { mid-rapidity at } 10 \mathrm{~A} \mathrm{GeV}\end{array}$ \\
MUSIC & $\begin{array}{l}\text { Good charge ID for heavy } \\
\text { mass fragments }\end{array}$ & $\begin{array}{l}\text { Good Charge ID for heavy } \\
\text { mass fragments }\end{array}$ \\
TOF & Good & $\begin{array}{l}\text { Not required for multi- } \\
\text { fragmentation studies. Will } \\
\text { be used to improve PID at } \\
\text { mid-rapidity. }\end{array}$ \\
MUFFINS & Good energy resolution & $\begin{array}{l}\text { Poor energy resolution at } \\
10 \mathrm{~A} \text { GeV beam energy }\end{array}$ \\
\hline
\end{tabular}

Table 8: Limitations of the Experimental Apparatus.

\subsection{Summary of Capability}

The performance of the experiment with regard to $\mathrm{Au}+\mathrm{Au}$ central collisions is summarized in Table 8. Generally spealing, all detector sub-systems work well in the $2-5 \mathrm{~A} \mathrm{GeV}$ energy range. At higher energies the EOS TPC has good performance (particle ID and tracking efficiency) backward of mid-rapidity which is adequate for studying collective flow effects in symmetric collisions.

It will not be possible to measure the neutron energy spectrum much beyond $7 \mathrm{~A} \mathrm{GeV}$ because of deteriorating energy resolution in MUFFINS. However, one can still make the angular and multiplicity distributions. Another option being explored, is to split MUFFINS into two stacks of 15 dises each, placed side by side. The detector would be located, space permitting, $20 \mathrm{~m}$ down stream from the target. In this way the energy resolution is improved while compensating for the loss of acceptance. A possible drawback is the reduction in neutron efficiency for each MUFFINS stack.

\subsection{Beamline Detectors and Trigger}

The trigger provides the data acquisition system with a valid START signal and is used to open the gating grid of the TPC. The trigger requirements are that it is made up quickly ( $<500 \mathrm{~ns}$ ) and selects target interactions ranging from peripheral to central collisions. The beam line detectors gererate the first level trigger. The detectors consist of two beam defining counters called S1V1 and S2V2, and an interaction counter referred to as ST. S1V1 and S2V2 are normal scintillator-veto doublets. One pair is placed before the last beam focusing element and the other is located before the target. The interaction counter is a simple large $(10 \mathrm{~cm} \times 10 \mathrm{~cm})$ area scintillator coupled to a R4332 Hamamatsu phototube capable of operating in a strong $(\sim 1.5 \mathrm{~T})$ magnetic field. The ST counter is 
mounted 2-3 cm after the target and is used as a veto counter. The reason for the small gap between the ST counter and target is to keep the number of beam-gas interactions after the target negligibly small. A high threshold setting on ST is ased to reject large amplitude signals which effectively removes beam particles, that is, a minimum bias trigger. The centrality of the collision is increased by lowering the discriminator threshold. The S1 scintillator also delivers to the MUFFINS and TOF detectors a start signal with optimum timing properties. A diagram of the beam line setup is shown in Fig 40. There are two additional detectors, called PLUTOs, which provide beam vectoring information. We intend to use them in our experiment if a comparable beam diagnostic is not available at the AGS. 


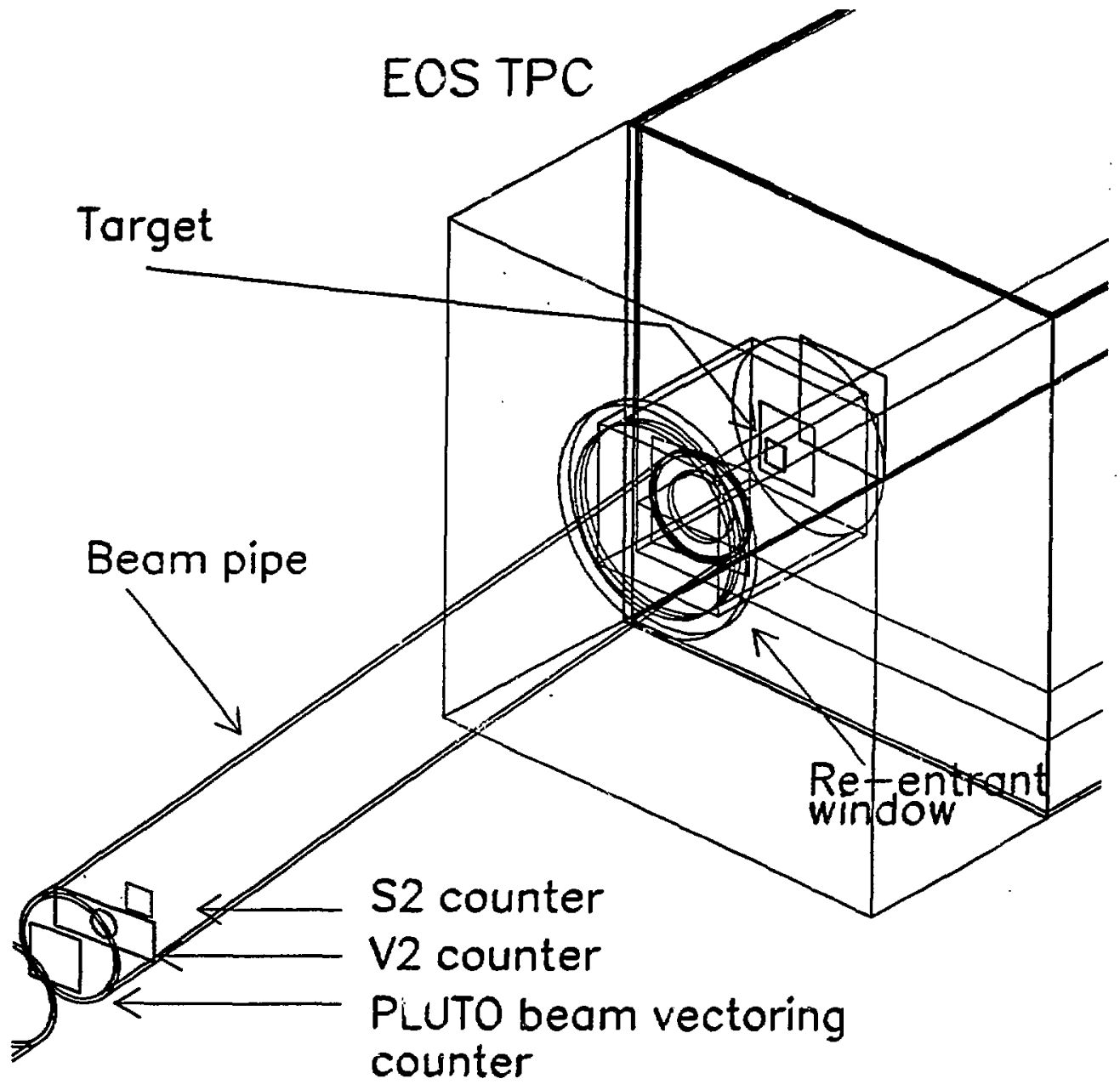

Figure 40: The beam line counter set $\mathrm{up}$. 


\subsection{Advanced Detector Technology}

In this section we outline two advanced detector concepts based on micro-fabrication technology. We intend to use these high performance devices if working prototypes are ready in time for the experiment. The devices under consideration are the gas micro-strip readout TPC and the Silicon Drift Chambers.

\subsubsection{The Micro-strip Readout TPC}

Recent developments in Micro-strip Gas Counters (MSGC's) have provided a novel approach to high resolution tracking in very high rate environment $[64,65,66,67,68,69,70]$. We are currently engaged in an R\&D program supported by the LBL Director's fund to investigate the capability of the Micro-strip Gas Counter. As a practical application, we intend to construct a small high-resolution Time Projection Chamber read out by a Micro-strip Gas Counter pad plane. This TPC will serve as a developmental prototype for the proposed AGS experiment and for large solid angle detectors envisaged for future experiments at RHIC and the LHC. We intend to study the performance of this new device with respect to tracking, accuracy, two track resolution and $d E / d x$ measurements in a high multiplicity environment - with and without the presence of a magnetic field.

The Micro-strip Gas Counter is built with thin metal strips on an insulating wubstrate by usin, the microfabrication technique. Figure 41 shows a typical structure of the Microstrip Gas Counter. It has $10 \mu \mathrm{m}$ wide amplifying anode strips and $90 \mu \mathrm{m}$ wide cathode strips and $200 \mu \mathrm{m}$ wide pitch. The metal strips are supported by electronic conducting glass and lept $10 \mathrm{~mm}$ away from the drift-plane. A back-plane can also be patîerned to obtain 2D information.

We have set up a test bed facility which includes a test chamber, gas system, electronics, and a PC based data acquisition system. We have fabricated several micro-strip samples at the Berkeley Microfabrication Laboratory at UC Berkeley. The micro-strip samples were tested in the $\mathrm{P} 10$ ( $\left.\mathrm{Ar} \mathrm{CH}_{4}\right)$ gas environment using the ${ }^{55} \mathrm{Fe} \mathrm{X}$-ray source. The $5.9 \mathrm{keV}$ photopeak and $3 \mathrm{keV}$ escape-peak were clearly visible with an energy resolution of about $18 \%$. Our effort is under way to improve the energy resolution, evaluate the rate capability, and optimize the design and processing to alleviate the high voltage discharge.

Figure 42 shows the schematic structure of the small TPC module which has a drift distance of $7 \mathrm{~cm}$, a width of $10 \mathrm{~cm}$ and a depth of $10 \mathrm{~cm}$. The final design will be optimized using GEANT simulations. Table 9 lists gas parameters such as diffusion constant, drift field and velocity, time bucket sampling for both P10 and DME gas.

The gas of choice seems is be DME rather than P10. DME gas has very low diffusion constants of $160 \mu \mathrm{m}$ which matches well to the MSGC readout when a pitch of $160 \mu \mathrm{m}$ is used. Comparing with P1n gas, DME gas has low drift velocity of $0.3 \mathrm{~cm} / \mu \mathrm{s}$ and requires a higher drift field. However, the major advantage of this TPC apart from its superior 8patial resolution is outstanding two track resolution listed in Table 9 under track area. For DME gas, two tracks are distinguishable if they are separated from each by $0.48 \mathrm{~mm}$ in any direction.

The use of DME gas also has less demand on electronics readout. A shaping time of $140 \mathrm{~ns}$ and clock frequency of $18 \mathrm{MHz}$ can be used. More time buckets are possible due 


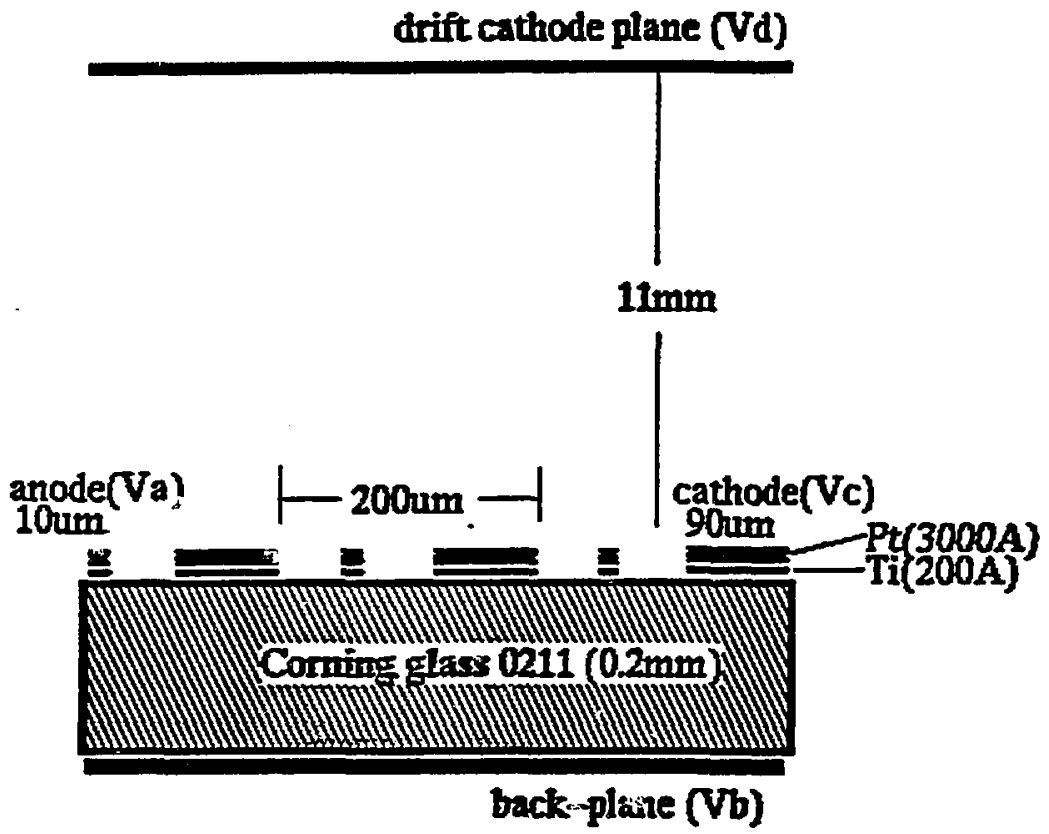

Figure 41: A Micro-strip gas counter. 


\section{A Sma11 TPC with MSGC Readout}

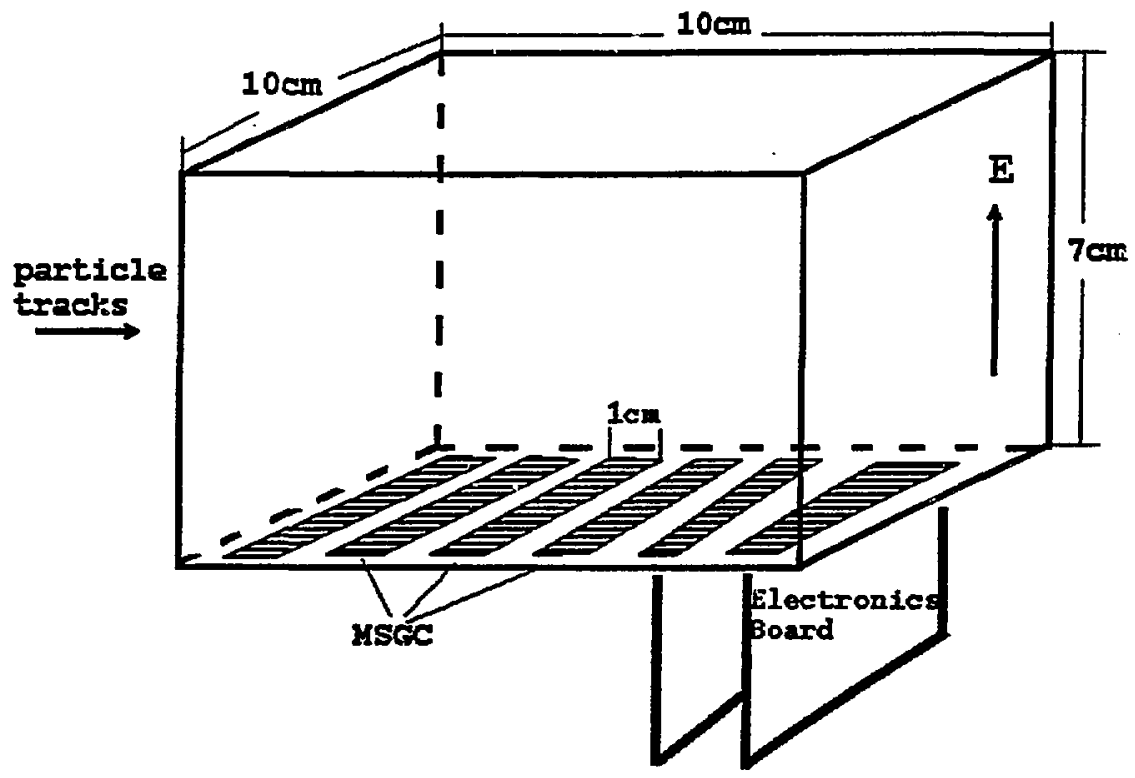

Figure 42: Possible design of a Micro-strip TPC. 


\begin{tabular}{lll}
\hline Parameter & P10 Gas & DME Gas \\
\hline$\sigma_{L}$ & $900 \mu \mathrm{m}$ & $160 \mu \mathrm{m}$ \\
$\sigma_{T}$ & $140 \mu \mathrm{m}$ & $160 \mu \mathrm{m}$ \\
$V_{\text {drift }}$ & $5.5 \mathrm{~cm} / \mu \mathrm{s}$ & $0.28 \mathrm{~cm} / \mu \mathrm{s}$ \\
Drift field & $130 \mathrm{~V} / \mathrm{cm}$ & $1000 \mathrm{~V} / \mathrm{cm}$ \\
Track area, $3 \sigma_{L} \times 3 \sigma_{T}$ & $2.7 \mathrm{~mm} \times 0.42 \mathrm{~mm}$ & $0.48 \mathrm{~mm} \times 0.48 \mathrm{~mm}$ \\
& $1.1 \mathrm{~mm}^{2}$ & $0.23 \mathrm{~mm}^{2}$ \\
Drift time & $1.3 \mu \mathrm{s}$ & $25 \mu \mathrm{s}$ \\
Shaping time FWHM & $38 \mathrm{~ns}$ & $140 \mathrm{~ns}$ \\
Time bucket & $15 \mathrm{~ns}$ & $56 \mathrm{~ns}$ \\
Clock frequency & $66 \mathrm{MHz}$ & $18 \mathrm{MHz}$ \\
Number of time buckets & 87 & 450 \\
Number of anodes & 710 & 620 \\
\hline
\end{tabular}

Table 9: Gas and matched TPC parameters for $7 \mathrm{~cm}$ of drift.

to the large drift time of $25 \mu \mathrm{s}$.

We plan to place this small TPC after EOS TPC to cover the very forward angular range where better two track resolution will benefit the reverse kinematic experiments.

\subsubsection{Silicon Drift Detectors}

The Silicon Drift Chamber is a relatively new type of semiconductor detector able to provide accurate position and ionization measurements with a modest number of readout channels. The detector is made from a very thin, rectangular, Silicon wafer. The silicon is fully depleted of mobile electrons. An ionizing particle passing through the wafer will create free electrons at the point of entry. An electrostatic field parallel to the surface is applied in order to transport the ionization electrons toward a collecting anode located at one end of the device. One coordinate is determined by measuring the time it takes the the electrons to reach the anode. The rms width of a charge cloud due to diffusion is quite large ( $150 \mu \mathrm{m}$ for a drift time of about $1 \mu \mathrm{s}$.) The anode is finely segmented into "pads" along the width of the detector, and thus electrons arriving at the anode are spread among several pads. This allows the other two coordinates of the incident particle in the plane of the detector to be determined by charge division readout. The position resolution is a few $\mu \mathrm{m}$ for drift distances up to several $\mathrm{mm}$ [71].

Currently, these detectors are being fabricated in p-type silicon at LBL as part of our effort to build a vertex detector for the STAR project. We have manufactured and tested a few n-type devices and recently obtained excellent results. A prototype STAR detector could be used in this proposal to gain experience in operating these devices in an accelerator environment and to contribute to the physics program outlined in Section 3.4. 


\begin{tabular}{|c|c|c|c|}
\hline Institution & Name & Status & Effort (FTE) \\
\hline \multirow[t]{6}{*}{ Lawrence Berkeley Laboratory: } & G. Rai & Staff Scientist & 0.5 \\
\hline & W. Gong & Post Doc. & 0.5 \\
\hline & L. Heilbronn & Staff Scientist & 0.25 \\
\hline & J. Miller & Staff Scientist & 0.3 \\
\hline & H. Wieman & Staff Scientist & 0.1 \\
\hline & C. Zeittlin & Staff Scientist & 0.25 \\
\hline \multirow[t]{8}{*}{ Kent State University } & D. Keane & Assoc, Prof. & 0.2 \\
\hline & M. Justice & Post Doc. & 0.3 \\
\hline & D. Weerasundara & Post. Doc. & 0.5 \\
\hline & H. Liu & Graduate student & 1.0 \\
\hline & $A$ Sort & Ph.D on EOS at AGS & \\
\hline & & Graduate Student & 0.2 \\
\hline & Y. Shao & Graduate Student & 0.2 \\
\hline & & & \\
\hline \multirow{5}{*}{ Purdue University: } & A. Hirsch & Prof. & 0.3 \\
\hline & R. Scharenberg & Prof. & 0.3 \\
\hline & N. Porile & Prof. & 0.3 \\
\hline & B. Shrivastava & Staff Scientist & 0.5 \\
\hline & Expect & $\begin{array}{l}\text { Graduate Student } \\
\text { Ph.D on EOS at AGS }\end{array}$ & 1.0 \\
\hline \multirow{2}{*}{$\begin{array}{l}\text { University of Texas: } \\
\text { at Austin }\end{array}$} & S. Mordechaj & Staff Scientist & 0.1 \\
\hline & Expect & $\begin{array}{l}\text { Graduate Student } \\
\text { Ph.D on EOS at AGS }\end{array}$ & 1.0 \\
\hline
\end{tabular}

Table 10: Mappower available.

\section{AGS Requirements and Logistics}

\subsection{Readiness}

This experiment can be made fully operational in a relatively short period of time since all major detector systems exist and no large scale construction is needed. Our biggest effort will involve relocation and installation at BNL.

The EOS TPC is a compact detector which does not require structural disassembly and can be shipped as a single unit. Likewise, the gas handling and chilled water recirculator systems, power supply racks and the data acquisition hardware can be shipped intact.

\subsection{Responsibilities}

The manpower available to this experimental program is listed in Table 10. Most of the 
collaborators from Kent State, LBL, Purdue Univ., U.C. Davis and Texas A\&M Univ., were involved in the EOS experiments at LBL and have expertise in the TPC, MUSIC, TOF detectors as well as in the DAQS (Data Aquisition), Beam Line Counters, Trigger and Software. There is sufficient manpower to cover all detector elements.

The primary responsibility for installation of the TPC at BNL will be shared by G. Raj (LBL), D. Weerasundara, M. Justice, H. Liu and D. Keane (Kent State). A. Scott, Y. Shao and S. Wang (Kent State) have considerable experience of the EOS program at LBL. They will be available for packing at LBL and/or set-up and debugging at BNL.

The Purdue and Texas (Austin) groups will take charge of the MUSIC and TOF detectors and some of the Beam Line counters. The Purdue group constructed the existing Beam Line at the BEVALAC and installed various rcintillator detectors used in the trigger set-up.

The Italian faculty participants from INFN, Catania (S. Albergo, Z. Caccia, S. Costa, A. Insolia, R. Potenza, J. Romanski, G. V. Russo and C. Tuve) are entirely responsible for the MUFFINS neutron spectrometer.

W. Gong and $H$. Wieman (LBL) are entirely responsible for the development of the advanced micro-strip TPC which is currently supported by LBL Director's R\&D funds.

At least three graduate students will earn their Ph.D. degrees from this experiment.

This list reflects the initial manpower available which we expect to increase as the project advances. The other collaborators have various commitments that limit their participation to a smaller percentage of time.

\subsection{Beam Line and Experimental Area}

EOS Time Projection Chamber: The EOS TPC requires a dipole magnet with gocd field uniformity in a $200 \mathrm{~cm} \times 100 \mathrm{~cm} \times 100 \mathrm{~cm}$ volume (Length $\times$ Width $\times$ Height). The actual space occupied by the TPC detector is approximately $250 \mathrm{~cm} \times 200 \mathrm{~cm} \times$ $100 \mathrm{~cm}$. The magnet should be rated up to $10 \mathrm{KG}$ with a field map available. The MPS magnet would be our first choice and therefore the $A 3$ beam line is a natural site for this experiment. The fallback options are the $\mathrm{B} 1$ (test area) and $\mathrm{C} 5$ beam lines assuming these lines can transport the lower energy heavy ion beams and a suitable magnet can be found. It is very desirable to have the beam kept under vacuum up to the target to avoid a beam fragmentation background. We assume beam diagnostics will be provided by the AGS.

In addition to the Magnet, the EOS TPC requires floor space for services and equipment in the vicinity of the detector as given in Table 11.

In the counting house we require a total of 5 standard racks. These will contain the TPC DAQS and the electronics for the MUSIC, TOF and MUFFINS detectors. Several patch panels to the cave will be needed. It is assumed that a local area (dedicated) Ethernet is avaliable.

MUFFINS Neutron Spectrometer: The complete MUFFINS detector rests on a cart $2.40 \mathrm{~m} \times 1.35 \mathrm{~m} \times 3 \mathrm{~m}(\mathrm{~L} \times W \times H)$ and weighs about 3 tons. It includes a motorized lift table to adjust the detector height. High Voltage (AV) to the PMTs is supplied by a LeCroy 1440 System mounted directly on the cart and needs 208-240 V a.c. outlet in the 


\begin{tabular}{|c|c|c|}
\hline Equipment & $\begin{array}{l}\text { Floor Area } \\
\text { Length, Width, Height }\end{array}$ & AGS Services Needed \\
\hline $\begin{array}{l}\text { Four racks containing } 32 \\
\text { power supplies for detector } \\
\text { electronics }\end{array}$ & $85 \mathrm{~cm} \times 235 \mathrm{~cm} \times 260 \mathrm{~cm}$ & $110 \mathrm{~V}$ a.c. \\
\hline $\begin{array}{l}\text { Refrigerated water recirculator } \\
\text { NESLAB HX-200 + Pump }\end{array}$ & $150 \mathrm{~cm} \times 215 \mathrm{~cm} \times 260 \mathrm{~cm}$ & $\begin{array}{l}110 \mathrm{~V} \text { a.c. } \\
230 \mathrm{~V} \text { a.c., } 3 \text { Phase, } 20 \mathrm{~A} \\
\text { Low conductivity water }\end{array}$ \\
\hline TPC Gas Handling System & $80 \mathrm{~cm} \times 80 \mathrm{~cm} \times 200 \mathrm{~cm}$ & $\begin{array}{l}110 \mathrm{~V} \text { a.c. } \\
\text { Nitrogen \& P10 gas lines }\end{array}$ \\
\hline $\begin{array}{l}\text { TPC Gating Grid and } \\
\text { miscellaneous electronics }\end{array}$ & $80 \mathrm{~cm} \times 80 \mathrm{~cm} \times 260 \mathrm{~cm}$ & $110 \mathrm{~V}$ a.c. \\
\hline
\end{tabular}

Table 11: Services required.

AGS experimental cave. Clean power for 2 Camac crates, 1 NIM crate is also requested. Dirty power should be avaliable to operate the lift table.

If the beam height is less than 90 in above the cave floor, then some mechanical work to resize the cart will be necessary. These modifications will be made by the Catania group prior to shipment.

Communication with the counting house is done via 200 RG58 cables, 12 17-pair twist' $n$ 'flat cables, 2 30-pair twist'n'flat cables and 18 -pair $3 \mathrm{M}$ flat cable.

The HV Power Supply, Discriminators and all cables are included with the detector. TDCs, ADCs and miscellaneous triggering modules will be obtained from the equipment pool. At the Bevalac a LeCroy 2280 ADC system consisting of 4 modules, and a processor in a Camac crate was used. We do not expect any problems in obtaining these items. Also, 23 Lecroy 2229 TDCs and a second Camac crate is required and we expect to obtain these on loan from either LBL, INFN or BNL.

\subsection{Beam Energies, Particles and Time Request}

The be: $n$ time request (see Table 12) is based on scaling factors such as the multiplicity of events as a function of beam energy and our experience with the event through-put of the TPC data acquisition system. The beam intensity should be limited to 1000-2000 Au ions/second in order to prevent positive ion build-up in the TPC gas volime. The AGS duty cycle should be adjusted accordingly. For lower $\mathrm{Z}$ beams, a higher beam rate can be tolerated subject to having a reasonably small pileup in the TPC and dead-time limitations due to taping speed. 


\begin{tabular}{|c|c|c|c|c|c|}
\hline $\begin{array}{l}\text { Beam Energy } \\
\text { A GeV }\end{array}$ & Projectile & Target & Events & $\begin{array}{l}\text { Time } \\
\text { hours }\end{array}$ & $\begin{array}{l}\text { Total } \\
\text { hours }\end{array}$ \\
\hline 10.7 & $\overline{\mathrm{A} u}$ & $\begin{array}{l}\mathrm{Au} \\
\mathrm{Cu}\end{array}$ & $\begin{array}{l}500 \mathrm{~K} \\
250 \mathrm{~K}\end{array}$ & $\begin{array}{l}242 \\
96\end{array}$ & \\
\hline 8.0 & $\mathrm{Au}$ & $\begin{array}{l}\mathrm{Au} \\
\mathrm{Cu}\end{array}$ & $\begin{array}{l}500 \mathrm{~K} \\
250 \mathrm{~K}\end{array}$ & $\begin{array}{l}242 \\
96 \\
\end{array}$ & 336 \\
\hline 6.0 & $\mathrm{Au}$ & $\begin{array}{l}\mathrm{Au} \\
\mathrm{Cu} \\
\mathrm{P}\end{array}$ & $\begin{array}{l}500 \mathrm{~K} \\
250 \mathrm{~K} \\
250 \mathrm{~K}\end{array}$ & $\begin{array}{l}228 \\
76 \\
48 \\
\end{array}$ & 336 \\
\hline 4.0 & Au & $\begin{array}{l}\mathrm{Au} \\
\mathrm{Cu} \\
\mathrm{P}\end{array}$ & $\begin{array}{l}500 \mathrm{~K} \\
250 \mathrm{~K} \\
250 \mathrm{~K}\end{array}$ & $\begin{array}{l}216 \\
70 \\
48 \\
\end{array}$ & 352 \\
\hline 2.0 & Au & $\begin{array}{l}\mathrm{Au} \\
\mathrm{Cu} \\
\mathbf{P}\end{array}$ & $\begin{array}{l}500 \mathrm{~K} \\
250 \mathrm{~K} \\
2 \mathrm{~b} 0 \mathrm{~K}\end{array}$ & $\begin{array}{l}212 \\
70 \\
48\end{array}$ & 334 \\
\hline & & & & & 330 \\
\hline Total & & & & & 1688 \\
\hline
\end{tabular}

T:ble 12: Beam times and energies requested. 


\subsection{Schedule}

The packing and shipping process can start in January 1994. We expect all the major components could be on the AGS floor by March 1994 and we estimate the complete experiment can be ready for beam by December 1994. 


\section{References}

[1] Karl-Heinz Kampert, J. Phys. G 15, 691 (1989).

[2] J. Aichelin, Phys. Rep. 202, 233 (1991).

[3] G. F. Bertsch and S. Das Gupta, Phys. Reps 160, 189 (1988).

[4] W. Ehehalt, Phys. Rev. C47, R2467 (1993).

[5] G. E. Brown, Comments Nucl. Part. Phys. 19, 185 (1990).

[6] G. F. Bertsch et al., Nucl. Phys. A000, 000 (1990).

[7] C. Hartnacl, Ph.D. Thesis, Univ, of Franlfurt, 1992, GSI-Report-93-05.

[8] W. Scheid et al., Phys. Rev. Lett. 32, 741 (1974).

[9] P. Danielewicz and G. Odyniec, Phys. Lett. B157, 146 (1985).

[10] J: Jaenicke and J. Aichelin, Nucl. Phys. A547, 542 (1992).

[11] T. J. Schlagel, S. H. Kahana, and Y. Pang Brookhaven National Laboratory Report No. BNL-47608 (1992)

Sidney H. Kahana, Yang Pang and Thomas J. Schlagel Physics at the AGS with a Relativistic Cascade Contribution to HIPAGS'93, MIT, (Jan. 13-25 1933).

[12] D. Beavis et al., Phys. Rev. C45, 299 (1992).

[13] M. Vient, Ph.D. Thesis, U.C. Riverside, 1988.

[14] S. Nagamiya, Nucl. Phys. A554, 5c (1992).

[15] T. Alexopoulos et al., Phys. Rev. D48, 984 (1993).

[16] N. S. Amelin et al., Nucl. Phys. A554, 463c (1992).

[17] H. Sorge, H. Stocker, and W. Greiner Ans. Phys. 192, 966 (1989).

[18] R. Mattiello. Private communication.

[19] J. Gosset et al., Phys. Rev. Lett. 62, 1251 (1989).

[20] T. Abbott et al., Phys. Rev. Lett. 70, 1393 (1993).

[21] S. A. Bass et al., Phys. Lett. 302B, 381 (1993).

[22] H. Bossy et al., Phys. Rev. C47, 4 (1993).

[23] T. Abbott et al., Phys. Rev. Lett. 66, 1567 (1991).

[24] T. Abbott et al., Phys. Rev. Lett. 64, 847 (1990). 
[25] S. E. Eiseman et al., Phys. Lett. B297, 44 (1992).

[26] J. Rafelski and B. Muller, Phys. Rev. Lett. 48, 1066 (1982).

[27] R. Mattiello et al., Phys. Rev. Lett. 63, 1459 (1989).

[28] A. Saulys et al., Contribution to HIPAGS'93, MIT, Jan. 93.

[29] S. Schnetzer et al., Phys. Rev. Lett. 49, 989 (1982).

[30] J. Harris et al., Phys. Rev. Lett. 47, 229 (1981).

[31] S. S. Padula and M. Gyulassy, Phys. Lett. B217, 181 (1989).

[32] A. Jahns et al., Univ. Franlfurt, Preprint UFTP 329/1993.

[33] A. Jahns et al., Z. Phys. A341, 243 (1992).

[34] A. Jahns et al., Phys. Rev. Lett. 68, 2595 (1992).

[35] T. Abbott et al., Pbys. Lett. B271, 447 (1991).

[36] S. H. Kahana et al., Phys. Rev. C47, R1356 (1993).

[37] D. Boal, C. K. Gelble, B.K. Jennings, Rev. Mod. Phys. 62, 553 (1990).

[38] Meson Interferometry in Relativistic Heavy Ion Collisions, BNL-48880, Brookhaven National Lab., April 16-17, 1993.

[39] M. Gyulassy, preprint LBL-32051 (1992).

[40] C. Greiner et al., Phys. Rev. Lett. 58, 1825 (1987).

[41] T. Abbott et al., Phys. Rev. C44, 1611 (1991).

[42] G. Peilert, Proc. XXXI Int. Winter Meeting on Nucl. Physics, Bormio, Italy.

[43] J. E. Finn et al., Phys. Rev. Lett. 49, 1321 (1982).

[44] R. W. Minich et al., Phys. Rev. Lett. B118, 458 (1982).

[45] A. S. Hirsch et al., Phys. Rev. C29, 508 (1982).

[46] J. Cugnon, Nucl. Phys. A462, 751 (1987).

[47] Y. Yariv and Z. Fraenkel, Phys. Rev. C24, 488 (1981).

[48] T. C. Sangster et al., Phys. Lett. B188, 29 (1987).

[49] M. Mehi et al., Phys. Rev. Lett. F0, 1936 (1988).

[50] N. T. Porile et al., Phys. Rev. C39, 1914 (1989).

[51] D. E. Gross et al., Phys. Rev. Lett. 56, 1544 (1986). 
[52] K. Nakai et al., Phys. Lett. B121, 373 (1983).

[53] X. Campi, Phys. Lett. 124B, 8 (1984).

[54] X. Campi, J. Phys. A19, L917 (1986).

[55] W. Bauer et al, Phys. Lett. 150B, 53 (1985).

[56] W. Bauer, Phys. Rev. C38, 1297 (1988).

[57] D. Stauffer, Phys. Rep. 54, 1 (1979).

[58] S. Havlin and D. Ben-Avraham, Advances in Physics 36, 695 (1987),

[59] J. C. Guillou and S. Zim-Justin, Phys. Rev, B21, 3976 (1980).

[60] H. Eugen. Stanley, Phase Transitions and Critical Phenomena Oxford University Press, New Yorl, ISBN 0-19-505316-8.

[61] G. Rai et al., IEEE Trans. Nucl. Sci. 37, 56 (1990).

[62] S. Wang et al., Phys. Rev. C44, 1091 (1991).

[63] S. Albergo et al., Nucl. Inst. and Meth. A311, 280 (1992).

[64] A. Oed, Nucl. Inst. and Meth. A263, 351 (1983).

[65] F. Angelini et al., Nucl. Inst. and Meth. A283, 755 (1989).

[66] F. Angeiini et al., Nucl. Inst. and Meth. A283, 755 (1992).

[67] T. Nagae, Nucl. Inst. and Meth. A323, 236 (1992).

[68] R. Bouclier et al., Nucl. Inst. : ind Meth, A323 240 (1992).

[69] RD22 Collaboration, CERN/DF:DC 92-51.

[70] SDC Collaboration, Technical Jjesign Report SSCL-SR-1215.

[7!] P. Rehak, Nucl.Instr. and Meth. A235, 224(1985). 